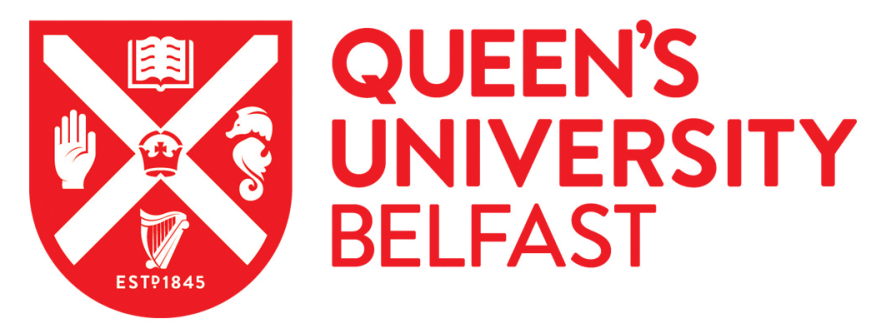

\title{
Microneedle array systems for long-acting drug delivery
}

Vora, L. K., Moffatt, K., Tekko, I. A., Paredes, A. J., Volpe-Zanutto, F., Mishra, D., Peng, K., Raj Singh Thakur, R., \& Donnelly, R. F. (2021). Microneedle array systems for long-acting drug delivery. European Journal of Pharmaceutics and Biopharmaceutics, 159, 44-76. https://doi.org/10.1016/j.ejpb.2020.12.006

\section{Published in:}

European Journal of Pharmaceutics and Biopharmaceutics

\section{Document Version:}

Peer reviewed version

\section{Queen's University Belfast - Research Portal:}

Link to publication record in Queen's University Belfast Research Portal

\section{Publisher rights}

Copyright 2021 Elsevier.

This manuscript is distributed under a Creative Commons Attribution-NonCommercial-NoDerivs License

(https://creativecommons.org/licenses/by-nc-nd/4.0/), which permits distribution and reproduction for non-commercial purposes, provided the author and source are cited.

\section{General rights}

Copyright for the publications made accessible via the Queen's University Belfast Research Portal is retained by the author(s) and / or other copyright owners and it is a condition of accessing these publications that users recognise and abide by the legal requirements associated with these rights.

Take down policy

The Research Portal is Queen's institutional repository that provides access to Queen's research output. Every effort has been made to ensure that content in the Research Portal does not infringe any person's rights, or applicable UK laws. If you discover content in the Research Portal that you believe breaches copyright or violates any law, please contact openaccess@qub.ac.uk. 
2 Lalit K. Vora ${ }^{1}$, Kurtis Moffatt ${ }^{1}$, Ismaiel A. Tekko ${ }^{1,2}$, Alejandro J. Paredes ${ }^{1}$, Fabiana Volpe3 Zanutto $^{1}$, Deepakkumar Mishra ${ }^{1}$, Ke Peng $^{l}$, Raghu Raj Singh Thakur ${ }^{1}$ and Ryan F. Donnelly ${ }^{1}$

$4 \quad$ ISchool of Pharmacy, Queen's University Belfast, 97 Lisburn Road, Belfast BT9 7BL, UK

$5 \quad *$ Corresponding Authors

$6 \quad{ }^{2}$ Department of Pharmaceutics and Pharmaceutical Technology, Faculty of Pharmacy, Aleppo

7 University, Syria

8

9 *Prof. Ryan F. Donnelly, Chair in Pharmaceutical Technology, School of Pharmacy

10 Queen's University Belfast, Medical Biology Centre, 97 Lisburn Road, Belfast BT9 7BL

11 UK

12 Tel: +44 (0) 2890972251

13 Fax: +44 (0) 2890247794

14 Email: r.donnelly@qub.ac.uk 
Abstract:

26

The development of microneedles (MNs) assisted drug delivery technologies have been highly active for more than two decades. The minimally invasive and self-administered $\mathrm{MN}$ technology bypasses many challenges associated with injectable drug delivery systems, by delivering the therapeutic materials directly into the dermal and ocular space and allowing the release of the active ingredient in a sustained or controlled manner. Different types of MNs (biodegradable solid/dissolving MNs and nanoparticle loaded/coated polymeric MNs or delivery by hollow MNs) have been envisioned for long-acting sustained delivery of therapeutic payloads, with the aim of reducing the side effects and administration frequency to improve the patient compliance. In this review, we covered the different types of MNs loaded with different nano/biotherapeutics for long-acting delivery for a wide range of potential clinical applications. We also outlined the future development scenario of such long-acting MN delivery systems for different disease conditions to achieve improved clinical benefit. Finally, we discussed the challenges lie ahead to realize the full potential of sustained-release long-acting MNs in the clinic.

Keywords: Microneedles; Long-acting therapy; sustained-release, controlled release, implantable delivery; nano-microparticles, intradermal delivery, site-specific delivery, transdermal delivery, Ocular delivery 


\section{Introduction}

In the field of drug delivery, the drug and device-based combined approach have improved the success rate in product development and clinical benefits for patients. Drug delivery through the skin, also called the transdermal route, is one of the most highly explored areas with this combined approach in research, due to several benefits over conventional oral and injectable drug delivery routes. Indeed, the transdermal drug delivery system (TDDS) has become an evolving area for systemic and local delivery of drugs, macromolecules and immunotherapeutics [1,2]. However, the poor permeability of therapeutics through the stratum corneum (SC) limits its application to only a selected few potent and highly lipophilic small molecules ( $\log$ p-value between 1 and 3, relatively low molecular weight <600Da). Passive permeation in this manner typically favours drug molecules with specific physicochemical characteristics. To deliver the wide range of low to high molecular weight drug and macromolecules, the $S C$ layer should be broken or punctured temporally by either physical or chemical method. One of such pioneering approaches is microneedles (MNs), expanding the possibilities of delivery to a wider range of drug molecules and biotherapeutics. Also, MNassisted drug delivery technologies have shown great potential to circumvent the invasive nature of conventional injections for greater patient acceptability and convenience. Furthermore, the recent research associated with MNs further extended the range of its use with a diversity of applications [2,3].

MNs typically consist of multiple microscopic projections of less than $2000 \mu \mathrm{m}$ in height, positioned on a single side of a supporting base or patch. MNs, also called microneedle (MN) arrays or microarrays patch (MAP) are used interchangeably in the literature. Upon application to the skin, the microprojections penetrate the epidermal layer and deliver or implant the compounds within the dermis below, wherein lies a high density of vascular and lymphatic beds available to absorb the drug systemically [4-6]. Besides the application of MNs for intradermal drug delivery, they have also been used for ocular drug delivery, as well as administrations of vaccines and DNA delivery [7-9]. MNs have been vastly exploited to enhance penetration of small and macromolecules through the skin or the ocular tissues in a pain-free manner with the potential capability of self-application. As our understanding of the biological skin/ocular barrier and MNs formulation aspects have improved over time, the design of controlled-release MNs based drug delivery systems have evolved from classical fast 
release mechanisms to the utilization of slow-releasing polymers or nano/micro-particles, further exploiting local biochemical changes to trigger and activate drug release [10,11]. Consequently, nanoparticle-loaded or slow-releasing biodegradable MN patches with prolonged therapeutic effect have been under investigation for long-acting self-administration potential for chronic conditions [12].

The general aim and scope of this review are to provide an informative account of long-acting sustained drug delivery possibilities with MNs that can be utilised for further development and clinical application. Initially, we introduced a general background on long-acting drug delivery systems and technologies. Then, we provided a detailed overview of MNs technology and the most utilized types of MNs employed in drug delivery. Following this, we offered a retrospective account of the up-to-date progression made in utilising the MNs in delivering long-acting formulations or sustaining the drug release as an alternative to the conventional hypodermic needles and, how ground-breaking work in this field has evolved to produce the current application of MNs-assisted delivery to long-acting formulations. In this review article, we discussed present progress and potential future use of MNs assisted long-acting drug technologies. Finally, we provided an expert opinion on the best selection of MNs technology for efficient delivery of long-acting drug formulations and discussed the future challenges and the translatable clinical potential of long-acting $\mathrm{MN}$ from bench to bedside.

\section{Long-acting drug delivery and technologies}

The prevalence of chronic diseases (e.g. type 2 diabetes, cardiovascular disease) is projected to worsen in the coming years. Chronic disorders require long-term medicinal therapy that affects the patient's day to day activities and substantially increases associated healthcare cost [13]. Conventional immediate-release drug delivery systems are subject to various limitations including poor patient compliance, dose missing or skipping, high levels of plasma fluctuations, multiple-dose drug therapy augments the risk of toxicity affecting patient compliance and adherence to the therapy and, the overall efficacy and cost of the treatment.

Long-acting drug delivery technologies based on sustained and controlled release mechanisms can be used to maintain constant plasma drug concentrations for extended periods as highlighted in the associated illustration (Figure 1). These have the potential to improve patient compliance compared to daily pills or injections [14] or may be employed to provide sitespecific drug delivery where the drug is most required and, thus, avoid higher systemic toxic exposure $[15,16]$. 
Indeed, long-acting dosage forms are mostly made of biodegradable and biodurable materials, thus, offer the potential to tailor drug release from hours to weeks or even to months for a wide variety of chronic disease conditions or prophylactic measures. Consequently, the wellestablished issues associated with conventional methods of delivery, such as those previously mentioned, can be easily overcome [17]. Furthermore, long-acting formulations could be particularly beneficial for classes of patients that are unable to adhere to treatment regimens, such as those suffering from psychiatric disorders [18].

Despite the substantial scientific output in this field, to date, no consensus in the terminology has been reached. A plethora of delivery technologies for long-acting formulations have been developed over the past few decades, however, inconsistent terms to describe the concept of long-term release are routinely employed: "long-acting injectable", "controlled release", "sustained release", "extended release" or, "depot" formulations [14]. The United States Pharmacopeia (USP) describes "extended release", a synonym for "prolonged" and, "sustained release", as "a deliberate modification to protract the release rate of an active pharmaceutical ingredient (API) in comparison to an immediate release dosage form" [19]. To date, it is still unclear whether even prolongation of a few hours could be considered as a sustained release or if only a protraction expressed in days is noteworthy. Although a handful of examples of long-acting formulations able to extend release only for few hours are mentioned in the present review, the majority of technologies we considered provide a sustained release of the API in a timespan of days at least.

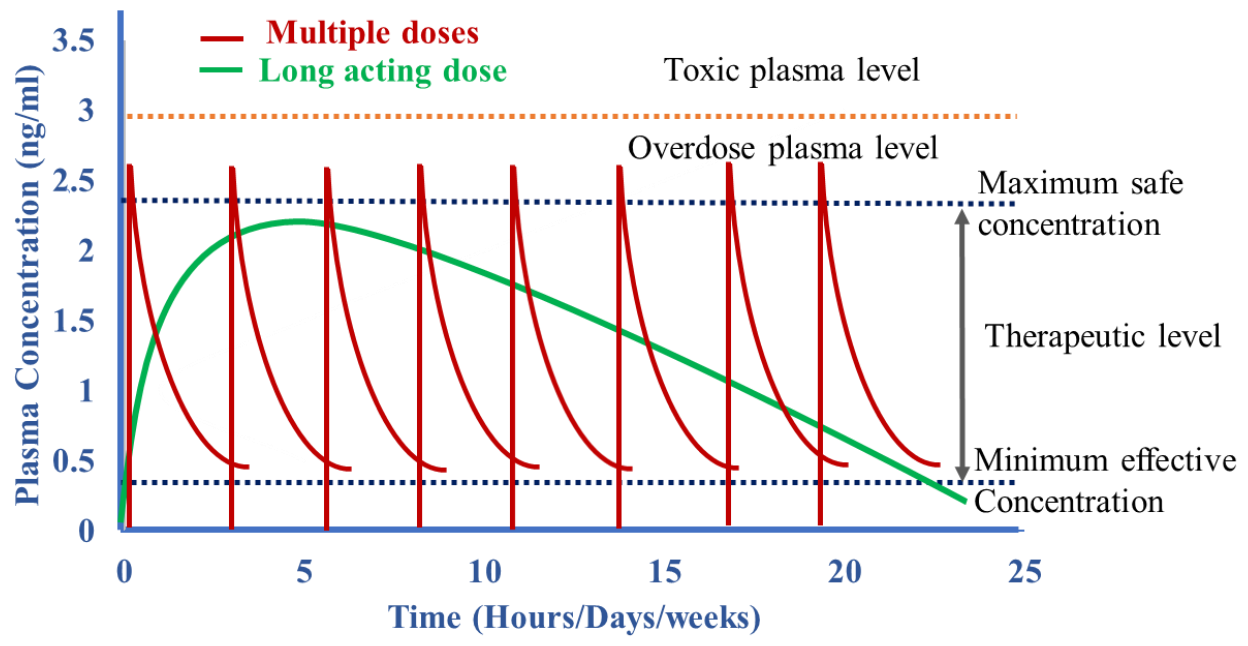

Figure 1. Representative pharmacokinetics of an ideal long-acting release formulation compared to the multipledose formulation. 
In the literature, an abundance of technologies has been used to produce long-acting formulations including, implantable devices, polymeric micro/nanoparticles, liposomes, nanocrystals and, thermo-responsive hydrogels [14,15,20-23]. A good example for implantable devices is the implantable pumps with glucose monitoring features used to deliver insulin in the management of diabetes [20] or, the intrathecal pumps to directly administer a drug to the spine for the maintenance of chronic pain [24]. These pumps are typically programmable "active" devices, which necessitate regular resupply of drug via an access port. Subcutaneous implants that are made of biodegradable polymers, provide long time, "passive" release without the need for replenishment is a further example. This kind of thin and flexible rod shape implant is particularly useful for highly potent drug delivery such as hormones [25].

Polymeric micro/nanoparticle-based injectable systems are another formulation strategy for long-lasting sustained-release. Microspheres $(1 \mu \mathrm{m}$ to $300 \mu \mathrm{m})$ and nanoparticles $(<1000 \mathrm{~nm}$ in size) are the first of such particle engineering-based approaches for sustained release injectables. Categories of such polymeric micro/nanoparticles may include polymeric micro/nanospheres and micro/nanocapsules. The former consists of polymeric particle matrices, whilst the latter consists of a matrix (generally oily) covered with polymers. Both types of particles can be produced using various biodegradable synthetic polymers such as polylactic acid (PLA) and poly(lactide-co-glycolide) acid (PLGA). in a broad range of sizes [23]. These vehicles have been used extensively as drug delivery systems in a wide variety of different locations in the body [23]. The first marketed microsphere-based product (Lupron ${ }^{\circledR}$ depot) was developed in 1985 by Takeda, for the palliative treatment of advanced prostatic cancer [26]. At present, 11 FDA-approved sustained-release microsphere injections are available on the market. These marketed microsphere products are mainly made from PLGA and PLA polymers. Microsphere products have additionally shown great advantages in comparison with conventional dosage forms. For example, Sandostatin Lar $^{\circledR}$ Depot resolved the short biological half-life of peptide drug $(\sim 2 \mathrm{~h})$, extending the dosing period to 4 weeks and, therefore, improving patient adherence [27]. Similarly, the bi-weekly injection of Risperdal Consta ${ }^{\circledR}$ (Risperidone) resulted in increased efficacy and fewer antipsychotic side effects than that of its conventional counterpart. Additionally, this conferred a further benefit due to the long-acting microspheres formulation, as patients experiencing psychosis no longer had to worry about remembering to take their medication every day [28].

Liposomes are another success story for sustained and targeted parenteral drug delivery. A liposome is an artificial membrane, which primarily consists of phospholipids and cholesterol 
$[29,30]$. The drug is then encapsulated within this phospholipid vesicle, in which the liposome then gradually degrades to release the drugs slowly. The liposome is the most versatile nanocarrier with superior biocompatibility and large-scale manufacturing possibility compared to other types of nanoparticles. The first FDA-approved liposome product, Doxil $^{\circledR}$ (doxorubicin (DOX) hydrochloride), reached market 1995 and is used to treat certain cancers. Following the success of Doxil ${ }^{\circledR}$, five more liposomal products for different disease conditions have emerged. Since the 1990s, drug nanocrystals or nanosuspension systems have attracted widespread attention, mainly because of the potentially sustained-release profile via the injectable route they afford. Abraxane ${ }^{\circledR}$, an albumin-bound nanoparticle formulation of paclitaxel, achieved great commercial success for the treatment of recurrent breast cancer [31]. The formulation of paclitaxel within Abraxane ${ }^{\circledR}$ has significant advantages over pure paclitaxel. At present, a total of 13 suspension and nanoparticle/nanocrystals injection products are now available in the market.

One of the key disadvantages of the above mentioned long-acting drug formulations, is that they typically necessitate hypodermic needle for delivery or perhaps surgical implantation of macroscopic devices within the body. In addition to being painful and causing stress, these invasive procedures generally are limited to use in hospitals or clinics and, thus, are not suitable for patient self-administration [32]. Subsequently, further development of a minimally invasive delivery system is now required that allows self-administration without causing pain to the patient. Rather than using a hypodermic needle to introduce advanced sustained-release formulations into the body, recent advances in MNs technology have given the functionality for both MNs and the long-acting formulations for controlled/sustained release of a wide range of drug molecules and vaccines.

\section{Microneedles}

MNs are becoming a more promising tool to bypass the physical barriers in the human body to deliver the therapeutic agents in a non-invasive manner. MN technology was first conceptualized for drug delivery to overcome the disadvantages and, preserve the benefits of hypodermic needles and traditional transdermal drug-delivery systems. The first compound delivered using MN was the low-molecular-weight dye, calcein [33]. Numerous studies quickly followed, leading to the present ever-growing body of proof for the significant drug delivery competencies of MNs [34]. Numerous MNs designs and types have been developed with the 
focus of achieving a pain-free, self-administrable device to replace the injectable hypodermic needles as described in the following section.

\subsection{Type of MN formulations}

208

209

210

211

212

213

214

215

216

217

218

219

220

221

222

MNs have been studied as an assisted drug diffusion device to deliver various types of therapeutics through the $S C$ to overcome the limitations of the conventional TDD approach [1]. To make the skin accessible to deliver or deposit wide range of drug and nanoparticles, various type of MN formulations (Figure 2) studied by manipulating MN characteristics (a type of MN, geometry, numbers of arrays per patch, drug formulation etc). These different MN characteristics can affect delivery mechanisms (bolus or sustained) in the dermis when applied [42]. A MN patch is made by assembling hundreds of MNs on a tiny baseplate to deliver enough drug to produce the required therapeutic effect. The amount of the drug that can be delivered is dependent on the properties of MNs including mechanical integrity, insertion capability and the loaded dose. Such properties are crucially affected by the geometry of the $\mathrm{MN}$ and, the number of MNs per patch. The therapeutic load delivery depends on the type of $\mathrm{MN}$ that directly placed in the epidermis or upper dermis layer which then reaches into the systemic circulation and shows a therapeutic response on reaching the site of action [100]. The mechanism of drug delivery through $\mathrm{MNs}$ is depicted in Figure 3. MN drug delivery technologies can be divided into several categories based upon their exact mechanism of delivery or, formulation: solid MNs for skin pre-treatment to increase the skin permeability, MNs coated with drugs, polymeric MNs that encapsulate therapeutic agents or particulate drug carriers and completely or partially dissolve in the skin, and hollow MN that permit drug infusion into the skin [60]. Recently, more categories of MN type considered such as separable $\mathrm{MN}$ and bioinspired MN.

\subsubsection{Solid MNs (Poke and Patch)}

Solid MNs are generally employed in the so-called 'poke with patch' approach to perforate the skin's $S C$ layer, creating transient aqueous microchannels that reach the deepest layers of the epidermis (Figure 2a) [42]. Following initial skin barrier disruption by MNs, a conventional transdermal patch, drug solution or gel is applied to the skin as an external drug reservoir for passive diffusion through these microchannels. The primary restriction of this methodology is the requirement for a two-step application process, which may lead to practicality issues for patients. Additionally, the micropores generated by MNs are quickly closed, resulting in reduced drug permeation. The water-insoluble and mechanically robust materials used to 
produce solid MN are classically silicon, metals, and polymers [43]. Disconcertingly, silicon and metals based MNs may remain solid after removal from a patient's skin, which may impose hazards of inappropriate re-use and, further lead to associated problems such as biofouling [44].

\subsubsection{Dissolving MNs (Poke and Release)}

241

The majority of recently published MN research focuses on dissolving MNs. This is owing to significant improvements in the drug loading in the polymeric MNs tips. Furthermore, dissolving $\mathrm{MN}$ arrays can now be produced from a wide range of biocompatible and biodegradable water-soluble materials, which is often achieved by relatively simple and inexpensive fabrication processes at ambient temperature, meaning they can be used to deliver a wide range of biotherapeutics. In contrast to coated MN, dissolving MNs have been created to entirely vanish in the skin without leaving any biohazardous sharps waste after use [35]. In this "poke and release" approach, the drugs can be loaded and released over time from the dissolving MN matrix (slow or rapid depending on the polymer composition) after skin insertion [36]. Dissolving MNs were designed to incorporate drugs or particles inside a watersoluble polymer matrix, and become fully dissolved once the MNs are implanted into the skin, with a dissolution time ranging from minutes to hours [37]. Dissolving $\mathrm{MN}$ have been previously fabricated to deposit the drugs into the skin for bolus and sustained release, thus allowing for the self-administration of therapeutics without the requirement of a hypodermic needle and syringe [35]. One of the most important features of dissolving $\mathrm{MN}$ is that the backing baseplate to which $\mathrm{MN}$ arrays are connected as a drug reservoir for sustained drug delivery throughout the application. This approach may increase total doses to milligrams the patch backing layer that swelled by imbibing interstitial fluid from the skin through channels created by $\mathrm{MN}$ arrays [35].

\subsubsection{Particle loaded MN (Poke, dissolve, and deposit)}

Particle integrated polymeric $\mathrm{MN}$ have been an emerging research topic, majorly due to advances in nanotechnology, thus potentially providing a wider therapeutic benefit $[38,39]$. Here, hydrophobic drug particles or slow-releasing drug-loaded particles (lipidic/polymeric) are localised in the MN matrix and, after the MN array is inserted into the skin, the MNs dissolve, thereby releasing the particles intradermally (Poke, dissolve and deposit) [15,39-41]. This particle-loaded polymeric MN approach has been shown to efficiently deliver both hydrophobic and hydrophilic drugs and expand the ranges of therapeutic agents [32, 35]. In recent days, this approach has become increasingly used in the deposition of nanoparticles 
within the skin, [42] as previously nanoparticles did not penetrate the skin's surface [44]. This approach allows the deposition of particles within the skin by one-step application with controlled/sustained release possibilities.

\subsubsection{Separable MN (Poke and deposit)}

273 Recently, in order to shorten the application times of the biodegradable or non-degradable MN,

274 fast separable tips from the baseplate have been designed (Poke and leave) (Figure 2d) [39,45].

275 This type of MN was fabricated by bilayer or multilayer techniques where a baseplate made of

276 fast dissolving materials or, a baseplate and MN tips separated by bubble structure or, fast-

277 dissolving layers (example, effervescent layer, freeze-dried porous layer) at the base of the

278 cone that rapidly separates the needles from the patch [45-48]. These are the most promising

279 tactic to implant the long-acting biodegradable MNs into intradermal or ocular space [49].

a)

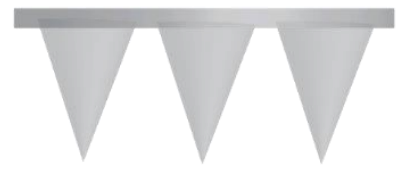

b)

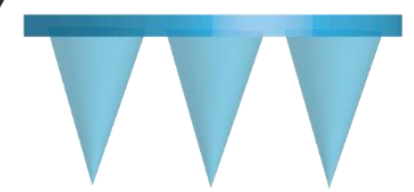

c)

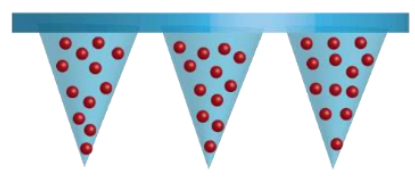

d)

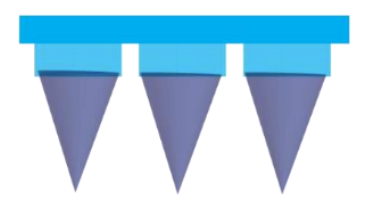

e)

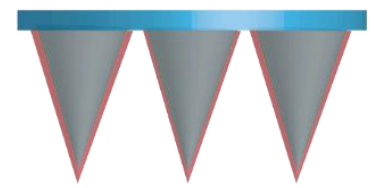

f)

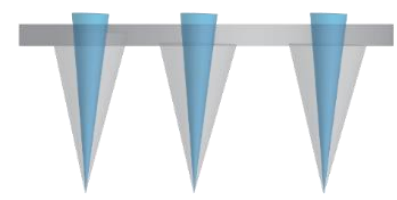

g)

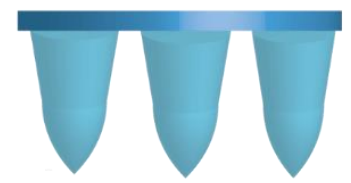

h)

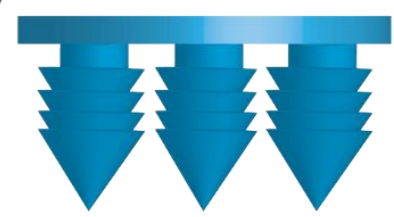

Figure 2. Different type of MNs. A schematic representation of six different MN types used to facilitate intradermal drug delivery (a) Solid MNs (b) Dissolvable MNs (c) Nano/microparticles loaded dissolvable MNs (d) Bilayer separable MN (e) Drug/ nanoparticles coated solid MN (g) Hydrogel-forming MNs h) Bioinspired MN 
287

288

289

290

291

292

293

294

295

296

297

298

299

300

301

302

303

304

305

306

307

308

309

310

311

312

313

314

315

316

This type of MN is first prepared by coating the solid MN with potent drug or vaccine formulation. This "coat and poke" approach allows the formulation to dissolve and release the coated drug or therapeutic into the subcutaneous tissue after skin insertion. Coated MNs have been mainly explored for the rapid intradermal delivery of potent molecules, particularly, biotherapeutics including parathyroid hormone (PTH) [50], desmopressin [51], salmon calcitonin [52] and DNA/RNA [53] to the skin [54].

\subsubsection{Hollow MN (poke and flow)}

With the help of advances engineering techniques, hollow MNs were designed to deliver the drugs intradermally through a controlled pressure-driven flow [55]. This "poke and flow" hollow MN approach was devised to introduce a drug solution or particles into the skin or eye by micron-size needles in a pain-free manner (Figure $2 \mathrm{f}$ ). This type of $\mathrm{MN}$ permits continuous delivery of drugs across the skin or ocular space through the $\mathrm{MN}$ bore using different techniques, namely, pressure or diffusion or electrically driven flow. This MN system is possibly capable of delivering larger amounts of drug substances in comparison to solid, coated, and dissolving MNs [27]. However, a major constraint to their use is the potential blockage of the needle openings during the dermal tissue insertion and flow resistance, thus, reducing drug release [56]. Therefore, various geometries (in-plane, out-plane, side-opened, tip-opened) of MN designs were investigated to achieve an optimum good liquid flow pattern without clogging the needles following skin insertion. Hollow MNs are made from a range of materials, including silicon and metal, glass, polymers and, ceramic [55].

\subsubsection{Hydrogel-forming MNs (Poke, swell and release)}

Hydrogel-forming MNs are a new developed type of MNs, prepared from drug-free swellable hydrogel-forming polymers (Figure $2 \mathrm{~g}$ ). They were first described and patented by Donnelly et al. in 2007 and, have now progressed to the forefront of MNs research in recent years [8,36]. Unlike dissolving MNs, the hydrogel-forming MN system is integrated with a drug reservoir (in the form of a film or tablet) on top of the MNs baseplate, and drug delivery is not limited to that loaded in the MNs projections. Therefore, milligrams of the drug can be delivered from even a relatively small patch size [57]. After application of the integrated drug reservoir-MNs patch to the skin, the inserted needle tips rapidly imbibe interstitial fluid from the intradermal space and, then allow the drug to diffuse through the swollen microprojections. Furthermore, 
the interstitial fluid absorption rate and release kinetics of the drug from hydrogel-forming MNs can be controlled by varying the polymeric composition and swelling properties of the hydrogel-forming MNs. The striking feature of this type of MNs is that they are removed intact from the skin, leaving no measurable polymer residue behind. However, they are sufficiently softened to preclude reinsertion, thus further reducing the risk of transmission of infection. These systems are manufactured using aqueous blends of various polymeric materials including Gantrez ${ }^{\circledR}$ AN-139 (methyl vinyl ether and maleic anhydride), Gantrez ${ }^{\circledR}$ S-97 (methyl vinyl ether and maleic acid) and poly (vinyl alcohol) (PVA). Gantrez ${ }^{\circledR}$ - hydrogel-forming MNs are prepared by crosslinking (via esterification reaction) with other polyol polymers/surfactants such poly(ethylene glycol) [58], pectin, [59] Tween 85 [60] and, hyaluronic acid [61]. PVA has been also used to prepare hydrogel-forming MNs by physical crosslinking (multiple freezethaw cycles). Demir et al. cast a formulation (gelatin and PVA) into moulds and the system was then frozen at $-20^{\circ} \mathrm{C}$ for $12 \mathrm{~h}$, followed by thawing at $25^{\circ} \mathrm{C}$ for $12 \mathrm{~h}$ to form hydrogelforming MNs [62]. The primary limitation of this methodology is that it may be timeconsuming and tedious. Recently, Tekko et al. developed a novel hydrogel-forming MN using an aqueous blend of PVA and PVP crosslinked with citric acid (esterification reaction) at 130 ${ }^{\circ} \mathrm{C}$ in a short time (45 -180 minute) [63]. The new hydrogel-forming MNs exhibited sufficient mechanical properties to be inserted in the skin and moderate swelling in the range of 250$600 \%$. Furthermore, they possessed the ability to control the delivery rate (without affecting the drug delivery efficiency) by adjusting the polymeric composition and crosslinking time. This type of MN has been used to deliver a wide range of small hydrophilic and hydrophobic drugs and macromolecules in a sustained manner [63-66].

\subsubsection{Bioinspired MN (Easy to poke and hook)}

As the name suggests, this MN design is inspired by natural phenomena, where the different shape of sharp needle objects in insects or plants, used to suck the food or protect against the harm. The lateral faces of this biomimetic MN designs were ornamented with bug-inspired microstructures [67]. Extensive work is being carried out regarding an insight into female mosquitos' proboscis (mouthparts) insertion into the skin $[68,69]$. For the first time, Oka et al. manufactured a hollow silicon MNs with a jagged shape inspired by mosquito proboscis [70]. This type of biomimetic needle can significantly reduce the required insertion force (Easy to Poke). Additionally, MNs with swellable tips inspired by the proboscis of an endoparasite (Pomphorhynchus laevis) were proven to be efficient in the adhesion to and interlocking with the tissues (Hook) [71]. This biomimetic MN platform governed by shape change-mediated 
mechanically interlocking MNs. These bioinspired multifunctional MNs inherited their advantages such as easy insertion, strong adhesion, tissue interlocking and, thus, are postulated to permit broad applications to various conditions.

\subsection{Manufacturing of $M N$}

The first patent on MNs drug delivery device as 'puncturing projections' was filed in the US in the year 1971 by Gerstel and Place [72]. However, it took 20 years from initial conception to produce the first true MN-mediated delivery; silicon MNs that demonstrated the successful delivery of calcein across human skin. Since then, advancement in the microelectronics and microfabrication technologies has led to faster development of the different forms of $\mathrm{MN}$ from various non-biodegradable and degradable materials. MNs have been demonstrated to be efficient in the delivery of many therapeutic agents across biological barriers, including skin, sclera, and mucosal tissue. It has also been reported recently, that $\mathrm{MN}$ arrays can be utilised to not only transport small molecules but, can be used to transport larger molecules and even nano/microparticles [33,54,73].

Dissolving/polymeric MNs have also been reported and, are known to be made from many biocompatible materials such as sugars and polymers. The most commonly employed polymers that are used in the fabrication of MNs are; PLA, polyglycolic acid (PGA), PLGA, poly(vinyl alcohol) (PVA), poly(vinylpyrrolidone) (PVP) and carboxymethyl cellulose (CMC) [32,35,38]. Polymeric MN has gained research interest recently, because of their simple micromoulding fabrication technique, in which MNs are prepared using a polydimethylsiloxane (PDMS) mould. PDMS moulds are created as precise inverse-replicates of the master structures. These male master structures normally produced from metallic, polymeric or silicone materials that are obtained through techniques such as 3D-printing [74], lithography [75], etching [76], thermal drawing [77] and, laser micromachining [78]. Anisotropic etching of silicon wafer in $\mathrm{KOH}$ is a commonly used method for obtaining a sharp pyramidal structure that can be replicated by pouring elastomer, such as PDMS, which may then be further used as a production mould for obtaining a large number of MN patches [79]. Nowadays, simple and flexible manufacturing technique, two-photon polymerisation based 3D printing was employed to fabricate various size and shape of MN templates with high precision and low cost in a short time [74]. PDMS is a hydrophobic flexible material, which can be used to accurately reproduce the master structure as a negative template $[36,80]$. PDMS moulds are typically fabricated by pouring PDMS solution over the male master structure and allowing the 
polymer to cure at $60-80^{\circ} \mathrm{C}$ for less than $6 \mathrm{~h}$ or overnight at room temperature, depending on

383

384

385

386

387

388

389

390

391

392

393

394

395

396

397

398

399

400

401

402

403

404

405 the specific protocol provided by PDMS supplier [74,81]. Another advantage of using PDMS moulds is that the master template can be re-used for MN fabrications after thorough cleaning of the mould's cavities. The principal benefit is the low cost of polymeric materials and their relatively simple fabrication by micro-moulding processes at ambient temperatures, which should allow for straightforward scale-up to industrial mass production [36].

Another type, Coated MN, has been relatively well documented in the literature or the delivery of potent compounds and vaccines [82]. Various fabrication techniques have been utilized to efficiently coat the individual MN for the fabrication of metal or polymer-based coated MNs including dip coating [83] spraying [84], gas-jet drying [85], and inkjet printing [86,87] and electrohydrodynamic atomization (EHDA) [88]. However, inconsistency arises in the uniformity and accuracy of the dosage coating of MNs, which may overcome by controlling coating formulations such as solution viscosity and contact angle [89,90]. Layer-by-layer coating techniques have also used for temperature-sensitive molecules such as DNA and proteins. This is achieved by coating the negatively charged proteins or DNA molecules onto the metal or polymer base mould and, the cationic charged polymer solution was, in turn, dipped to form a polyelectrolyte multilayer [91].

The manufacturing methods for hollow MNs utilised the wide range of fabrication technique include MEMS [92], lithography methods [93], laser cutting [94], laser ablation, metal electroplating[95] isotropic and anisotropic etching [96], injection moulding, surface/bulk micromachining, polysilicon micromolding, and additive manufacturing (AM) technologies [97], that more commonly known as 3D printing such as stereolithography (SLA) [98] and two-photon polymerisation [74]. 

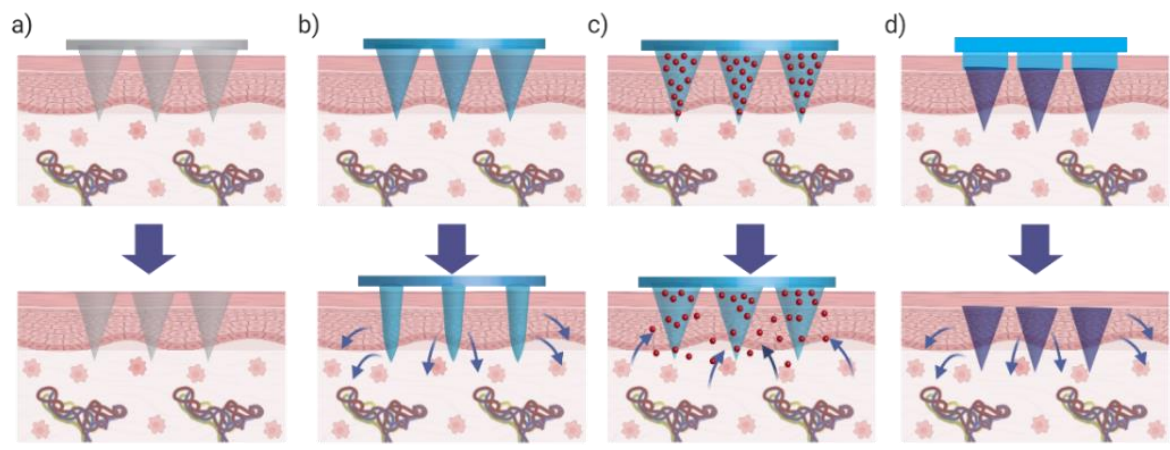

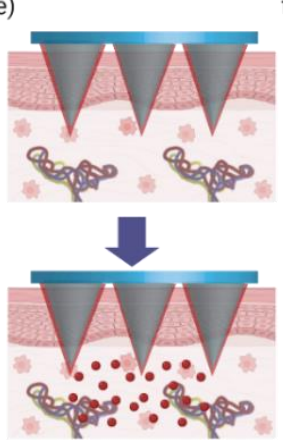

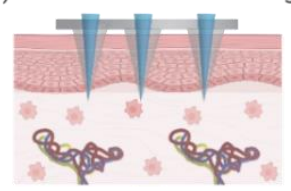

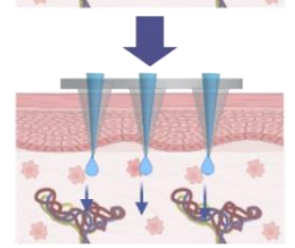

g)

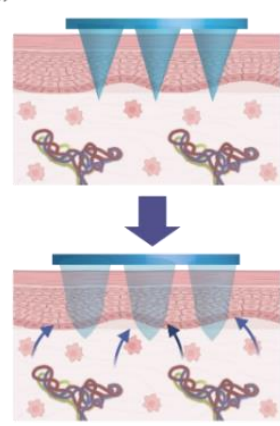

h)

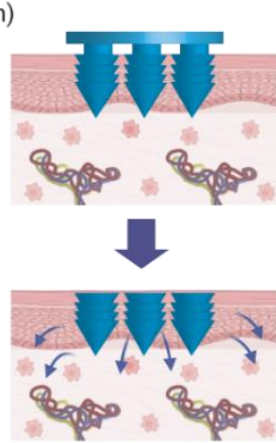

406

Figure 3. Drug release mechanisms from eight different MN types that used to facilitate intradermal drug delivery (a) Solid MNs are used to puncture the skin and are then removed, followed by application of a drug/ nanoparticles formulation. (b) Dissolvable MNs for rapid or controlled release of the drug incorporated within the MNs. (c) Nano/microparticles loaded dissolvable MNs; for intradermal implantation of drug-loaded nano/microparticles (d) Bilayer separable MN designs; drug-containing biodegradable needles are separated from the backing layer and deposited/implanted in the skin for slow drug release. (e) Drug/ nanoparticles coated solid MNs are used to puncture and skin and are placed therein. (f) Hollow MNs used to puncture the skin and enable the release of a liquid drug following active infusion or diffusion of the formulation through the needle bores. (g) Hydrogelforming MNs take up interstitial fluids from the tissue, inducing diffusion of the drug located in a patch through the swollen microprojections. h) Bioinspired MN; MNs with backwards-facing barbs that are mechanically interlocked with the skin/ocular tissue following their insertion.

\section{MNs-assisted delivery of long-acting formulations}

MNs have received a great deal of attention as a minimally invasive delivery system to deliver long-acting formulations into the biological tissues including the skin, the ocular and vaginal tissues. While pre-clinical and clinical development of MNs formulations mainly focuses on bolus drug delivery, recently MNs explored for sustained delivery to achieve long-acting treatment [99]. Particularly, polymeric MN shifted focus to controlled intradermal drug delivery because of tuneable properties of biodegradable polymers. MNs prepared from such biocompatible polymers are mechanically strong enough to penetrate the skin layers to deliver the contents intradermally where it can enable therapeutic dosing by manipulating the release 
kinetics of pre-loaded drugs. Sustained release from delivered MNs depot can be achieved by modifying the degradation and swelling profiles of the polymer composition and the diffusion profiles of the encapsulated drugs [100].

MNs-assisted delivery of long-acting drug formulation has several potential clinical benefits as documented in many references $[1,15,32,35,101]$, these include:

432 (1) Unlike, conventional long-acting parenteral therapeutic delivery often necessitates surgical implantation or hypodermic needle injection, $\mathrm{MN}$-assisted delivery of sustained-release formulations offer the potential of self-administration and as such allows the patients to apply the MNs at home without the need for trained clinical personnel.

436 (2) It does not require sophisticated set-up or electrical supply which further lowers cost and complexity compared to other minimally invasive transdermal delivery methods.

(3) MNs-assisted sustained drug delivery can minimise drug administration frequency and therefore, decrease cost. Minimising the administration frequency, thus it enhances patient compliance through improved convenience and reducing forgotten doses and lessens the risk of infection at the site of administration, especially when compared to intravenous (IV) or intramuscular (IM) injections.

(4) It helps eliminate the well-established limitations associated with hypodermic needles such as improper needle disposal and inappropriate re-use, which are of particular concern in the developing world.

(5) MNs-based sustained release systems allow using less amount of total drug to treat a diseased condition. Additionally, by reducing the total amount of drug, it can decrease in systemic or local side effects as well as the overall cost of treatment.

449 (6) Sustained-release MN makes it an excellent option for localised skin or ocular disease conditions because the drug is administered locally, right to the target site, only a small amount of the drug is needed. 
In general, the main purpose of MNs is to penetrate the skin or, any other biological tissue without breaking or bending [102]. The optimal MNs design should provide a low insertion force and high fracture force. For hydrophilic drugs, MNs that can only pierce the outermost layer of the skin would be sufficient to enhance transdermal drug delivery and drug-loaded in the MNs baseplate can be delivered by diffusing through the microchannels created by the MNs [103]. However, this is not the case for hydrophobic and long-acting formulations. For such drugs, the MNs should be able to inoculate the drug inside the skin. Consequently, any drug particles other than those loaded in the inserted part of the MNs projections will be wasted. Therefore, an appropriate and suitable MN system requires careful consideration careful of the MN geometry that further allows, sufficient mechanical properties, maximum insertion capability and, high drug loading per unit area to maximise the amount of drug which could be delivered per unit area. Moreover, MNs should be layered so that the drug should be loaded in the MNs projection, specifically in the inserted part of the projection, to minimise drug wastage and maximise the MNs delivery efficiency [104,105]. Since the dissolving MNs are the most used in delivering the long-acting formulations, therefore, this will be the focus in this section.

MNs mechanical properties, insertion capability and, drug loading capacity are governed by their geometry, including MN tip` radius, microneedle height $(\mathrm{H})$, base width $(\mathrm{W})$, aspect ratio (AR), interspacing (microneedle side- to side at the base) (IS) and shape [106]. Additionally, the materials used in the fabrication of the MNs and the skin properties further play a remarkable effect [106]. The inherent elasticity of the skin is, obviously, still a major challenge to the reproducibility of MN penetration. Some studies showed that, depending on the MNs' length, the skin could fold around them, leading to the partial or incomplete piercing of the SC [107]. In the literature, the most common shape used for microneedles design is either conical or pyramidal, with heights between $50 \mu \mathrm{m}$ and $2000 \mu \mathrm{m}$, base width ranging from $50 \mu \mathrm{m}$ to $500 \mu \mathrm{m}$ and an interspacing of $50-600 \mu \mathrm{m}$ [106]. A careful selection of these parameters is essential to produce functional MNs with the highest possible drug loading, maximum insertion capacity and acceptable mechanical strength, potentiating drug delivery efficiency. 
In terms of the MNs mechanical strength, Lee et al. found that polymeric MNs with pyramidal shapes exhibited better mechanical strength than those with conical shapes, likely due to their larger cross-sectional area at the same base width/diameter [35]. With the same MN shapes, the mechanical strength of MNs could be further improved by increasing the base width/diameter (i.e. decreasing the aspect ratio). Park et al. found that the failure force increased with shortening of the tip length and widening of the base width/diameter [108]. Later, Chen et al. showed that pyramidal chitosan MNs with the smallest aspect ratio exhibited the highest mechanical strength and the deepest insertion depth [109]. Gittard et al. also indicated that lower failure forces were required with increasing aspect ratios. Besides, Chen's group showed that there is no significant difference in mechanical strength for MN with the same aspect ratio but different dimensions [110]. This suggested that both shape and aspect ratio of MN may be the most crucial parameters in influencing their mechanical properties.

Regarding drug loading per unit area, this can be increased by either increasing the MNs density per MNs unit area, by reducing the interspacing [111,112] or increasing the length and width of the needle [105]. However, it should be noted that the undue widening of needle bases to decrease the aspect ratio or microneedles density per MNs unit area would increase the difficulties in achieving efficient skin insertion [57,105]. In one study, Larrañeta et al. showed that MN arrays with 361 (19x19) pyramidal MNs (600 x300 x $50 \mu \mathrm{m})$ (LxWxIS), have muchreduced insertion capability in a skin-simulant membrane- Parafilm $\mathrm{M}^{\circledR}$ and a neonatal porcine skin (around 50\% of the height of the MNs) in comparison with another MN array with 121 (11x11) conical MNs $(600 \times 300 \times 300 \mu \mathrm{m})(\mathrm{LxWx}$ IS), (insertion $>65 \%$ of the height of the microneedles) [112]. Also, an undue increasing needle length to increase the aspect ratio $>2$ would reduce the mechanical strength of the MNs [106].

Concerning MNs insertion capability and drug delivery efficiency, this can be enhanced by decreasing the needle tips radius, increasing the needle length or the interspacing, [106]. In the case of the needle tip radius, it is reported that the skin can be punctured more easily when the MN tip sharpness is increased. Chen et al. showed that chitosan MNs with a tip radius of $5 \mu \mathrm{m}$ were capable of achieving an insertion depth twice those with a tip radius of $10 \mu \mathrm{m}$. The authors reported that polymeric MNs with a pyramidal shape, smaller aspect ratio and, sharper tips exhibit good skin insertion [109]. Overall, it has concluded that the most desirable MN width and interspacing to attain good microneedles insertion and reasonable drug loading per unit area is $300 \mu \mathrm{m}$ and the ideal aspect ratio for good mechanical strength is $2[106,110,113]$. 
To improve MNs insertion capability, drug loading and drug delivery efficiency, and maintaining good mechanical strength, the Donnelly Research Group developed new MNs designs. In one study, Tekko et al. developed MNs design consisting of 256 (16x16) pedestal MNs/ $0.5 \mathrm{~cm}^{2}$ array with interspacing of $100 \mu \mathrm{m}$. Each MN measured $850 \mu \mathrm{m}$ in height and composed of a cuboidal base (Lx W: 250 x $300 \mu \mathrm{m}$ ) and pyramidal tips (Lx W: 600 x $300 \mu \mathrm{m}$ ) (Figure 4B) [104]. This design was used to prepare bilayer MNs with high drug loading (3 $\mathrm{mg}$ /array) of a hydrophobic commercially available cabotegravir nanosuspension loaded in the pyramidal tips only. Upon applying $32 \mathrm{~N} /$ array comparison force, the MNs exhibited excellent insertion capability in Parafilm $\mathrm{M}^{\circledR}$ and excised neonatal porcine skin (approximately $90 \%$ and $80 \%$ of the pyramidal tips inserted, respectively) and the amount of drug delivered was around $12 \%$. Consistent results were also reported upon using this MNs design to deliver other hydrophobic drugs [105,114]. To increase the amount of delivered drug further, Tekko et al. developed another new MN design with 256 (16x16) full pyramidal MNs (800 x300 x $100 \mu \mathrm{m})$ (LxWx IS) (Figure 4A) [15]. Using PVA/PVP as a polymeric matrix, bilayer MNs with high drug loading of methotrexate nanocrystals (around $2.5 \mathrm{mg} /$ array) were fabricated. These MNs exhibited sufficient mechanical strength and good insertion capability (>70\% of the height of the microneedles) and around $600 \mu \mathrm{g}$ of the loaded drug (25\% of the dose) was delivered in vivo. Again, similar results were also reported upon employing this MNs design to deliver another hydrophilic drug- crude micronized cabotegravir sodium [105]. These two MNs designs, in addition to the other three, have been evaluated to deliver a hydrophobic drug model- micronized cabotegravir sodium [105]. Within this study, the authors, created another pedestal MNs designs bigger interspacing $(300 \mu \mathrm{m})$ and a fewer number of needles per unit area (11x11 microneedles) (Figure 4C). Another three conical designs; full conical MN(Figure 4D), MNs with cylinder base and conical tips (Figure 4E), and MNs with longer full conical MNs $(1300 \mu \mathrm{m})$ and wider diameter $(500 \mu \mathrm{m})$ (Figure $4 \mathrm{~F})$, were created to identify the MN properties. In terms of the insertion capability, the authors concluded that the MNs with full conical MNs exhibited slightly better insertion capability than the full pyramidal MNs of the same geometry. Increasing the length and the width of the conical MNs led to efficient insertion yet requiring the application of higher forces. Adding a cuboidal or cylindrical base to the needles did not significantly change the insertion efficiency of those MNs. Increasing the interspacing in the pedestal MNs was slightly beneficial in enhancing the insertion efficiency of the MNs. This is most likely due to the smaller aspect ratio $(\leq 2)$ of the tips in comparison with those MNs with full conical and full pyramidal microneedles (>2). In terms of drug loading the amount of delivered drug per unit area in an excised neonatal porcine skin, the 
study revealed that the pedestal MNs (256 microneedles) has significantly higher drug loading

547 (around $3 \mathrm{mg} / \mathrm{array}$ ) than other MNs designs. However, the amount of delivered drug was 548 around (400 $\mu \mathrm{g} / \mathrm{array})$. Again, the MNs with 256 full pyramidal MNs and 256 full conical 549 MNs delivered significantly higher drug amounts (around $600 \mu \mathrm{g} /$ array) in comparison with 550 other MNs designs. Although pedestal MNs and the full conical and full pyramidal MNs of the 551 same geometry showed comparable insertion capabilities, the pedestal MNs was less efficient 552 in delivering the drug in the skin. This is probably due that the pedestal MNs are affected by 553 the skin elastic behaviour, which promotes the expulsion of the needle tips and therefore lower 554 amounts of the drug been delivered in the skin.

555

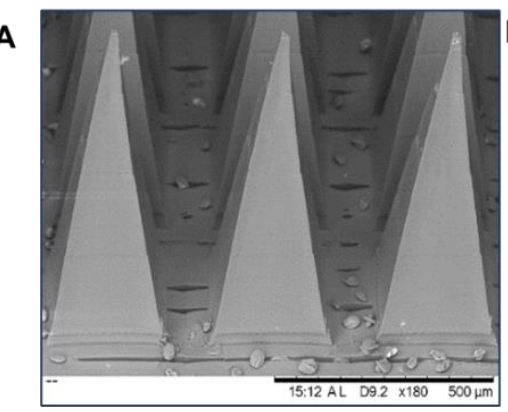

D

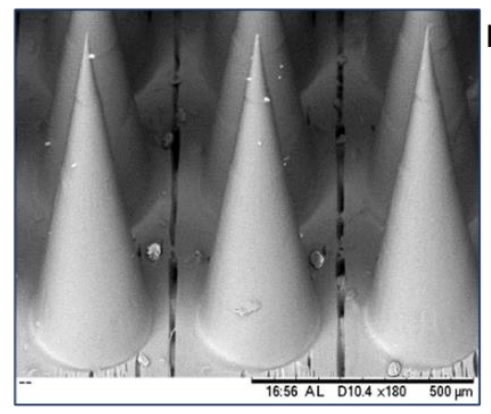

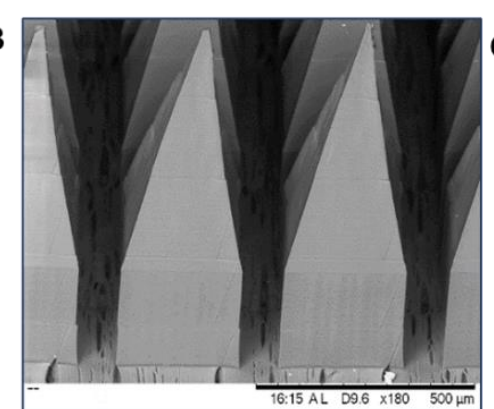

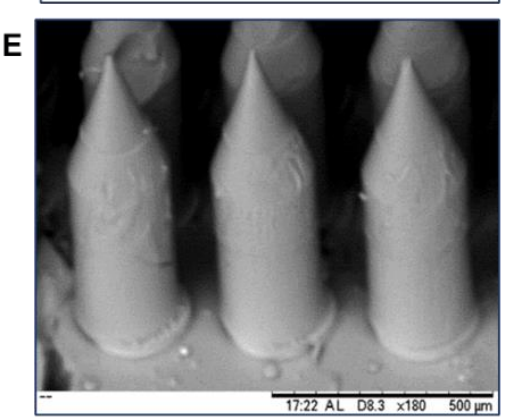

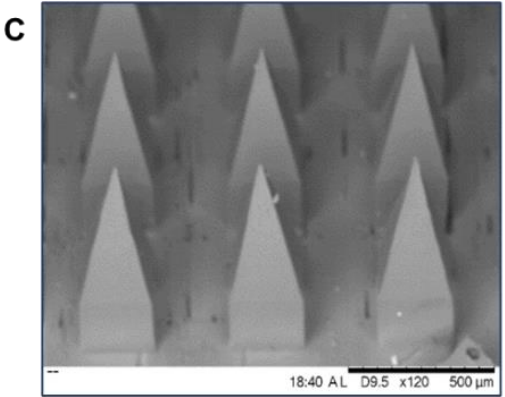

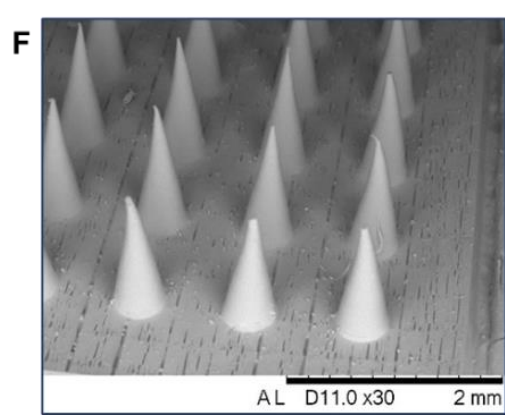

Figure 4. SEM images of six polymeric MN array designed by 3D printed $M N$ templates $\left(0.5 \mathrm{~cm}^{2}\right)$ with different geometries. A) Full pyramidal $(850 \mu \mathrm{m}) 16 \times 16$ projections. B) Cuboidal base $(250 \mu \mathrm{m})$ and pyramidal tips $(600$ $\mu \mathrm{m}$ in height) $16 \times 16$ projections. C) Cuboidal base $(250 \mu \mathrm{m})$ and pyramidal tips $(600 \mu \mathrm{m}) 11 \times 11$ projections. D) conical $(850 \mu \mathrm{m}) 16 \times 16$ projections. E) Cylindrical base $(600 \mu \mathrm{m})$ and conical tip $(350 \mu \mathrm{m}) 14 \mathrm{x} 14$ projections. F) Full conical 5x5 projections [74].

561 Based on the above-mentioned studies, it can be concluded that the optimum MNs, which could be used to deliver long-acting formulations, should fulfil certain criteria including:

(1) It should be layered, where the drug-loaded only in the MNs projections, specifically in the inserted part of the projections, and no drug in the baseplate, as this would further minimise drug wastage. 
566 (2) It should be prepared from robust materials to impart sufficient mechanical properties for 567 insertion.

568 (3) It should be prepared from biocompatible/biodegradable polymers with MW <60000 Da, 569 which allows fast clearance from the body after deposition.

570 (4) It should be long enough for transdermal drug delivery applications without causing pain $571(<1 \mathrm{~mm})$.

572 (5) It should be designed to be applied on a specific anatomical location within the body.

573 (6) It should have a certain degree of flexibility to overcome skin contours

574 (7) It should present low insertion forces and high break force.

575 (8) Importantly, the MNs geometry should overcome the skin deformation and allow maximum 576 insertion capability and high drug loading per unit area.

577 MNs with long projections (800-1000 $\mu \mathrm{m})$, appropriate projection length/width aspect ratio 578 (between 2-3) and interspacing of around $100 \mu \mathrm{m}$ could be one of the options, but focused 579 attention to the mechanical properties should be paid, to avoid such issue, MNs with pedestal 580 MNs with the same geometry could be used [104,105]. Another critical factor that should be 581 considered in $\mathrm{MN}$ design is the application method. There are two main methods of application, 582 manual and applicator based. The range of forces that both methods can apply should be taken 583 into consideration.

584 Table 1: MN-based long-acting therapeutics in pre-clinical development

\begin{tabular}{|c|c|c|c|c|}
\hline $\begin{array}{l}\text { Drug/protein } \\
\text { Molecules }\end{array}$ & $\begin{array}{l}\text { Type of } \mathrm{MN} \text { and } \\
\text { materials }\end{array}$ & $\begin{array}{l}\text { Duration of } \\
\text { release }\end{array}$ & $\begin{array}{l}\text { Application/ } \\
\text { Indication }\end{array}$ & Ref \\
\hline \multicolumn{5}{|c|}{ PLGA/PLA based separable MN } \\
\hline Levonorgestrel & $\begin{array}{l}\text { PLA arrays with } \\
\text { bubble and PVA- } \\
\text { sucrose backing layer }\end{array}$ & $\begin{array}{l}1 \text { month } \\
\text { (In vitro and in } \\
\text { vivo) }\end{array}$ & Contraceptive & {$[45]$} \\
\hline Levonorgestrel & $\begin{array}{l}\text { PLGA arrays with } \\
\text { effervescent layer and } \\
\text { PVA-sucrose backing } \\
\text { layer }\end{array}$ & $\begin{array}{l}1 \text { month } \\
\text { (In vitro and in } \\
\text { vivo) }\end{array}$ & Contraceptive & [47] \\
\hline Levonorgestrel & $\begin{array}{l}\text { Trehalose based } \\
\text { porous PLGA arrays } \\
\text { with PVA-PVP MN } \\
\text { as a backing layer }\end{array}$ & $\begin{array}{l}2 \text { weeks } \\
\text { (In vitro and in } \\
\text { vivo) }\end{array}$ & Contraceptive & [115] \\
\hline
\end{tabular}




\begin{tabular}{|c|c|c|c|c|}
\hline Etonogestrel & $\begin{array}{l}\text { PLGA arrays with } \\
\text { PVA-PVP as a } \\
\text { backing layer }\end{array}$ & $\begin{array}{l}2 \text { weeks } \\
\text { (In vitro and in } \\
\text { vivo) }\end{array}$ & Contraceptive & [116] \\
\hline Vitamin D3 & $\begin{array}{l}\text { PLGA-PLGA } \\
\text { nano/microparticles } \\
\text { loaded } P V A-P V P M N\end{array}$ & $\begin{array}{l}\text { sustained over } 5 \\
\text { days } \\
\text { (In vitro) }\end{array}$ & Micronutrient & [39] \\
\hline Doxycycline & $\begin{array}{l}\text { PLGA/PCL } \\
\text { nanoparticles loaded } \\
\text { dissolving PVA-PVP } \\
\text { MN }\end{array}$ & $\begin{array}{l}48 \mathrm{hrs} \\
\text { (In vitro) }\end{array}$ & Skin infection & [117] \\
\hline \multicolumn{5}{|c|}{ Drug micro/nanocrystals loaded MN } \\
\hline Rilpivirine & $\begin{array}{lr}\text { Pure } & \text { drug } \\
\text { nanoparticles } & \text { loaded } \\
\text { PVA dissolving MN }\end{array}$ & $\begin{array}{l}1 \text { month } \\
\text { (In vivo) }\end{array}$ & Anti-HIV & [118] \\
\hline Curcumin & $\begin{array}{lr}\text { Pure } & \text { drug } \\
\text { nanoparticles } & \text { loaded } \\
\text { PVA-PVP dissolving } \\
\text { MN }\end{array}$ & $\begin{array}{l}15 \text { days } \\
\text { (In vitro) }\end{array}$ & $\begin{array}{l}\text { Natural } \\
\text { compound with } \\
\text { a wide range of } \\
\text { application }\end{array}$ & [119] \\
\hline Methotrexate & $\begin{array}{l}\text { Pure } \text { drug } \\
\text { nanoparticles } \\
\text { PVA-PVP dissolving } \\
\text { MN }\end{array}$ & $\begin{array}{l}72 \mathrm{~h} \\
\text { (In vivo) }\end{array}$ & $\begin{array}{l}\text { Treatment of } \\
\text { psoriasis }\end{array}$ & [15] \\
\hline $\begin{array}{l}\text { Doxycycline, } \\
\text { albendazole, and } \\
\text { ivermectin }\end{array}$ & $\begin{array}{l}\text { Pure } \\
\text { nanoparticles loaded } \\
\text { PVA-PVP dissolving } \\
\text { MN }\end{array}$ & $\begin{array}{l}24 \mathrm{~h} \\
\text { (In vitro and in } \\
\text { vivo) }\end{array}$ & $\begin{array}{l}\text { Antifilariasis } \\
\text { drugs }\end{array}$ & [5] \\
\hline Cholecalciferol & $\begin{array}{lr}\text { Pure } & \text { drug } \\
\text { nanoparticles loaded } & \text { lo } \\
\text { PVA-PVP dissolving } \\
\text { MN }\end{array}$ & $\begin{array}{l}24 \mathrm{~h} \\
\text { (In vitro) }\end{array}$ & Micronutrient & [40] \\
\hline \multicolumn{5}{|c|}{ Polysaccharide/protein polymer based slow dissolving MN } \\
\hline Levonorgestrel & $\begin{array}{l}\text { Silk } \\
\text { microparticles loaded } \\
\text { or pure silk fibroin } \\
\text { MNs }\end{array}$ & $\begin{array}{l}\text { Up to } 100 \text { days } \\
\text { (In vitro) }\end{array}$ & Contraceptive & [120] \\
\hline OVA & $\begin{array}{l}\text { Sodium } \\
\text { Hyaluronate/Chitosan } \\
\text { Composite arrays } \\
\text { with PVA/PVP } \\
\text { backing layer }\end{array}$ & $\begin{array}{l}4 \text { weeks } \\
\text { (In vitro and in } \\
\text { vivo) }\end{array}$ & $\begin{array}{l}\text { Model antigen } \\
\text { for vaccine }\end{array}$ & [121] \\
\hline OVA & $\begin{array}{l}\text { Chitosan MNs with } \\
\text { PVA/PVP supporting } \\
\text { array patch }\end{array}$ & $\begin{array}{l}28 \text { days } \\
\text { (In vitro and in } \\
\text { vivo) }\end{array}$ & $\begin{array}{l}\text { Model antigen } \\
\text { for vaccine }\end{array}$ & [122] \\
\hline OVA & $\begin{array}{l}\text { Chitosan MN tips } \\
\text { with PVP coated PLA } \\
\text { supporting arrays }\end{array}$ & $\begin{array}{l}\text { up to } 14 \text { days } \\
\text { (In vitro) }\end{array}$ & $\begin{array}{l}\text { Model antigen } \\
\text { for vaccine }\end{array}$ & {$[100]$} \\
\hline BSA & Chitosan MN & $\begin{array}{l}\text { up to } 8 \text { days } \\
\text { (In vitro) }\end{array}$ & Model protein & [123] \\
\hline $\begin{array}{l}\text { Isothiocyanate- } \\
\text { dextran }\end{array}$ & $\begin{array}{l}\text { Crosslinked } \\
\text { Hyaluronic } \\
\text { nanoparticles } \\
\text { MN }\end{array}$ & $\begin{array}{l}\text { up to } 8 \text { days } \\
\text { (in vivo) }\end{array}$ & $\begin{array}{l}\text { Model } \\
\text { compound }\end{array}$ & [124] \\
\hline Insulin & Pure silk fibroin MN & Up to $60 \mathrm{~h}$ & Diabetes & [125] \\
\hline
\end{tabular}




\begin{tabular}{|c|c|c|c|c|}
\hline & & (In vitro) & & \\
\hline \multicolumn{5}{|c|}{ Lipidic nanocarriers loaded/coated dissolving MN } \\
\hline Cisplatin & $\begin{array}{lr}\text { NaCMC dissolving } \\
\text { MNs coated } & \text { with } \\
\text { lipidic } & \text { drug } \\
\text { nanoparticles } & \\
\end{array}$ & \begin{tabular}{|l}
$72 \mathrm{~h}$ \\
(In vitro)
\end{tabular} & Anticancer & [126] \\
\hline $\begin{array}{l}\text { doxycycline, } \\
\text { diethylcarbamazine } \\
\text { and albendazole }\end{array}$ & $\begin{array}{lr}\text { Solid } & \text { lipid } \\
\text { nanoparticles } & \text { loaded } \\
\text { PVP MN } & \\
\end{array}$ & $\begin{array}{l}48 \mathrm{~h} \\
\text { (In vitro and in } \\
\text { vivo) } \\
\end{array}$ & $\begin{array}{l}\text { Antifilariasis } \\
\text { drugs }\end{array}$ & [127] \\
\hline \multicolumn{5}{|c|}{ Hydrogel forming MN with drug reservoir } \\
\hline $\begin{array}{l}\text { Atorvastatin calcium } \\
\text { trihydrate }\end{array}$ & $\begin{array}{lr}\text { Liquid drug reservoir } \\
\text { with } \\
\text { (methylvinylether-co- } \\
\text { maleic } & \text { acid) } \\
\text { crosslinked } & \text { hydrogel } \\
\text { MN } & \end{array}$ & \begin{tabular}{|l}
$24 \mathrm{~h}$ \\
(In vitro)
\end{tabular} & $\begin{array}{l}\text { Cardiovascular } \\
\text { diseases }\end{array}$ & [128] \\
\hline $\begin{array}{l}\text { metformin } \\
\text { hydrochloride }\end{array}$ & $\begin{array}{l}\text { Lyophilised drug } \\
\text { reservoir with poly } \\
\text { (methylvinylether-co- } \\
\text { maleic } \quad \text { acid) } \\
\text { crosslinked hydrogel } \\
\text { MN }\end{array}$ & $\begin{array}{l}24 \mathrm{~h} \\
\text { (In vitro and in- } \\
\text { vivo) }\end{array}$ & Diabetes & {$[64]$} \\
\hline Methotrexate & $\begin{array}{l}\text { Patch drug reservoir } \\
\text { with PVA/PVP-based } \\
\text { hydrogel-forming } \\
\text { MNs }\end{array}$ & $\begin{array}{l}24 \mathrm{~h} \\
\text { (In vitro and in- } \\
\text { vivo) }\end{array}$ & $\begin{array}{l}\text { Rheumatoid } \\
\text { arthritis (RA) }\end{array}$ & [129] \\
\hline
\end{tabular}

\section{2. $\quad$ MNs formulation approaches for long-acting drug delivery}

587 Lee et al. for the first time proposed the potential use of dissolving MN for sustained delivery from the backing layer of the MN. They achieved sustained delivery ranging over hours to days by encapsulating a model dye within the MN backing, which served as a controlled release reservoir. This reservoir delivered molecules by a combination of swelling the backing with interstitial fluid extracted out from the skin by inserted $\mathrm{MN}$ and molecule diffusion into the skin via channels formed by dissolved MN. Moreover, they demonstrated that modifying MN patch matrix composition can modulate the release kinetics in human cadaver skin ex-vivo.

594 Thus, MNs offer the important potential to be able to change release kinetics based on patch type and materials, to necessitate different release profiles in order to achieve the optimum therapeutic effect for different drugs administered for various indications [35]. Hormones and other biomolecules (peptides and proteins) often necessitate a sustained release for a prolonged time, which is achievable using polymeric MN technology. PLA, PLGA or poly(caprolactone) (PCL), are often used for sustained drug release because of their different intrinsic properties $[39,130]$. Slow dissolving MNs are also produced using biodegradable naturally derived 
601

602

603

604

605

606

607

608

609

610

611

612

613

614

615

616

617

618

619

620

621

622

623

624

625

626

627

628

629

630

631

632

biopolymers, including silk fibroin and chitosan, to form the $\mathrm{MN}$ matrix to achieve the sustained therapeutic effect $[81,131]$. After skin insertion, they slowly degrade/dissolve, then releasing their drug cargo that may be sustained for days to weeks depending on the molecular weight and method of fabrication [35,36]. Dissolving polymeric MN can maintain slower drug release over a prolonged period by loading the particulate (nano/microcrystals) form of drugs, thanks to intrinsic slow dissolving properties of the drug particles [119]. Some approaches where drug-loaded lipidic or polymeric nanocarriers encapsulated in dissolving polymeric MN for intradermal deposition for slower or targeted drug release [39]. MNs can take advantage of chemical or biological triggers, to efficiently turn drug dosing on and off, to manipulate the properties of the polymer network or encapsulated drugs to attain sustained release $[132,133]$. Some of the MN-based long-acting therapeutics by use of different slow dissolving polymers and nanoparticles that in pre-clinical development is described in Table 1. In the following section, we draw attention to different approaches to achieve controlled/sustained drug release from MNs, either by controlling the release kinetics or targeting specific sites.

\subsubsection{Nano/microparticles combined with MN}

\subsubsection{Polymeric Nano/microparticles loaded MN}

Polymer-based, controlled release particulate systems include, solid colloidal nanoparticles (10-500 nm) or microparticles ( $\geq 1 \mu \mathrm{m})$ and, typically comprise of bioactive materials that are internalized through dissolving, wrapping, or adsorption and adhesion on the surface of the particles [89]. Nano/microparticles offer several benefits over conventional drug delivery systems. They permit sustained drug release over a prolonged period of time while protecting encapsulated materials against chemical or proteolytic degradation [134,135]. A best possible approach to increase the permeability of such sustained-release particulate system through the skin/eye is through the combined use of MNs. The development of combinatorial approaches to maximise the advantages of both particulate and MN delivery systems has increased over the past two decades [11].

Vora et al. reported this Vora et al. reported this combinatory approach of PLGA nano/microparticles loaded PVP MN with a model drug (Vitamin $\left.\mathrm{D}_{3}, \mathrm{VD}_{3}\right)$. The MN bilayer structure was prepared from PLGA nano/microparticles with PVP hydrogel by centrifugation technique, to concentrate the particles in MN arrays (Figure 4a) that allow to deliver most of the drug loaded particles intradermally (Figure 4b). This PLGA nano/microparticle was prepared by single emulsion solvent evaporation technique. The multisize particles displayed 
triphasic release kinetics that included initial burst release of drug from nanoparticles and drug on the surfaces of particles as well as sustained-release (more than 7 days) dependent on microparticle polymer degradation (Figure 4e). SEM images (Figure 4d) showed nano/micorparticles specifically concentrated in MN tips and compactly packed. There was a discrete line between PLGA particles mixed with PVP polymer and plain PVP polymeric matrix clearly visible. After MN insertion in full-thickness porcine skin ex vivo, as shown in optical coherence tomography images (Figure 4c), particles embedded MN exhibited a 4.9-fold higher intradermal drug deposition compared to a needle-free patch [39]. This novel bilayer $\mathrm{MN}$ concept with slow releasing drug particles or polymeric particles concentrated tips could assist to deliver the maximum load and avoid the wastage of drug formulation.

\section{a}
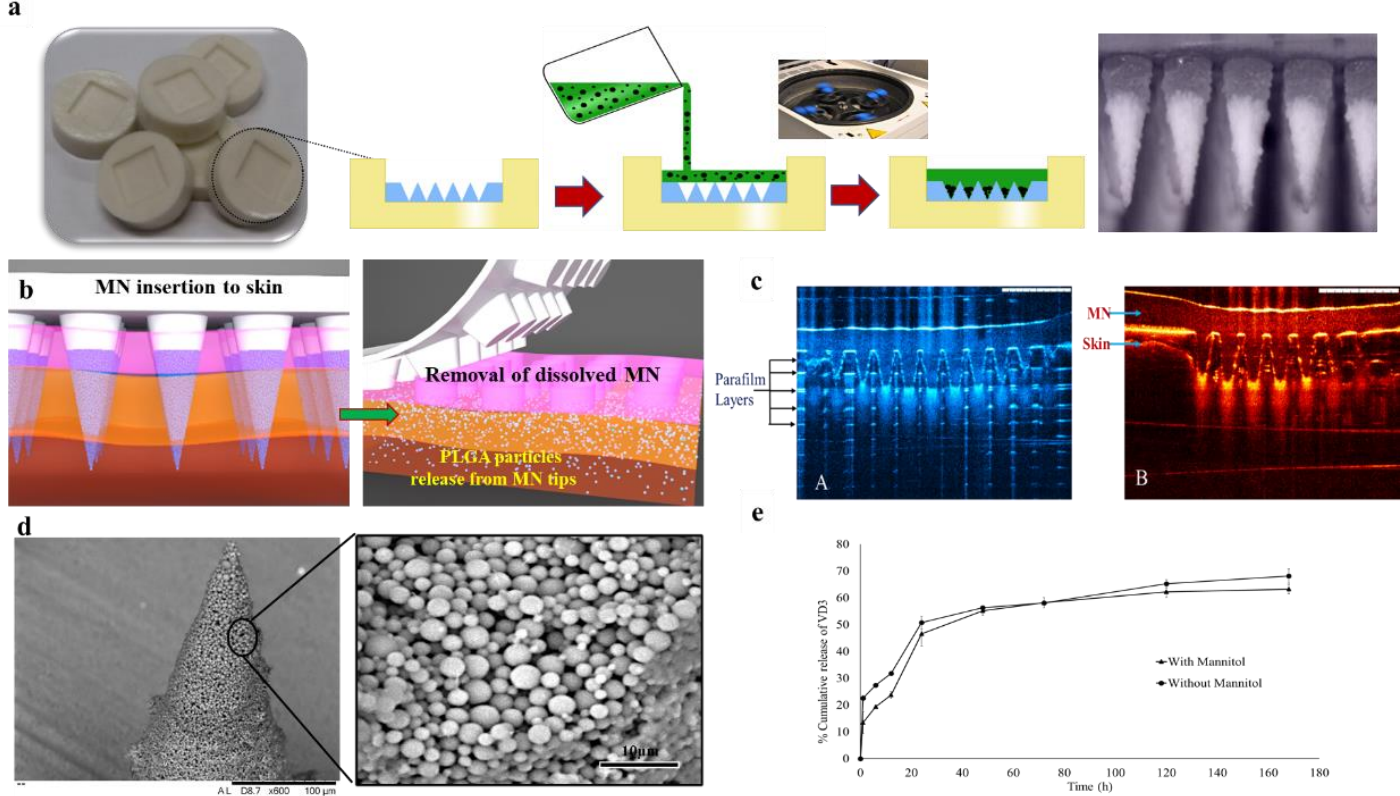

e

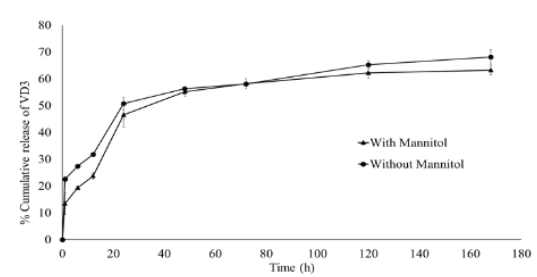

Figure 4. PLGA nano/microparticle-loaded bilayer MN arrays for sustained delivery. a) Schematic representation of MN fabrication. b) Schematic representation of MN skin application. c) An optical coherence tomography image of MN inserted in Parafilm $\mathrm{M}^{\circledR}$ and neonatal porcine skin. d) Scanning electron micrograph (SEM) of PLGA nano/microparticles-loaded bilayer MNs and e) In-vitro drug release from PLGA nano/microparticles. Reproduced with permission [39]. Copyright 2017, Elsevier.

\subsubsection{Lipidic nanoparticle loaded $M N$}

The studies of lipid-based drug nanocarriers started in the early 1960s with the development of the liposomes $[136,137]$. It was several decades later when alternative lipid nanocarriers like 
these methods are designed to encapsulate the drug, protecting it from degradation, controlling its release in a rate and site-specific manner [138-141]. Therefore, their use in conjunction with other drug delivery systems such as MNs results in an attractive new target for scientific exploration, as reviewed in this section. The first liposome-based MN system was reported in 2008 [142]. In an in vitro study, the authors pre-treated porcine skin with solid MNs and the applied deformable docetaxel liposomes, showing that the innovative treatment was able to improve the in-skin delivery of the drug. Also using a combination of liposomes and MNs, Chen et al., augmented the plasma exposure of the anti-arthritis drug triptolide in comparison to oral treatment in rats [143]. In this work, the authors were also able to demonstrate an improved efficacy in vivo, where both joint swelling and immune markers were significantly reduced. Lee et al. evaluated the potential for sustained release of NLCs loaded into MNs [144]. To this purpose, the NLCs loaded with the model drug Nile red was placed into dissolving MNs made of hyaluronic acid. After a $24 \mathrm{~h}$ release study performed in Franz cells using dermatomed minipig skin, a radial spread pattern was observed, with $70 \%$ of the drug located in the skin. Using a similar approach, aconitine NLCs were loaded into dissolving MNs and assayed in a rat model of arthritis, where a significantly improved efficacy and decreased drug toxicity were observed [145]. The MNs seemed to form an in-skin drug reservoir, suggesting that the novel experimental formulations have the potential to release the active sustainably [28]. An interesting proof-of-concept study reported by Permana et al. aimed to target the lymphatic system with the conjunction of SLNs and to dissolve MNs [146]. The lipid-based nanocarriers ( size $<100 \mathrm{~nm}$ ) loaded with the antifilarial drugs doxycycline and albendazole sulphoxide were vehiculised in MNs formed by PVP and PVA and tested in vivo. The biodistribution assays revealed that the drugs effectively accumulated in the lymph nodes in concentration levels 4- and 7-fold higher than those observed for the control groups treated with oral formulations.

\subsubsection{Metallic nanoparticles loaded MN}

Inorganic nanoparticles, such as iron oxide nanoparticles (IONPs), gold nanoparticles (AuNPs), silver nanoparticles (AgNPs), quantum dots, silica nanoparticles and, carbon nanotubes, have been extensively investigated for their potential role in drug delivery $[147,148]$. Some of the unique physical and biological properties of these particles may allow the development of multi-function particles that could be used for diagnostic and therapeutic ("theranostic") purposes [85]. Magnetic IONPs have been used to deliver ARV drugs due to their unique features, namely, controllable size distribution, biocompatibility, ability to bind 
687

688

689

690

691

692

693

694

695

696

697

698

699

700

701

702

703

704

705

706

707

708

709

710

711

712

713

714

715

716

717

718

drug and sustain drug release, magnetically-guided site-specific targeting, and the ability to convert magnetic fields to heat energy. Saiyed et al. made 3'-azido-3'-deoxythymidine-5'triphosphate (AZTTP) bound onto magnetic $\mathrm{Fe}_{3} \mathrm{O}_{4}$ nanoparticles by ionic interaction; the magnetic nanoparticle-bound AZTTP maintained its biological activity in peripheral blood mononuclear cells, and active NRTIs were targeted to the brain by applying an external magnetic force [86]. Magnetic IONPs could also be used for magnetic resonance imaging (MRI) to monitor drug distribution to target tissues in vivo. Superparamagnetic iron oxide nanoparticles (SPIONs) have shown to have the ability to track HIV-specific cytotoxic T lymphocytes (CTLs) [88] and to increase the cytotoxic potential of HIV-specific CTLs and improve the killing of HIV-infected cells in latent HIV reservoirs by employing magnetic field hyperthermia effects [89].

\subsubsection{Nano/micro drug crystals loaded MN}

The manipulation of particle size has been primarily used in the pharmaceutical industry to increase the dissolution rate of poorly water-soluble drugs (solubility $<0.1 \mathrm{mg} / \mathrm{mL}$ ) [149]. Firstly, the production of drug microparticles $(<10 \mu \mathrm{m})$ was achievable mainly using comminution techniques. However, the development of novel formulation methods permitted the formation of drug particles within the sub-micrometre range, namely nanocrystals (NCs) [150]. NCs are drug nanoparticles with crystalline features, with minimal carrier materials or excipients, therefore capable of attaining a drug loading of close to $100 \%$ [151]. The term nanosuspension refers to the NCs dispersed in a liquid, whereas solid and re-dispersible NCsbased powders can be obtained by solvent removal [152-155]. NCs can be produced by two main approaches, "bottom-up" techniques, which consists of controlled drug precipitation from a solution, or by "top-down" techniques, which include the application of external shear forces [156]. Distinctive features of NCs include increased saturation solubility, dissolution rate and mucoadhesion, which leads to greater absorption and efficacy [157-159]. These advantages, coupled with the availability of flexible and scalable manufacture techniques, make of NCs an attractive option for the formulation of poorly soluble drugs.

The intradermal administration of micronised drugs and NCs using MNs has been reported in several recent publications. For instance, Vora et al. prepared a nanosuspension of the hydrophobic vitamin cholecalciferol by sonoprecipitation technique. The resultant nanosized suspension (average diameter of $305 \mathrm{~nm}$ ) was loaded into dissolving MNs, which presented a significantly higher ( 7 fold) drug permeation ex-vivo, across dermatomed (350 $\mu \mathrm{m})$ neonatal 
porcine skin over $24 \mathrm{~h}$ [10]. Similarly, intending to potentially treat a series of local and systemic diseases, Abdelghany et al. loaded curcumin containing NCs into dissolving MNs [160]. In this work, the authors reported enhanced permeation of curcumin from the novel MN systems in comparison to the control (topical application). In a different report, NCs loaded with the antifilarial drugs doxycycline and ricobendazole (mean diameters below $100 \mathrm{~nm}$ ) were incorporated into dissolving MNs composed of PVP and PVA [161]. In vitro studies demonstrated that the experimental $\mathrm{MN}$ formulations delivered the actives more efficiently than the control formulation consisting of a needle-free patch. In further studies, the contraceptive drug etonogestrel was loaded into dissolving MNs with the intended purpose of prolonged-release [162]. Here, drug microparticles were blended with aqueous solutions of PVA to cast the MNs. The authors demonstrated the ability of the novel formulations to deliver the drug for one week. Additional studies conducted by McCrudden et al. loaded an industrially prepared nanosuspension of the antiretroviral drug rilpivirine into dissolving MNs to develop a sustained-release formulation with improved patient compliance [163]. After successful mechanical characterisation, MNs were tested in vivo in Sprague-Dawley rat models, where they exhibited similar, if not superior pharmacokinetic behaviour to an intramuscular injection of the same nanosuspension. Based on the promising results obtained, the same researchers used a similar approach to deliver rilpivirine to the vaginal tissue, for the first time [164]. In vivo studies demonstrated that the mean plasma concentrations of the drug at the endpoint of the study (day 56) were similar to that of the cohort treated with the intravaginal MNs (116.5 $\mathrm{ng} / \mathrm{mL})$ and IM injections (118.9 $\mathrm{ng} / \mathrm{mL})$.

Itraconazole NCs were prepared by media milling using Poloxamer 407 as a stabilizer were incorporated into dissolving MAPs fabricated with PVP/PVA. The novel formulations were successfully characterised in terms of mechanical and physicochemical properties and afterwards tested in an in vitro model of skin candidiasis. This experiment demonstrated a significantly increased efficacy against the fungus when compared to control cohorts [114]. The advantages and potential applications of dissolving MNs and nanocrystal technologies have been thoroughly discussed in a recent review article [165].

The sustained release of dutasteride from dissolving MNs was reported by Giffen et al. In this work, the authors used a nanosuspension obtained by media milling and a blend of CMC, trehalose and polysorbate 80 to microfabricate the MNs [166]. Following optimisation formulation, a pharmacokinetic trial was conducted in rats and minipigs using IV and intradermal injections as respective controls, where they proved that the MN-based formulation 
significantly increased the plasma half-life of the drug. These results together, indicate that the combination of micronised or nanocrystalline drugs with MNs is an appealing strategy for the sustained release of drugs via intradermal delivery. Dissolving MNs, in particular, are relatively simple to manufacture and, they have a demonstrated ability to deliver long-acting nanosuspensions importantly, do so without the generation of sharp waste [167].

Recently, Tekko et al. combined NCs and MN technology to localise and sustain the release of methotrexate (MTX) as a potential treatment for psoriasis in order to minimise the systemic exposure in order to avoid the drug systemic toxicity and side effects. The authors prepared a poorly water-soluble MTX-NC from its freely water-soluble salt MTX Na by a bottom-up method, namely, acid-base neutralisation accompanied with sonification. The MTX-NCs (average diameter of $700 \mathrm{~nm}$ ) were then incorporated into the projections of dissolving MNs with a drug loading of $2.48 \mathrm{mg} /$ array. The MNs projections dissolved within 20 minutes postMNs application and deposited their payload in the skin. An in vivo study in Sprague Dawley rats revealed that the MTX-NCs-loaded MNs were capable of depositing approximately $25 \%$ of the loaded MTX NCs in the skin. This in turn then acted as a drug depot and released the drug in a sustained manner over $72 \mathrm{~h}$ while minimising MTX systemic exposure in comparison with orally administered MTX Na (control) [14].

\subsubsection{Slow dissolving MN}

Biodegradable polymeric MNs were developed as a method for achieving sustained transdermal drug release. These MNs have potential as a patient-friendly substitute for conventional sustained release methods [168]. Most commonly used biodegradable MNs are described below, whereby the release of incorporated drugs can be sustained for days to weeks [169].

\subsubsection{PLGA/PLA MNS}

Biodegradable polymers of the aliphatic polyester family are PLGA and PLA, which have already been frequently applied in tissue engineering and drug delivery. PLGA, a copolymer of PLA and PGA, is the best defined biodegradable polymer in the controlled release of encapsulated drug and, is widely used for sustained drug release at desirable doses by implantation without surgical methods $[170,171]$. Additionally, the overall physical properties of the polymer-drug matrix can be tuned by controlling the relevant factors such as the ratio of lactide to glycolide, drug concentration and, polymer molecular weight to achieve a desired 
dosage and release period varying upon the drug type [170]. Furthermore, both polymers are well-defined in safety, with various drug products containing PLGA approved by regulatory authorities and available for use in clinical practice [172]. Thanks to high biocompatibility, strong mechanical properties, and safe profiles, PLGA and PLA have already been employed in MNS fabrication for long-acting sustained delivery. As PLGA and PLA have a high melting point, heating at a high temperature above $135^{\circ} \mathrm{C}$ under vacuum is generally required to fill the microcavities of the moulds $[133,173]$. This relatively high temperature makes the fabrication method unfavourable, especially for the delivery of heat-sensitive compounds. MNs fabricated by drug PLGA and/or PLA mixture, are mostly manufactured through the solution casting method. Some of the most commonly employed solvents utilised in this process include: dioxane, tetrahydrofuran [45], N-methyl pyrrolidone [115], dimethylformamide [174], ethyl acetate [48] and dimethyl carbonate [48], diglyme [47] and, DMSO [175]. At times, water may also be added to the process in order to adjust the rate of the evaporation [45]. These organic solvents were removed by evaporation or, at $75-100^{\circ} \mathrm{C}$ within a vacuum oven $[45,115,175]$. Another commonly employed method to prepare PLGA and/or PLA MN is spray deposition. Researchers employed a spray disposition process for sequentially spraying of PLGA and PVP solution into the mould. However, it must be taken into consideration, that the use of organic solvent and heating might affect the structure and bioactivity of biomacromolecules during formulation $[48,176]$. Typically, therapeutics loaded in the PLGA tips are mainly hydrophobic compounds, such as levonorgestrel [45], etonogestrel [116], and rhodamine [46], predominantly the solubility of the mixture of drug and polymer in organic solvents. However, despite this, the hydrophilic $\beta 3$-adrenoceptor agonist CL316,243, has been incorporated within the system [174]. Biomacromolecules such as ovalbumin, bovine serum albumin and horseradish peroxidase were also loaded into this type of MN system, and it was found the protein retained $80 \%$ of the bioactive despite the addition of organic solvents. Interestingly, one study demonstrated the successful loading of mesenchymal stem cells into PLGA MN shells. This system consisted of an array of MNs with an outer PLGA shell and an internal gelatin methacryloyl-mesenchymal stem cells mixture. This device exhibited enhanced wound healing by localizing mesenchymal stem cells delivery with a minimal dose of cells [177].

To shorten the patch application time and quicken the implantation of PLGA/PLA needle tips, various two-layers and three-layer MN designs were attempted. Most researchers have used the dissolving material as a baseplate to separate them from insoluble PLGA/PLA tips $[45,116,174]$. As the upper part of the water soluble tips dissolves in the skin fluid, the PLGA 
tips were separated and implanted inside the skin. The freeze-drying method is also employed

817

818

819

820

821

822

823

824

825

826

827

828

829

830

831

832

833

834

835

836

837

838

839

840

841

842

843

844

845

846

847 to make the baseplate backing layer more hygroscopic to fasten the dissolution time required for quicker PLGA/PLA tips separation [46]. Prausnitz et al. recently developed rapidly separating/biodegradable MN, made of PLA and PLGA for the continuous release of levonorgestrel (LVN). In this study, the authors attempted to develop an air entrapped bubble structure between each PLGA/PLA MN and the water-soluble patch backing while fabricating the patches (Figure 5ia). Bubble structures allowed the MN to penetrate the skin upon compression, and to be 'snapped off' within 5 seconds of application. The MNs then remained embedded beneath the skin surface. The drug release is then controlled by a combination of diffusion and matrix erosion. The authors demonstrated that rats tolerated the MN patch and that upon removal it left a little visible residue, and that it maintained plasma concentrations of the hormone above the human therapeutic level (200 pg/ml) for one month (Figure 5ib) [45]. Moreover, the authors evaluated effervescent formulations that sandwiched between the PLA MN tips and the patch for in-situ effervescent generation and faster separation during skin insertion (Figure 5iia) [47]. Upon application to the skin, the PLGA and PLA MN tips implanted in the viable tissue, depositing the therapeutic cargo, from which sustained drug release is achieved (Figure 5iib). The release time has been reported to vary from days to 3 months $[45,46,178]$. The release rate of the PLGA/PLA tips could be affected by the amount of drug loading [178], the polymer chain composition [174] and, the addition of porogens, for example, trehalose [115]. It was found that the $10 \% \mathrm{w} / \mathrm{w}$ drug (2-methoxyestradiol) loaded PLGA MN implants released the drug completely within 8 weeks, whereas the $25 \% \mathrm{w} / \mathrm{w}$ drug loaded MN tip implants maintained therapeutic levels (0.1-1 mM) for 90 days. As reported by Aung Than et al., drug-release kinetics of PLGA-MNs loaded with Cy5 could be readily modulated by choosing or mixing different PLGA molecules with different structure, chain length, and copolymer ratio [174]. Moreover, the addition of trehalose as a porogen in PLGA MN tips could tailor the faster release of LVN [115].

The most challenging issue with this system is the limited dosage. As reported in the literature, the dose loaded in a $10 \times 10$ or $11 \times 11$ arrays $/ 0.5 \mathrm{~cm}^{2}$, were not able to exceed $0.5 \mathrm{mg}[45,116]$. Because of this, only very potent drugs such as hormones are appropriate for delivery by this system [39]. Vaccine antigens were also loaded in the system. However, the use of organic solvent or heat might adversely affect the protein structure. Localised therapies are also recommended for this system for future study. 


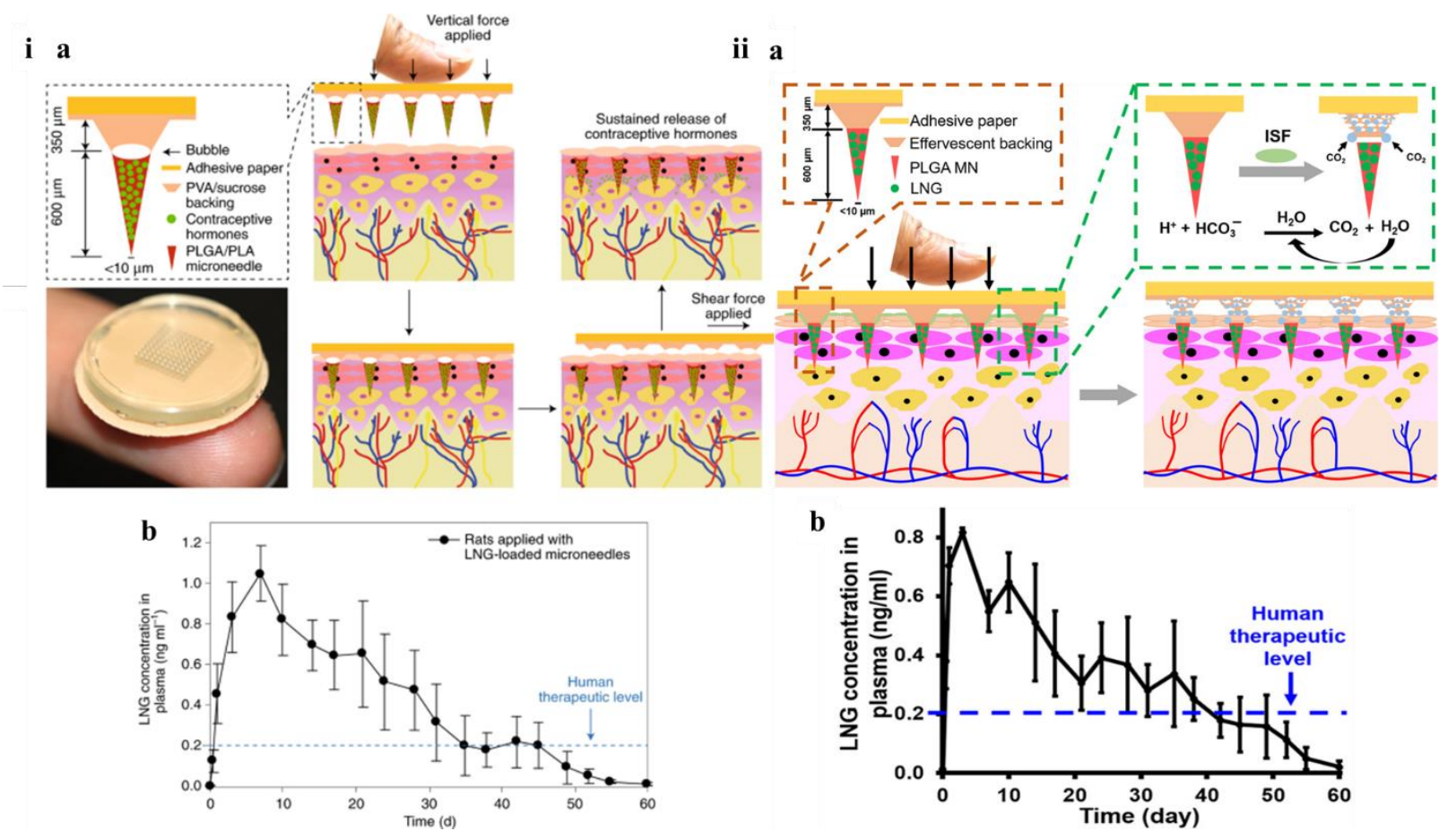

Figure 5. A rapidly separable PLGA/PLA MN patch for the prolonged delivery of the contraceptive LVN, i) a) Bubble ii) a) effervescent MN design with the skin application process for sustained release of a contraceptive hormone. ia) and iib) Concentration of LVN in the plasma of rats after administration of the LVN-loaded MN patches with respective MN designs, as a function of time. Reproduced with permission [45,47]

\subsubsection{PCL MNS}

As a biodegradable and non-toxic material, PCL has been extensively utilised in a wide range of drug-delivery devices, notably in the biomedical applications of controlled-release drug delivery systems, absorbable surgical sutures, and three-dimensional scaffolds [179]. Attempts have also been made to utilise PCL for MN fabrication owing to its good solubility, low melting point, and exceptional blend-compatibility [180]. Despite a relatively low water solubility, PCL exhibits favourable solubility in organic solvents. Therefore, the solution casting method has been widely used in PCL MN fabrication. Generally, PCL is mixed with the drug in chloroform, acetone, or dichloromethane and, methanol. After the solution is cast on the moulds, the organic solvents are evaporated. A separable baseplate is often added to the PCL tips to enable the complete implantation of PCL drug-loaded tips. PCL/PEG blends tips MN were fabricated using the solution casting method. One of the most attractive advantages of PCL is its low melting point. The relatively low melting point of PCL made it possible for hot embossing PCL and drugs into the MN. PCL MN containing 20\% furosemide was fabricated by the hot embossing method, and the release of furosemide was demonstrated by diffusion [181]. The low melting point of inserted PCL MN tips also provides opportunities for triggered 
869

870

871

872

873

874

875

876

877

878

879

880

881

882

883

884

885

886

887

888

889

890

891

892

893

894

895

release behaviour to manufacture the stimuli-responsive MN. The inserted PCL MN tips were able to transit the thermal phase from a solid to a liquid at a relatively lower temperature such as $50^{\circ} \mathrm{C}$. The thermal phase transition of PCL increases the mobility of the polymer chain and triggers drug release from PCL [182]. An electron-heating sheet was used to provide the heat to change the phase of implanted PCL MN tips. The melting of PCL tips was able to thermally modulate the release of the encapsulated metformin [183]. Besides, the heat for melting PCL tips can also be provided by near-infrared light and photosensitive materials. Photosensitive materials such as lanthanum hexaboride [184,185], silica-coated lanthanum hexaboride [182,186], Prussian blue nanoparticles [187], and $\mathrm{Cu}_{7} \mathrm{~S}_{4}$ nanoparticles [130] were entrapped inside the PCL tips with drugs such as metformin [187], lidocaine [185] or DOX hydrochloride $[182,184,186]$. With the activation of near-infrared light, photosensitive materials were able to produce enough heat to melt the PCL tips. Thus, the release behaviour of the drugs can be precisely controlled by switching on and off the near-infrared light. Furthermore, magnetic nanoparticles can produce heat under an alternating magnetic field due to hysteresis loss. Biodegradable $\mathrm{Fe}_{3} \mathrm{O}_{4} / \mathrm{PCL}$ MNs were developed for potential skin cancer treatment with transdermal controlled drug delivery and magnetic hyperthermia [188].

\subsubsection{Silk MNs}

Silk-based biomaterials have excellent mechanical properties, biocompatibility, biodegradability, and the capability to protect and maintain the activity of biomolecules $[189,190]$. The degradation rate of silk-based materials and the diffusion rate of the encapsulated molecules can be self-controlled by adjusting post-processing conditions through the silk protein secondary structure [191]. Another advantage is that biomolecules or labile drugs can be incorporated into the silk-based matrix as a result of the aqueous and mild processing conditions by nano and microfabrication [14]. It is also well proven that biomolecules can be stabilised in dry silk films for extended periods [192]. The attractive properties afforded by silk biomaterials make it an ideal choice for the MN-based sustained and long-acting delivery system [131]. 

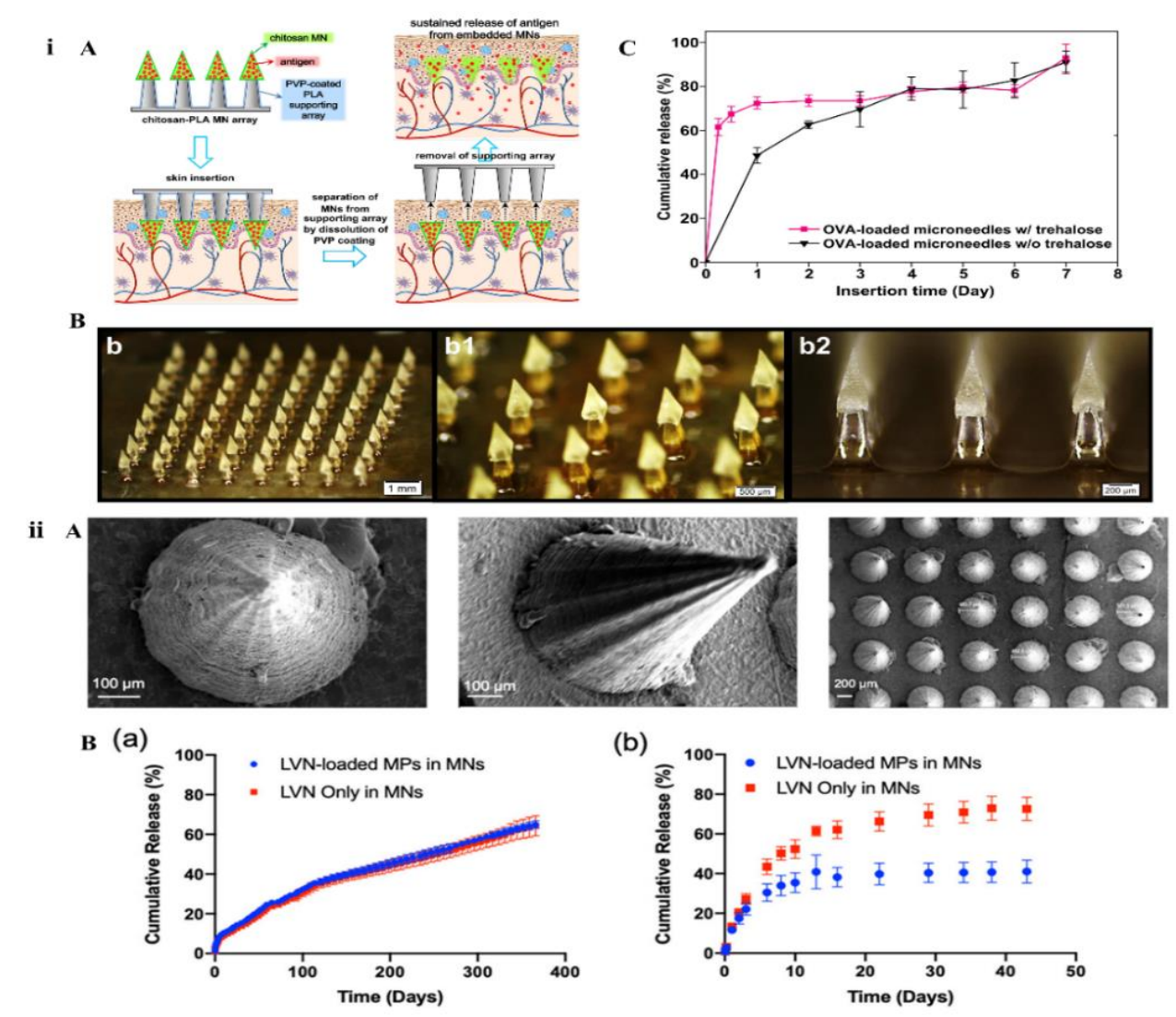

897 Figure 6 i) A) Schematic illustrations of sustained transdermal delivery of antigen using MN delivery system, composed of embeddable chitosan MNs and PLA supporting array. B) Bright-field micrographs of ovalbuminloaded (b-b2) chitosan-PLA MN arrays/ C) In vitro release profiles of OVA-loaded MN with or without trehalose, conducted using Franz diffusion cells. Reproduced with permission [81] Copyright 2017, Elsevier. ii) A) SEM micrographs showing the structure and size of LVN -loaded silk MNs. B) In vitro release profiles of LVN from silk MN patches in (a) $1 \mathrm{~mL}$ PBS, (b) $200 \mathrm{~mL}$ of $0.5 \%$ SDS solution. Reproduced with permission [120] Copyright $\odot 2020$ American Chemical Society.

904 Yin et al. developed a 2-ethoxyethanol (ECS) modified silk fibroin (SF) MN with swelling characteristic to generate 3-dimensional (3D) porous network for sustained transdermal delivery. This modified SF MN was strong enough to pierce excised porcine skin and transform into semi-solid hydrogels with 50-700 $\mathrm{nm}$ porous network inside and, thus, provide substantially better transdermal drug release [193]. The release rate from silk-based MNs can be further regulated by altering the crystallinity. Wang et al. used the nontoxic proline treated SF to obtain MNs with Silk I crystal structure (random coils to $\beta$-sheets). Within this study, different ratios of proline to change the crystallinity of fibroin were evaluated, in order to improve the needles' swelling capacity upon encounter with the intradermal interstitial fluid.

913 The needles were strong enough to penetrate the $S C$ and created the microchannels. In vitro, 914 insulin release experiments showed that the release rate of proline/fibroin MNs increases based 
on the degree of swelling. The cumulative insulin release rate reached up to $80 \%$ with more than $60 \mathrm{~h}$ long sustained release that indicates a relatively high drug utilisation rate. Thus,

917 potentially offering an effective and painless potential treatment route for diabetic patients

918 [125]. Tsioris et al. reported a fabrication method to produce silk biopolymer microstructures

919 with the necessary high aspect ratios required to fabricate MNs. Room temperature and aqueous-based micromolding allowed for the bulk loading of these MN structures with temperature-sensitive drugs such as peptides and vaccines, antibiotics, or any temperaturelabile drug molecules. Controlled release of a model drug is attained by modifying the postprocessing conditions of the MN structures, mainly by controlling the silk protein secondary structure [131]. Yavuz et al. recently fabricated silk protein MN for the long-acting transdermal delivery of contraceptive hormone, LVN. Within the study, a comprehensive approach with silk MN was taken to tailor the release from months to more than a year (Figure 6ii). The authors achieved different sustained release kinetics by different loadings of the drug, via pre-encapsulation in silk microparticles or, freely inside the silk MNs. Sustained drug release extended up to 100 days when the drug was loaded directly inside the silk MN. However, the release continued for more than a year when the drug was encapsulated inside silk microparticles prior to fabricating the MN. As depicted in Figure 5B(a), drug release was controlled by drug solubility and no significant differences were found between the drugloaded microparticles in the MNs and the drug-loaded MNs in terms of release rates under nonsink conditions. However, under sink conditions, the effect of the microparticles on release rate was observed over the same period of time (Figure 6B(b)). They also employed different molecular weights of silk as well as different silk concentrations (7 and 10\%) to provide more control over the release profile. The higher the concentration of silk resulted in a slower release rate of the drug from MNs (cumulative release of $25.3 \pm 1.5 \%$ after 100 days). However, there was no significant effect on the release profile of the drug (cumulative release of $40 \%$ to $50 \%$ after 100 days) from MN by varying the molecular weights of silk. Not only that, they studied the effect of solubility enhancers such as $\beta$-cyclodextrin (5\% w/v) and a surfactant (Tween-20, $1 \% \mathrm{v} / \mathrm{v}$ ) with the silk-drug solution (before casting the $\mathrm{MN}$ ) on the release profile of the drug. Both formulations managed similar release profiles with comparatively enhanced release rate ( $60 \%$ ) compared to the silk MN without these drug solubilisers. Interestingly, all different

945 MN formulations released LVN level above the require daily contraceptive dose (30 $\mu \mathrm{g} /$ day) 946 [120]. These results indicate that long-acting contraception $\mathrm{MN}$ array patches are feasible to fabricate. 
949 Chitosan has been commonly used as a material in the manufacture of both nanocarriers or MN 950 for sustained release because of its well-known biocompatible and biodegradable properties 951 [123]. Another advantage of the inherent cationic charge of chitosan is that it promotes 952 interaction with negatively charged functional groups on proteins or other drug molecules 953 [194]. These unique characteristics, combined with its desirable film-forming properties, make chitosan appropriate as MN material for sustained delivery, especially for biomolecules [195]. In addition to the sustained release, chitosan possesses immune-modulating /stimulating activity, which can act as immunoadjuvant for vaccine/gene delivery [196]. Xie et al. reported a model molecule, calcein and bovine serum albumin (BSA) coated with different concertation of chitosan solution on solid MNs. The study evaluated the effect of varying matrix thickness, drug content and the matrix concertation of chitosan film upon the effect of sustained release [197]. Chen et al., first introduced in 2012, a chitosan MN patch for efficient and sustained transdermal delivery of the model protein BSA with enough mechanical strength for successful skin insertion. Investigation of the in vitro drug release of BSA from chitosan MN demonstrated sustained release of BSA for 8 days. This chitosan MN did not affect the secondary structure of BSA, and when the Alexa Fluor 488-labeled BSA loaded MN was applied to the rat skin in vivo, the fluorescence of Alexa 488-BSA was observed at a depth of $300 \mu \mathrm{m}$ under confocal microscopy. These proof of concept results suggested that the developed chitosan MN could be potentially useful for transdermal delivery of macromolecules in a sustained manner [123]. In a further study conducted by Chen et al., fully embeddable chitosan composite MNs with PLA supporting as a complete and sustained release depot of encapsulated antigen for intradermal vaccination was evaluated (Figure 6i). Chitosan MN separated from the PLA supporting array during insertion to rat skin in vivo, from which sustained the release of OVA for over 14 days without the need for a transdermal patch. The MNs fully penetrated the skin at a depth of $600 \mu \mathrm{m}$ to target the model OVA antigens to antigen-presenting cells in the epidermis and dermis [81]. Additionally, Chen et al. further examined the capacity of chitosan $\mathrm{MN}$ for low-dose immunisation with sustained manner. The system was composed of model OVA antigen-loaded chitosan MNs and PVA/PVP supporting array as an additional base to attain superior MNs insertion into the skin. The chitosan MNs exhibited a sustained antigen release for up to 28 days. In rats, applied chitosan MNs with low979 dose antigen had persistently high antibody levels for 18 weeks, greater IM injection. 
981

982

983

984

985

986

987

988

989

990

991

992

993

994

995

996

997

998

999

1000

1001

1002

1003

1004

1005

1006

1007

1008

1009

1010

1011

1012

added chitosan solution [122]. Marin et al. fabricated MNs from CMC and chitosan. The fabrication was achieved by a polyelectrolyte complex mechanism based on the electrostatic interaction between anionic $\mathrm{CMC}$ and cationic chitosan. They described that the MN exhibited decreased hydration, thus slowing the protein release from the polyelectrolyte chitosan coating [198].

\subsubsection{Metal MNs}

Metallic MNs, are typically fabricated with stainless steel, gold, silver, metallic glass [199] or based on bio-composites and, are commonly used as hollow, coated, or solid MNs [200]. Hollow metallic MNs are usually used to deliver liquid formulations while coated MN with a small amount of a substance such as vaccines, proteins and potent drugs [201-203]. Another popular approach is named as micro-needling for cosmetic purposes, including the percutaneous induction collagen therapy [204]. However, to achieve long-acting release it is necessary to associate the metal MNs with a structured nanotechnology formulation $[34,45]$ or, novel approaches as a highlighted by conductive MNs [205], which represent some of the most promising innovative approaches described for metallic MN patches. In an attempt to develop a new drug delivery system for short or long term release, Guoet et al. developed a new conductive polymeric MN containing a working electrode system integrated to an inert metal layer and a drug-loaded conductive film layer, with the purpose to deliver the drug through electrical stimulation [205]. The poke and patch approach was applied by Nayak et al. resulting in the extended-release of lidocaine microparticles, formed by electrostatic interaction between a hydrogel and water/oil emulsion under covalent crosslinking by glutaraldehyde. Stainless steel $\mathrm{MN}$ arrays were used to deliver lidocaine microparticles, achieving 2-fold delivery improvement when compared to the application of the same formulation without preceding treatment of the skin using metal MNs [203]. Koutsonanos et al. developed metallic MN coated with subunit influenza vaccine solution by a dip-coated method. This MN system was capable of achieving improved immune responses, conferring improved long-term protection in an in vivo mouse model compared with IM injection. Therefore, this MN method of vaccine delivery may present as a potentially beneficial approach to increase vaccine coverage and protection. The long-acting effect was observed after 36 weeks post-immunisation, where the MNs group exhibited $100 \%$ survival against $40 \%$ of mortality in the IM group [206]. The coated method is a successful strategy to penetrate the skin and easily release the compound through the skin layers, due to the small amount of drug in the tips, favouring the compound diffusion [207]. 


\subsubsection{Coated $M N$ arrays}

1014

1015

1016

1017

1018

1019

1020

1021

1022

1023

1024

1025

1026

1027

1028

1029

1030

1031

1032

1033

1034

1035

1036

1037

1038

1039

1040

1041

1042

1043

1044

The use of coated MNs for long-acting formulations is challenging due to their reduced drug loading capacity. In these $\mathrm{MN}$ formulations, the active substance may only be contained in a thin layer, which covers the surface of the MNs. Therefore, the potential application of this approach is limited to the use of highly potent agents. Despite this, some methods have been explored to control the release rate of coated MNs using diverse polymeric coatings. For example, Xie et al. coated MNs prepared by micro-electro-mechanical systems (MEMS) with a nanometre chitosan film loaded with the model drugs calcein and BSA [197]. Permeation studies demonstrated that after insertion in rat skin, the hydrophilic polymer permitted to form a matrix system through which the drug was released in a controlled manner. A similar concept was explored by Marin et al., where they obtained CMC MNs which were subsequently coated with a chitosan layer to form a polyelectrolyte complex between both polymers, thus leading to potential applications on sustained drug release [198]. In different work, lidocaine was blended with dextran and loaded onto solid MNs by dip-coating. In vivo trials in domestic swine demonstrated that the drug rapidly dissolved and achieved therapeutically relevant concentrations within one minute after the $\mathrm{MN}$ application. Moreover, the in-skin concentration of the drug was found to be above the therapeutic threshold for approximately 90 minutes [208].

\subsubsection{Stimuli-responsive smart MN arrays}

Over the last decade, stimuli-responsive polymeric biomaterials have emerged as effective systems for therapeutic delivery. The design of the material may be used to tailor smart polymers to release therapeutic payload when particular stimuli are present, which may be either internal or external stimuli triggering. As illustrated in Figure 7, internal stimuliresponsive smart biomaterials include those that respond to specific enzymes [209], mechanical force [210] or changes in microenvironment $\mathrm{pH}$ [211]; external stimuli can consist of electro or magnetic fields [212,213], light [214,215] and, ultrasound [216]; with some smart biomaterials responding to multiple stimuli. As a result, stimuli-responsiveness may potentially enhance the spatiotemporal specificity of therapeutic release, thus resulting in increased efficacy, while simultaneously reducing side effects and off-target toxicity.

In recent years, smart biomaterials have been increasingly employed in $\mathrm{MN}$ mediated delivery of therapeutics. Incorporation of stimuli-responsive units into $\mathrm{MN}$ can be exploited to trigger both on-demand and sustained drug release by either manipulation of external stimuli, or 
exploitation of naturally present internal stimuli. Hardy et al. developed a hydrogel-based MNmediated transdermal drug delivery system, made from 2-hydroxyethyl methacrylate (pHEMA) and ethylene glycol dimethacrylate (EGDMA) as well as light-responsive ibuprofen-loaded 3,5-dimethoxybenzene conjugates [217]. The MNs containing the conjugate and drug were inserted into the skin and irradiated with UV light, stimulating cleavage of the conjugate and releasing ibuprofen as the hydrogel of the MN array swelled. This system remained intact in vitro and delivered up to three doses of $50 \mathrm{mg}$ ibuprofen, over a prolonged period (up to $160 \mathrm{~h}$ ) upon application of an optical trigger [217]. The release could also be turned on and off by turning the light on and off, respectively. Consequently, this type of system offers great potential as a stimuli-responsive delivery platform, where "on-demand" drug delivery is required, with triggered sustained-release analgesia as an obvious example. Similarly, it should be possible to design responsive drug delivery systems to facilitate drug 1057 dosing-on-demand to accommodate patient regimens as well as the delivery of multiple dosages at one time. Some photo/radio-sensitive nanoparticle variants possess interesting radiation responsive properties that have been used to design light-responsive drug release from $\mathrm{MN}$ arrays. Chen et al. designed silica-coated $\mathrm{LaB}_{6} @ \mathrm{SiO}_{2}$ nanostructures (serving as nearinfrared radiation (NIR) absorbers), which were subsequently incorporated into biodegradable PCL MNs [182]. This nanoparticle variant mediated a light to heat transduction, thus acting as a local heat source when the array was irradiated with external NIR. Following irradiation, the increased temperature caused a phase transition of the MNs thereby increasing the mobility of the polymer chains, thus leading to the melting of the polycaprolactone to trigger the drug release from the MN array. Rhodamine $6 \mathrm{G}$ was incorporated into MNs as a model molecule to ascertain this remote trigger behaviour. Furthermore, this NIR-light activated device was evaluated in vivo in rat models for the controlled release of DOX [182]. Recently, Liu et al. further explored the use of NIR for on-demand delivery of metformin from dissolving composite MNs coated with lauric acid, containing bismuth (Bi) nanodots stabilised by PVP as the photothermal conversion agent [218]. Upon exposure to NIR irradiation, light-to-heat transduction caused the lauric acid coating to melt, with the subsequent dissolution of the polymer upon contact with the interstitial fluid. Thus, enabling the encapsulated metformin to release into the skin tissue, producing a remarkable sustained hypoglycaemic effect in diabetic rats in vivo [218]. In bio-responsive $\mathrm{MN}$, bio-responsive polymers and micro- or nanoparticles, which are sensitive to physiological signal, are utilised for drug delivery in response to biological stimuli. Bioresponsive MNs have garnered research interest in recent years, particularly concerning glucose-responsive smart insulin delivery for the potential treatment of 
diabetes [219]. Recently, in work conducted by the same Group, Yu et al. further investigated the potential of a removable coin-sized $\left(\sim 5 \mathrm{~cm}^{2}\right) \mathrm{MN}$ patch that released encapsulated insulin cargo in response to hyperglycaemic hypoxia in situ [132]. MNs were loaded with insulin and a non-degradable glucose-responsive polymeric matrix, achieved via in situ photopolymerisation. Under hyperglycaemic conditions, phenylboronic acid units within the polymeric matrix reversibly formed glucose-boronate complexes that, owing to their increased negative charge, induced swelling of the polymeric matrix and thus weakened the electrostatic interactions between the negatively charged insulin and polymers, encouraging the rapid release of insulin. Evaluation of the $\mathrm{MN}$ in insulin-deficient minipigs (>25 $\mathrm{kg}$ ) resulted in extended blood glucose regulation beyond $20 \mathrm{~h}$ from a single $\mathrm{MN}$ patch $\left(\sim 5 \mathrm{~cm}^{2}\right)$. Consequently, MN-mediated responsive drug delivery systems have the potential to combine the essential features of sustained drug delivery in clinical applications.
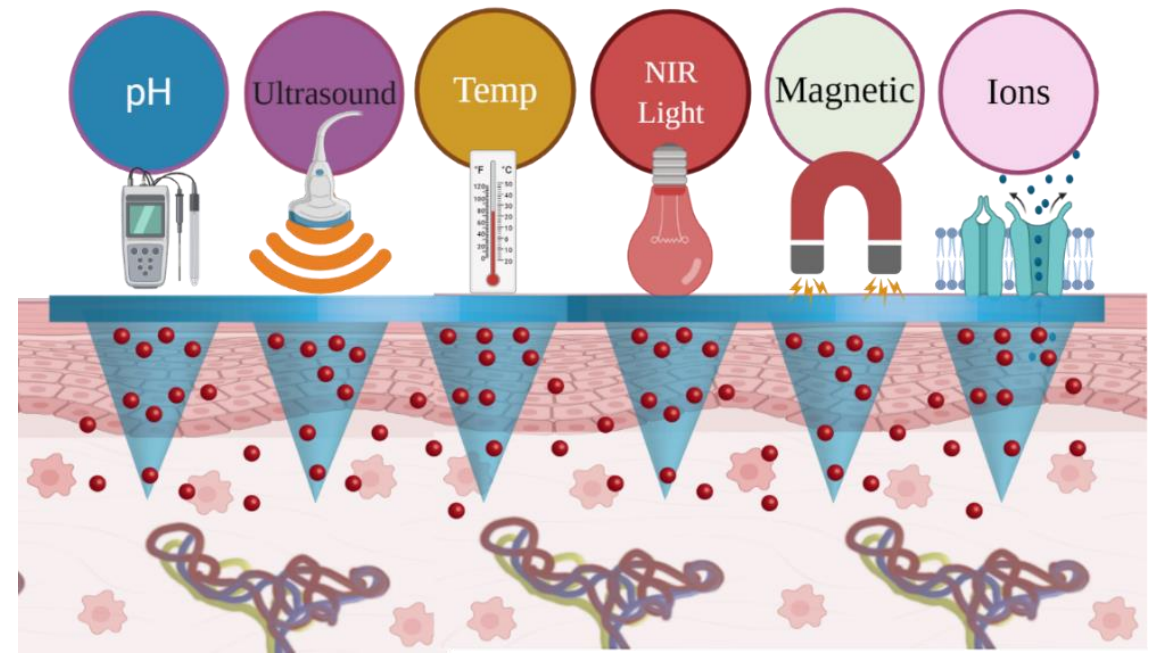

Figure 7. Different type of stimuli-responsive MN

\subsubsection{Hollow MNs}

Hollow MNs are micron dimension needles quite similar to a hypodermic needle in shape. Hollow MNs materialised to inject liquids and suspensions for drug infusion into the skin or ocular tissue through the MN bore. Hollow MN has numerous advantages, for instance, they can be used to inject a wide variety of fluids or particulate system into the skin/ ocular site and offer the highest precision in dose delivery compared to other MN types. Additionally, hollow $\mathrm{MN}$ allows screening the different liquid formulations by simple steps while this is not the case with other types of MN [220]. This type of MN can be used to deliver particulate formulations like nanoparticles or microparticles for controlled/sustained delivery. Hollow MN has been 
developed and investigated for the delivery of varieties of nanoparticles and microparticles by researchers. For the first time, Wang et al. investigated the microinjection of polymeric microparticles (2.5 and $2.8 \mu \mathrm{m})$ using hollow glass MNs. This work assessed the ability to use hollow MN to inject particulate system intradermally. The particles were effectively implanted intradermally into the skin of hairless rats (ex vivo and in vivo) by tailoring the optimal injection pressure [221]. Also, Häfeli et al. designed a silicon hollow MNs device ( $8 \mathrm{MNs}$ ) that attached with flexible PDMS reservoir to inject different aqueous suspension containing fluorescent polystyrene microspheres $(0.7 \mu \mathrm{m}$ in diameter $)$ and blue polystyrene microspheres $(0.93 \mu \mathrm{m}$ in diameter) into the skin up to a depth of $70 \mu \mathrm{m}$ [222]. In another study by Du et al., four types of nanocarriers including PLGA, mesoporous silica, liposomes and, gelatin nanoparticles were used to encapsulate OVA and it's adjuvant and, subsequently, administered intradermally by hollow MNs to assess the different physiochemical properties of these nanoparticles [223]. These reported studies prove that it is possible to deposit the nano/micro-particulate system for further sustained release application for varieties of chronic disease conditions.

Apart from the intradermal application, ocular drug delivery via hollow $\mathrm{MN}$ is also a promising research area, especially, when administering drugs to the back of the eye for sustained therapeutic effect. Hollow MN arrays in combination with micro/nano-carriers can play an interesting role in this niche area for long-acting sustained delivery. Jiang et al. successfully demonstrated the ability of hollow glass MN to infuse nano- and micro-particles into the sclera in a minimally invasive manner. Nile red encapsulated PLA nanoparticles were easily injected using an insertion-retraction method. However, latex fluorescent microparticles only injected in presence of hyaluronidase and collagenase spreading enzymes to disrupt scleral structure [224]. A similar hollow MN design was employed by Patel et al. to inject various different sizes of nanoparticles ( $20 \mathrm{~nm}$ to $1000 \mathrm{~nm}$ ) to the interior suprachoroidal space of the whole eye (rabbit, pig and human) ex vivo with a different needle length and infusion pressure. Results demonstrated that particle size, MN length and, pressure played an important role in effective delivery into the suprachoroidal space [225]. Kim et al. demonstrated the use of hollow MNs to administer a core of high-density perfluorodecalin ( $\leq 35 \mu \mathrm{m}$ in diameter) particles surrounded and stabilised by fluorescein-tagged, polystyrene nanoparticles (PED) to the back of the eye of rabbits in vivo. After infusion of this high-density PED emulsion into the suprachoroidal space of the eye, at least $50 \%$ of the injected nanoparticles were detected to be near the macula or ciliary body depending on the eye cornea orientation (gravity-mediated targeting) after injection and 5 days later [226]. 
1136 The therapy of diseases such as diabetes, cancers, cardiovascular, neurological, chronic 1137 infectious and, musculoskeletal system diseases, can be significantly improved by long-acting 1138 formulations. Long-acting implantable formulations are also useful for contraception and 1139 vaccination. Long-acting MN formulations can be developed for controlled drug delivery to 1140 improve therapeutic effects, decrease dosing frequency, and prevent potential toxicity. Many therapeutic agents, such as biomacromolecules (e.g. peptides, proteins and gene therapeutics), drugs with low bioavailability and, drugs for local delivery are ideal candidates for creating long-acting micro-implants. However, not all drug molecules are suitable for these

1144 formulations. The ideal formulation should deliver the drug to the site of action at the correct 1145 therapeutic concentration for as long as the treatment is desired. The therapeutic and the 1146 physicochemical properties of a drug determine the preferred route of administration (oral or parenteral) as well as the most appropriate delivery systems [227]. This section briefly outlines examples of disease or drugs classifications that are suitable for long-acting sustained delivery by MN delivery.
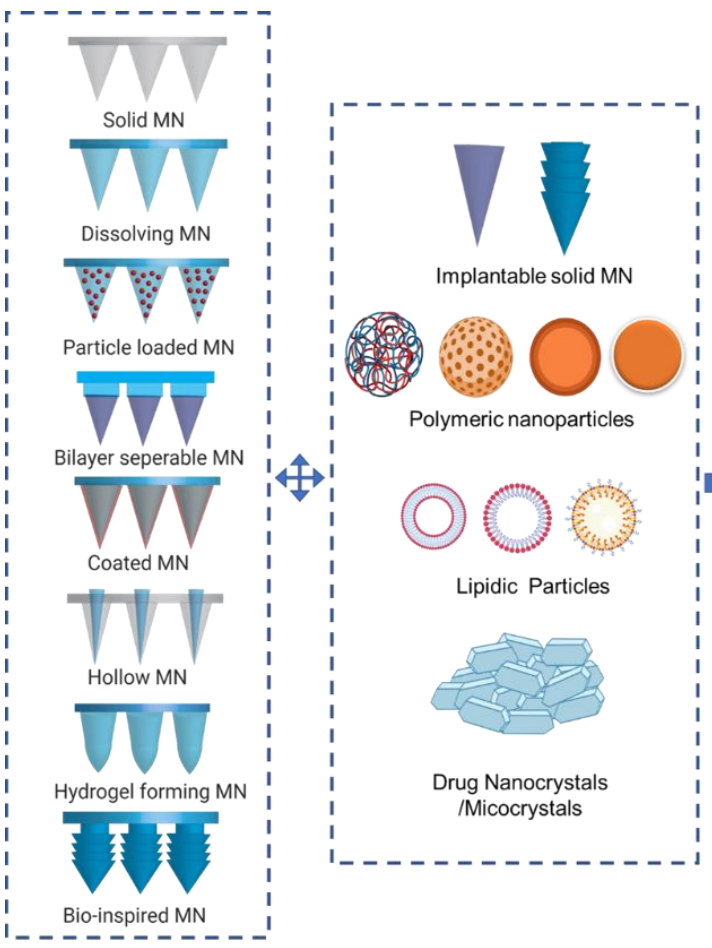

$$
\begin{array}{|c|}
\hline \text { Skin ailments } \\
\hline \text { Lymphatic targeting } \\
\hline \text { Infectious diseases } \\
\hline \text { Vaccine delivery } \\
\hline \text { Gene Therapeutics } \\
\hline \text { Contraceptives } \\
\hline \text { Neurological disorders } \\
\hline \hline \text { Diabetes/Insulin } \\
\hline \hline \text { Cancer } \\
\hline \hline \text { Cardiovascular diseases } \\
\hline \hline \text { Ocular diseases } \\
\hline
\end{array}
$$

1151 Figure 8. Schematic representation of combination approaches with MN and Nano/microformuations for longacting drug delivery for potential disease conditions 
Protein/peptide therapeutics has been utilised in the treatment of many diseases in recent years and have received major research interest as a result. The main route of drug delivery for peptide and protein therapeutics is either through subcutaneous, IM or IV injections [228]. However, these routes suffer from proteolytic degradation and antibody neutralisation, which in turn, leads to short plasma half-lives. Therefore, this results in the necessity of repeated injection of the drug, albeit this comes at the cost of patient adherence which influences their quality of life [229]. To overcome the above limitations, long-acting injections or implants have been developed for such products. Examples include in situ injectable depot-forming systems, microspheres, biodegradable and non-biodegradable polymeric implants, implantable pump systems and, drug-eluting stents [230]. Minimally invasive peptide and protein delivery technologies are promising for product differentiation and value creation for industry, particularly for their potential in vaccination. In the last two decades, MN patches have made substantial medical advances in systemic delivery of potent biotherapeutics and vaccines [231]. MN delivery of genetic materials, in particular plasmid DNA (pDNA), to the skin represents a potential new method for the clinical management of genetic skin diseases and cutaneous cancers, and intradermal genetic immunisation [169].

\subsubsection{Vaccine}

Most vaccines are administered by the subcutaneous or IM routes using a needle and syringe; and to a lesser extent the ID route, which is used for the administration of Bacillus CalmetteGuérin (BCG) and rabies vaccines. However, there has been renewed interest in ID vaccine delivery in the last few decades. This is owing to the fact that the ID space of human skin, unlike that of the muscle and subcutaneous space, is a rich network of potent antigen-presenting cells (APCs) including Langerhans Cells (LCs) and many sub-sets of dermal dendritic cells (dDCs) with roles in both innate and adaptive immunity. Thus, suggesting that the delivery of vaccines to ID layers should be more effective with smaller amounts of vaccine antigen [232]. Another tactic to increase the immune response from vaccination is extending the antigen presentation, particularly for the inactivate/subunit vaccines that usually require one or more booster administration separated by months or years to induce lasting immunity. Long-acting extended delivery of an inactivated antigen could present the specific antigen over a prolonged period, thereby generating a stronger immune response. A new add-on for long-acting extended delivery of vaccine can improve immune responses by ID route as described previously [233]. ID injections are typically performed using the Mantoux technique, first described in 1910 by French physician Charles Mantoux for ID injection of tuberculin as a diagnostic tool for 
screening of tuberculosis, in which a small 28 gauge needle is injected at a close angle (10$15^{\circ}$ ) compared to that subcutaneous $\left(45^{\circ}\right)$ injections [234]. As such, administration of ID injections is more complicated than conventional injection techniques, and, therefore, it requires special training to target the skin reliably [235]. Further issues arise with the potential of ID injection, which has been associated with adverse events such as pain, inflammatory changes and the development of abscesses. Considering all the issues with other routes and ID administration of vaccine by syringe, the concept of MNs has emerged as a technological solution for ID administration of vaccines [6,236,237]. Recently, MN technology has applied for safe and effective ID delivery of vaccines through the painless mechanical disruption of the $S C$ [238]. MNs are arrays mechanically pierce the skin barrier, $S C$, to deposit vaccines in the ID space. MNs have been demonstrated as a potential strategy for pain-free vaccine administration in early clinical trials [239-241] These MN patches are a currently evolving technology to control vaccine kinetics.

As highlighted in Figure 8, the potential for successful MN assisted intradermal delivery of vaccine/immunotherapeutics formulation when combined with various nanotechnology approaches has been illustrated. Joyce et al. demonstrated for the first time the effect of different extended-delivery profiles on immune response using three different licensed vaccines (inactivated polio vaccine, tetanus toxoid and influenza) administered to the ID space of skin. The authors investigated the extended-delivery profiles by administering multiple fractional vaccine doses by ID injection or MN patch. Within the study, single doses of extended-delivery vaccination were compared to two-dose bolus vaccination. It was demonstrated that extended-delivery for one month further improves immune response compared to delivery over shorter times. Interestingly, the extended-delivery achieved through repeated $\mathrm{MN}$ patch vaccination further increases the immune response compared to injections [233]. This detailed proof of concept study investigating different vaccines approaches opened further research avenues to optimise the $\mathrm{MN}$ formulation, to achieve the long-acting extended profile after single MN application. Extended delivery of vaccines to the skin not only improves immune response but also has the potential for dose sparing and reducing the number of vaccine doses required to reach protective immune levels.

Boopathy et al. engineered implantable solid pyramidal MN arrays with silk fibroin protein tips with encapsulated a stabilised HIV envelope trimer immunogen and adjuvant, supported on a dissolving polymer base for sustained-release therapy. In vitro release studies demonstrated that the structural integrity of the antigen was preserved and, a molecular weight- 
dependent release over time from the MNs. Briefly, upon MN application to the skin, within 5 minutes the vaccine-loaded silk tips were implanted in the deep ID space, from where they released vaccine over a period of time controlled by the crystallinity of the silk matrix. In vivo studies in mice, demonstrated that the subunit vaccine was released over 2 weeks in the skin, correlating with increased germinal center (GC) B cell responses, an approximate 1,300-fold increase in serum IgG titers and a 16-fold increase in bone marrow (BM) plasma cells compared with bolus immunisation. This early study proved that implantable MNs have the practical potential to substantially enhance humoral immunity to subunit vaccines [242].

Du et al. investigated hollow $\mathrm{MN}$-assisted intradermal delivery of nanoparticulate vaccines to modulate the immune response of protein antigen. Within this study, four different types of nanoparticles were investigated for their effect upon kinetic release parameters, namely; PLGA nanoparticles liposomes, mesoporous silica nanoparticles (MSNs) and gelatin nanoparticles (GNPs). These different nanoparticles also used for comparison between those a model antigen ovalbumin (OVA) with and without an adjuvant poly(I:C). Hollow-MN applicator was also designed to inject nanoparticle suspensions precisely into murine skin at a depth of about 120 $\mu \mathrm{m}$. Interestingly, OVA/poly(I:C)-loaded nanoparticles and OVA/poly(I:C) solution elicited equally robust total $\mathrm{IgG}$ and $\mathrm{IgG1}$ immune responses. However, the co-encapsulation of OVA and poly(I:C) in nanoparticles significantly augmented the $\mathrm{IgG} 2 \mathrm{a}$ response compared to OVA/poly(I:C) solution [223]. PLGA nanoparticles and liposomes induced stronger IgG2a responses than MSNs and GNPs, that proves the direct effect of small particle size and sustained release effect from the PLGA nanoparticles and liposomes for the antigen and adjuvant. In the case of cellular responses, the highest $\mathrm{CD}^{+}$and $\mathrm{CD} 4^{+} \mathrm{T}$ cell responses were induced by liposomes. This study demonstrated that the applicator controlled hollow MN delivery may be a potential choice for effective intradermal injection of long-acting nanoparticle vaccines for extended humoral and cellular immune responses [223].

With particular and recent significance, coronaviruses create a grave threat to global health as shown by Middle East Respiratory Syndrome (MERS), Severe Acute Respiratory Syndrome (SARS) and, COVID-19. SARS Coronavirus (SARS-CoV), MERS Coronavirus (MERS$\mathrm{CoV}$ ), and the novel coronavirus, now officially named SARS-CoV-2, are the contributing sources of the SARS, MERS, and COVID-19 disease outbreaks, respectively. The global pandemic caused by COVID-19 has emerged as a severe public health issue crippling world health care systems [243,244]. Reliable vaccines that swiftly induce potent and long-lasting virus-specific immune responses against these coronaviruses urgently required. Kim et al. 
1253

1254

1255

1256

1257

1258

1259

1260

1261

1262

1263

1264

1265

1266

1267

1268

1269

1270

1271

1272

1273

1274

1275

1276

1277

1278

1279

1280

1281

presented the rapid design and translational development of dissolving MN-based SARS-CoV2 recombinant vaccines. Initially, they tested the pre-clinical immunogenicity of MERS-CoV vaccines in mice. The vaccine was delivered $S C$ by traditional injection, or intracutaneously by dissolving MN to evaluate the virus-specific IgG antibodies in the mice serum. Specifically, dissolving MN delivered MERS-S1 subunit vaccines elicited strong and long-lasting antigenspecific antibody responses. Based on this, the authors also tested SARS-CoV-2 subunit vaccine loaded dissolving MN in mice. Most importantly, these MN delivered SARS-CoV-2 S1 subunit vaccines elicited potent antigen-specific antibody responses that were evident starting 2 weeks after immunisation. They anticipate clinical development of these dissolving MN delivered recombinant protein subunit vaccines against SARS, MERS and other emerging infectious diseases [245]. This MN-assisted vaccination could significantly speed up roll-out and distribution of such vaccines without stringent storage condition as well as lowering the burden on the global healthcare system.

Recently, the MN-mediated delivery of virus-like particles (VLP) vaccines has gathered greater interest. VLP is self-assembly particles $(30-90 \mathrm{~nm})$ self-possessed of viral structural proteins without a nucleic acid genome or a lipid envelope. Initial studies performed by Kang's group were conducted using coated MN patches with viral particles. For the first time, the ID route was utilised to immunise mice and effectively demonstrated that the size of antigens encapsulated in MN patches had no detrimental impact on the immune efficacy in mice. Moreover, the MN patch induced a significant long-term protective immune response with a high level of IgG in serum and IgA in lung alveolar fluid [246]. In further studies, MNs were utilised to deliver the commercially approved VLP vaccine Gardasil (composed of the L1 VLP capsid of human papillomavirus), for prophylaxis of human cervical cancer. To assess vaccine immunogenicity, Corbett et al. applied a novel dry vaccine coated densely packed MN patch $\left(\right.$ Nanopatch $\left.^{\mathrm{TM}}\right)$ to $\mathrm{C} 57 \mathrm{BL} / 6$ mice and compared the response to the intramuscular injection control. The dose delivered by the MN array was sufficient to produce a maximal virus neutralising serum antibody at day 28 post-vaccination and, a sustained response for 16 weeks, with higher titres than intramuscular injection delivery at equivalent doses of VLP vaccine [247]. 


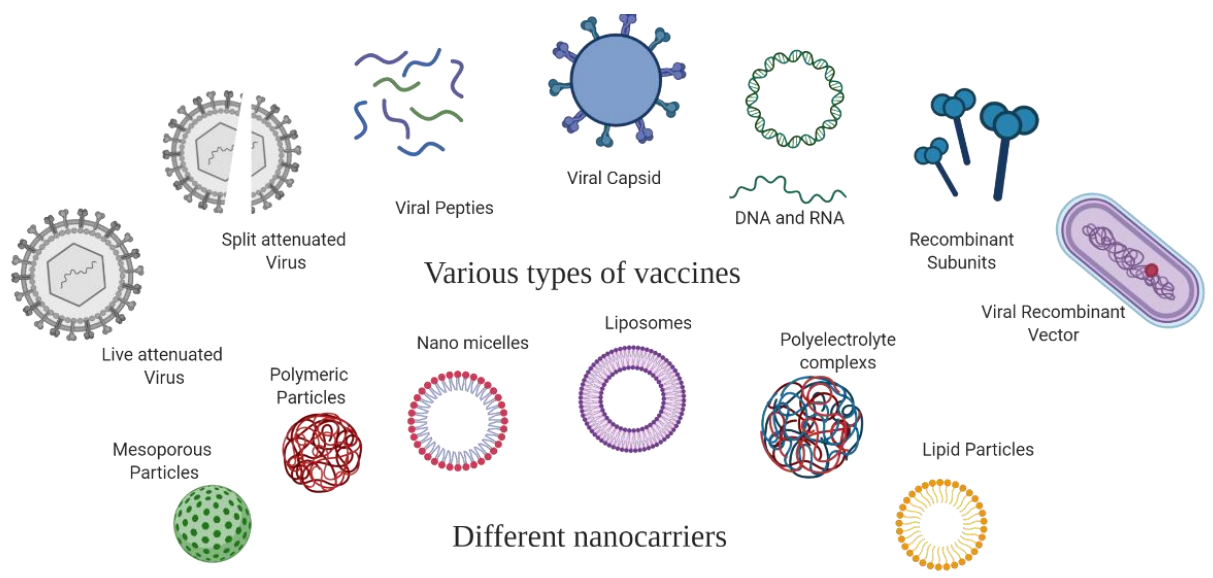

Figure 9. Intradermal prolonged delivery strategies of various vaccines/immunotherapeutic formulation by MN

\subsubsection{Gene/immunotherapy}

1285 Gene/immunotherapy offers great promise to treat a variety of skin (e.g., skin cancers, abnormal scar, psoriasis, hyperpigmentation wounds and infections) and systemic (cancer) diseases [248,249]. Genetic materials can be administered into skin after being coated on the surface of the solid MNs, embedded within the degradable/porous polymeric MNs, injected through hollow MNs, or smeared onto MN-treated skin with the aid of electroporation [250].

Qu et al. recently developed a gelatin methacryloyl (GelMA) MN for local and sustained delivery of pDNA to meet the increasing need for ID gene therapy. Within the study, Poly $(\beta-$ amino ester) (PBAE) nanoparticles were utilised to achieve the intracellular delivery and high local transfection efficiency of plasmid DNA encoding EGFP (pEGFP) as the model cargo both in vitro and in vivo. After being embedded in the GelMA MNs, sustained release of DNAencapsulated PBAE nanoparticles was attained and the release kinetics was found to be controlled by altering the degree of crosslinking of the GelMA hydrogel matrix. Here, The GelMA MN matrix provided protection for the encapsulated PBAE/DNA nanoparticles and, photocrosslinkability of GelMA was found to control both mechanical strength and DNA 
release profiles. The authors used a C57B1/6 mouse model to validate the in vivo gene delivery efficacy of the MN/PBAE/DNA. Mice skin harvested after 3 days of $\mathrm{MN}$ application to confirm the pEGFP delivery and local inflammatory reactions. MN/PBAE/ DNA enabled the transfection rate of cells in the region of interest was found to be $\sim 31 \%$ without causing significant skin damage. These preclinical findings opened up the new avenue for localised targeted and sustained delivery of genetic materials through ID route [251].

The purpose of cancer immunotherapy is to generate a systemic immune response by the host to eradicate the tumour cells [252]. Considering the immunological advantages of skin and pain-free delivery of MN, the delivery of cancer immunotherapy has been explored utilising the application of MN patches, containing DNA/RNA, immunostimulatory adjuvants and/or antigens, as anticancer therapeutic approaches [253]. Recently, Zaric et al. reported the potential of dissolving PMVE/MA MN arrays laden with nanoencapsulated model antigen (OVA) to increase vaccine immunogenicity by targeting the antigen specifically to contiguous DC networks within the viable dermis. Following in situ uptake, skin-resident DCs, delivered antigen-encapsulated PLGA nanocarriers to cutaneous lymph nodes where they subsequently induced substantial expansion of antigen-specific $\mathrm{T}$ cells. Moreover, in vivo studies in mice models demonstrated that antigen-encapsulated nanoparticle vaccination via $\mathrm{MN}$ generated robust antigen-specific cellular immune responses, due to sustained antigen delivery from the vaccination site as a result of nanoencapsulation facilitating antigen retention in the skin layers. Furthermore, this immune system activation, mediated by the slow OVA nanoparticles release from the $\mathrm{MN}$, prevented the development of ovalbumin expressing B16 melanoma tumours for 13 days. This approach was found to provide complete protection in vivo against both the development of antigen-expressing B16 melanoma tumours and a murine model of parainfluenza, concluded the stimulation of antigen-specific cytotoxic $\mathrm{CD} 8^{+} \mathrm{T}$ cells that stemmed inefficient clearance of tumours and virus, respectively. Furthermore, nanoencapsulation provided enhanced antigen stability in MNs. Therefore, the use of biodegradable polymeric nanocarriers for selective targeting of antigen to skin DC subsets through dissolvable MNs presents a promising technology for improved immunotherapy efficacy, adherence, and coverage [254]. Wang et al. reported another approach to overcome these challenges by nanoparticle-embedded MN system that contains a dissolvable HA matrix and mesoporous silica-coated upconversion nanoparticles (UCNPs@mSiO2). The mesoporous silica (mSiO2) shell acts as the reservoir to protect siRNA and cellular uptake enhancer of siRNA. At the same time, the upconversion nanoparticle (UCNP) core allows the live tracking of MN skin 
penetration and NP diffusion through upconversion luminescence or optical coherence tomography imaging. This proof of concept multifunctional MN system was used to deliver molecular beacons (MBs) and siRNA targeting transforming growth factor-beta type I receptor (TGF- $\beta$ RI) that may be potentially used for abnormal scar treatment [255].

Qu et al. recently developed a gelatin methacryloyl (GelMA) MN for local and sustained delivery of pDNA to meet the increasing need for ID gene therapy. Within the study, Poly( $\beta$ amino ester) (PBAE) nanoparticles were utilised to achieve the intracellular delivery and high local transfection efficiency of plasmid DNA encoding EGFP (pEGFP) as the model cargo both in vitro and in vivo. After being embedded in the GelMA MNs, sustained release of DNAencapsulated PBAE nanoparticles was attained and the release kinetics was found to be controlled by altering the degree of crosslinking of the GelMA hydrogel matrix. Here, The GelMA MN matrix protected the encapsulated PBAE/DNA nanoparticles and, photocrosslinkability of GelMA was found to control both mechanical strength and DNA release profiles. The authors used a C57B1/6 mouse model to validate the in vivo gene delivery efficacy of the MN/PBAE/DNA. Mice skin harvested after 3 days of $\mathrm{MN}$ application to confirm the pEGFP delivery and local inflammatory reactions. MN/PBAE/ DNA enabled transfection rate of cells in the region of interest was found to be $\sim 31 \%$ without causing significant skin damage. These preclinical findings opened up the new avenue for localised targeted and sustained delivery of genetic materials through ID route [256]. Duong et al. recently developed DMN-based polypeptide cocktails for the triggered delivery of ovalbumin-expressing plasmid OVA (pOVA) and immunostimulant, polyinosinic:polycytidylic acid (poly(I:C)) to augment cancer immunotherapy. To enhance the cellular uptake to APCs, pOVA and poly(I:C) were loaded into a nanosized polyplex prepared using cationic amphiphilic conjugates. MNs were fabricated from biocompatible and hydrophilic copolymers consisting of a cationic polypeptide and Poly (ethylene glycol) (PEG) as a matrix. The cationic nanopolyplex effectually transfected pOVA and poly(I:C) into the intracellular compartments of macrophages and dendritic cells. MN assisted cutaneous administration of cocktails in mouse model elicits a stronger antigenspecific antibody response than subcutaneous administration of the MN-free nanopolyplex. The MN cocktail therapy synergistically augmented the therapeutic effect in B16/OVA melanoma tumours [257]. In final remarks, the further development of MNs for gene therapy depends not only on the improved fabrication of MNs but also on the combined use of different delivery techniques (adjuvants, nanocarriers, targeting moieties) to achieve optimal and long lasting therapeutic outcomes [256]. 
1366

1367

1368

1369

1370

1371

1372

1373

1374

1375

1376

1377

1378

1379

1380

1381

1382

1383

1384

1385

1386

1387

1388

1389

1390

1391

1392

1393

1394

1395

1396

1397

The dermis is very well perfused with dense vascular and lymph capillary networks. In addition, the local capillaries and lymph vessels have less barrier properties to absorption owing to their reduced wall thickness and endothelial barrier. Lymphatic vessels situated in the dermis absorb and transfer interstitial fluid and administered macromolecules from the skin to bloodstream. Of particular importance, the lymphatic system with secondary lymphoid organs, mainly lymph nodes (LNs) play an essential role in absorbing several antigens and modulating the immune response via prophylactic or therapeutic interventions [258]. Skinassociated lymphoid tissue plays a vital role in vaccine delivery, due to the richness of immune cells, including dermal dendritic cells, $\mathrm{T}$ cells and macrophages. Therefore, the intradermal route of vaccination, particularly in use with $\mathrm{MN}$, has shown prolonged and enhanced immune response compared with the IM route, allowing for an effective reduction in dose with long term protection [259].

Niu et al. reported a study where model antigens encapsulated PLGA nanoparticles (less than $100 \mathrm{~nm}$ ) administered via hollow MN array for intradermal delivery in rats. Compared to IV and subcutaneous routes, intradermal delivery of polymeric nanoparticles via a hollow MN array resulted in an early burst transit through the draining lymph nodes and a relatively limited overall systemic exposure. Moreover, antigen-loaded NPs delivered by hollow MN array elicited a significantly higher $\operatorname{IgG} 2 \mathrm{a}$ antibody response and a higher number of interferon (IFN)- $\gamma$ secreting lymphocytes in comparison to antigen-loaded NPs given by IM injection and soluble antigen delivered through hollow MN array. Antigen-loaded NPs provoked better antibody response than the soluble antigen formulation because of the sustained release of the antigen at tissue level (depot in skin/draining lymph nodes), at the cellular level (nanoparticles internalised by $\mathrm{dDCs}$ ), and at organelle level (nanoparticles in endolysosome/cytosol). This unique lymphatic targeted and sustained release property of antigen-loaded nanoparticles formulation via MNs might also apply to biologic drug development [260]. Previous studies using fluorescence-labelled dextran and liposomes verified the optimal size and charge characteristics for lymphatic delivery [261,262]. Nanoparticle uptake from the lymph bed capillaries, nanoparticles with sizes amongst $10 \mathrm{~nm}$ and $100 \mathrm{~nm}$ are held up by the lymphatic capillaries. In comparison, particles of less than $10 \mathrm{~nm}$ are absorbed by blood capillaries, and particles of larger than $100 \mathrm{~nm}$ are retained at the administration site [263]. Targeted delivery of therapeutics can be achieved utilising carriers with a specified affinity to the target tissue or directly administering the target organ [264]. 
As there is a vast abundance of lymphatic vessels situated in the intradermal space of the skin, it is the easiest way to access the potential of lymphatic targeting for a potential cure and prophylactic of infectious diseases. Several recent studies have explored minimally invasive MN devices as novel trans- and intra-dermal drug for lymphatic targeting. These studies have successfully shown increases in drug concentration in the lymph nodes compared to other injectable routes. Harvey et al., for the first time demonstrated by the NIR imaging studies with Indocyanine Green (ICG) dye, that lymphatic absorption may be a significant contributor of uptake for biomolecules from MN delivery [4]. Additionally, our Research Group has previously developed $\mathrm{MN}$ formulations in combination with a long-acting rilpivirine nanosuspension to be delivered intradermally, with detectable levels of rilpivirine in the lymph nodes of treated rats, justifying the continued exploration of this approach [118]. Further to this, we recently explored the incorporation of novel lipid nanoparticles of multiple antifilariasis drugs (doxycycline, diethylcarbamazine and albendazole) into dissolving $\mathrm{MN}$ for intradermal delivery, to evaluate the effect upon lymphatic uptake. The SLNs were prepared from Geleol ${ }^{\circledR}$ as a lipid matrix and Tween 80 as a stabiliser and with sizes of $<100 \mathrm{~nm}$. In vitro drug release was shown to be sustained for a longer duration over $48 \mathrm{~h}$ from SLNs, compared to pure drugs counterparts. The SLNs were mixed into a polymeric hydrogel, which was then cast to form SLNs-loaded MNs and, all three separate MN systems exhibited sufficient mechanical and insertion properties. Dermatokinetic studies further demonstrated that greater than $40 \%$ of the drug was deposited in the dermis of excised neonatal porcine skin for up to 24 $\mathrm{h}$ post-MN application, indicating the high possibility of the SLNs to be taken by the lymphatic system with longer duration of a slower release. In in vivo studies in rats, a 4 to 7 -fold higher lymphatic concentration of each of the three drugs was achieved following intradermal delivery compared to oral administration. This way, targeting the drug to the lymph nodes, where filarial nematodes reside in diseased patients, prominent to an effective therapy for lymphatic filariasis $[5]$.

\subsection{HIV infection}

Poor adherence to oral antiretroviral therapy (ART) remains an important challenge in the treatment of HIV. Moffatt et al. recently investigated the opinions from various end-users surrounding the translation of MN technology to general clinical practice, with a particular focus on the delivery of antiretroviral therapy. The consensus surrounding MN technology from patients with HIV involved in qualitative focus groups was found to be positive, with patients highlighting benefits pertinent to HIV, including discreet self-application and potential 
sustained release thus avoiding daily pill taking and the associated side effects. Patient concerns focused on the need for varied $\mathrm{MN}$ dosing schedules and a reluctance to change from established antiretroviral therapy, which should act as a motivator for those involved in MN development to ensure a patient-centred $\mathrm{MN}$ product is delivered. The findings of this preliminary study provided an initial indication of MN acceptability and support of their clinical translation particularly for use within HIV, and, thus, highlighted that there was a compelling need for an alternative minimally invasive, long-acting method of antiretroviral delivery, to that of current oral antiretroviral therapy [265]. This work has been pioneered by Donnelly and co-workers. For example, McCrudden et al. developed dissolving polymeric MNs for the sustained delivery of rilpivirine to improve the adherence to prevention and treatment regimens of HIV. The MNs were fabricated using PVA polymer and an industrially manufactured long-acting rilpivirine nanosuspension that is currently under clinical development as an injectable, attached to a pre-formed bioadhesive baseplate. By conducting in vivo investigation in rats, the authors showed the comparable distribution of rilpivirine in plasma (extended for 56 days), localized tissue, and lymphoid tissue between nanosuspension delivery via MNs and intramuscular injections. Based on the results obtained from this study, a cautious extrapolation estimated that a patch size of approximately $28 \mathrm{~cm}^{2}$ could be sufficient to maintain therapeutic plasma levels of rilpivirine in humans over 7 days [164]. Subsequent work from the same Research Group has led to the development of similar MNs for the intravaginal delivery of rilpivirine in a discrete manner. Encouragingly, positive results comparable to intramuscular injection were once again achieved [164].

In a further study from the same Research Group, Tekko et al. produced novel bilayer dissolving polymeric MNs with high drug loading (60\%) for the sustained delivery of another ART drug- cabotegravir to improve the adherence to prevention regimen of HIV [266]. The MNs were fabricated using a blend of PVA/PVP and an industrially manufactured long-acting cabotegravir nanosuspension, attached to a drug-free PVA/PVP-based baseplate. The fabricated MNs sufficiently strong to pierce Parafilm $\mathrm{M}^{\circledR}$ and excised porcine skin models and could withstand up to $32 \mathrm{~N}$ of applied compression force. The in vivo study demonstrated that MNs was able to deliver a substantial amount of the loaded dose and maintained drug plasma concentrations above the human therapeutic concentrations for PrEP (Pre-exposure prophylaxis) of 4x P-IC90 (664 ng/ml) over 28 days study period. These studies again suggest that MNs could be also used to deliver the ART drugs in a sustained manner over prolonged, 
thus demonstrating early promise for the future of $\mathrm{MN}$-mediated delivery in the treatment of HIV, and supports the further investigation of this platform in delivering other ART candidates.

\subsection{Contraceptives}

To address the unmet worldwide need for family planning, scientists have been compelled to re-explore several approaches of modern contraception, focusing on those that are long-acting, reversible and, provide flexibility to a woman [267]. Most of the water-insoluble progestin based contraceptive progestogens are generally formulated as an oral pill, injection, implant, or vaginal ring. The last 3 dosage forms can deliver progesterones in a sustained release manner. However, either injecting/implanting or surgical removal of implants will raise the burden of treatment to a patient. As such, there is a strong need for long-acting contraceptives that can increase contraceptive accessibility and convenience for women. MNs are considered minimally invasive devices that can disrupt the $S C$ barrier to achieve transdermal drug delivery and improve drug efficiency. There has been good progress toward the long-acting MN research for contraceptive agents in the last five years [268].

Prausnitz et al. published work with effervescence-triggered rapidly separating LVN loaded PLGA MNs. MN tips separated from the patch baseplate and implanted within the skin due to effervescence that was triggered upon contact with skin's interstitial fluid, as demonstrated in both rats and human participants. In rats, MN-mediated delivery of LVN exhibited sustained release above the relative human therapeutic plasma levels required to maintain adequate contraceptive control for more than 1 month. A placebo MN patch was well-accepted in human participants without any skin or allergic reactions. Women of reproductive age in three world regions expressed interest in and, a preference for long-acting contraception by $\mathrm{MN}$ patch compared to other available options. These findings signify that an effervescent MN patch could enable greater access to long-acting contraception [47]. He et al. reported novel longacting MNs that can be inserted into the skin in situ by a relatively simple and rapid application. MNs were fabricated to entrap etonogestrel in the PLGA tips by a controllable casting-mould technique, thus avoiding any adverse effect of high temperature on the drug stability. A new formulation method of MNs has used the N-methyl pyrrolidinone as a solvent for the needle tip matrix. Following solidification at $70^{\circ} \mathrm{C}$ for $4 \mathrm{~h}$, the needle tips were adequately strong to puncture the skin. Etonogestrel was shown to crystallise in needle tips uniformly, visualised by a polarising microscope. The ID insertion ratio of the MNs was influenced by the parameters of needle spacing and needle shape. Following optimisation of $\mathrm{MN}$ formulations, the drug 
loading capacity was $153.0 \pm 13.5 \mu \mathrm{g}$, and the drug utilisation rate was up to $92.6 \pm 8.1 \%$. In rats, the pharmacokinetic study of the implantable MNs showed that the plasma etonogestrel level could be detectable until $336 \mathrm{~h}$ and, the AUC0/48h only accounted for $37.8 \%$ of AUC0/ $\infty$ [116]. In additional studies conducted by He et al. investigating the potential sustained release of etonogestrel, dissolving MNs were fabricated with etonogestrel microcrystal particles incorporated for intradermal delivery over 1 week. In vitro release analysis showed that etonogestrel in the microparticles form could produce a greater sustained drug release in comparison to the nanocrystal form. Hydroxypropyl methylcellulose and PVA were employed to fabricate the fast-dissolving needle tips with $50 \%$ drug and drug-free flexible back layer, respectively. The tips of dissolving MNs dissolved in rat skin within $1 \mathrm{~h}$ with etonogestrel deposition efficiency of $63.8 \pm 2.0 \%$. In rats, $\mathrm{MN}$ treatment demonstrated that the plasma levels of etonogestrel were a dose-dependent profile and were much steadier than ID injection. There was no statistical variation between the bioavailability of etonogestrel treated with dissolving MNs or ID injections [269]. Therefore, the MNs with contraceptive drugs (drug crystals, encapsulated in biodegradable particles, or PLGA/PLA tips) provided a perspective with selfadministration features for intradermal sustained delivery [45,47,116,269]. This MN assisted long-acting delivery could offer practical choices for women to have access to safeguard against unwanted pregnancy.

\subsection{Neurological disorders}

\subsubsection{Anti-psychotics}

Treatment of schizophrenia and other psychosis, such as bipolar disorder, is currently supported by a range of antipsychotic drugs, however, their beneficial effect is often limited by the common experience of side effects, resulting in poor adherence to therapy [270]. There is a compelling need for a minimally invasive method of sustained administration of antipsychotics. However, to meet this need further advances in delivery technologies and assessment of these in clinical studies are required in order to broaden the clinical use of antipsychotic formulations for the transdermal treatment of psychiatric illness. In particular, employment of different strategies, such as the use of nanoparticles/vesicles in combination with permeation enhancers or MNs with or without iontophoresis may improve the absorption of antipsychotic drugs through the skin [271]. Moreover, several clinical trials regarding MNs have been conducted, or are ongoing, including studies with drugs acting on the central nervous system (zolmitriptan) [272]. For this reason, looking forward, MNs could represent an 
attractive and valid option to improve antipsychotic drug delivery through the skin. In support of this proposition, dissolving MNs have previously demonstrated the ability to achieved high dose delivery of a hydrophobic nanosuspension in rat models, affording a sustained release of drug from over time the application site [118]. Thus, based on this study, it could be viewed that the dissolving MN intradermally deposits the drug nanosuspension, thus creating a microdepot of the drug in the viable skin layers, from which allows prolonged drug administration. Subsequently, the incorporation of an antipsychotic drug nanosuspension, such as that of aripiprazole or paliperidone, within a dissolving MN system may be a possibility. Thus, upon application to the skin, the antipsychotic drug may slowly release over long periods of time to maintain constant plasma levels, thus affording a minimally invasive alternative method of sustained delivery. This novel dual-concept approach may, in theory, potentially circumvent limitations associated with parenteral and transdermal delivery at the same time. Notwithstanding, MN-mediated delivery of an antipsychotic agent, namely olanzapine, has been recently demonstrated ex vivo [65]. Kearney et al. demonstrated the enhanced release of olanzapine co-solvent loaded reservoirs in combination with hydrogel-forming $\mathrm{MNs}$ in a Franz-diffusion setup, with approximately $50 \%$ of olanzapine delivered at the $24 \mathrm{~h}$ from each of the two reservoir designs evaluated, thus indicative of a sustained release. This work provided early crucial proof-of-concept evidence that MNs are potentially a feasible alternative method to facilitate the delivery of hydrophobic antipsychotic agents. With further evaluation in future clinical studies, this delivery concept could potentially benefit not only therapeutic outcomes but also positively impact patient acceptance of therapy.

\subsubsection{Alzheimer's disease}

Alzheimer's disease (AD) is a progressive neurodegenerative disorder of the central nervous system (CNS), in which there is a continual deterioration of intellectual and social functions, memory loss, personality changes and an inability for self-care [273]. Despite the broad appeal of skin route of administration, rivastigmine $\left(\right.$ Exelon $\left.^{\circledR}\right)$ is the first and only transdermal patch marketed to treat all stages of AD [274]. While commercially successful, it still requires daily administration, and subsequent efforts to increase application time have been investigated, including completion of Phase I trials for the once-weekly donepezil transdermal patch [275], with further studies, are ongoing, such as of the recent advancements made in the 6-day dosage form of transdermal rivastigmine [276]. Sadeghi et al. in this study incorporated rivastigmine within chitosan microparticles further integrated into the patch acrylic adhesive, which provided sustained release of the active drug over almost 1 week in vitro. Subsequently, 
demonstrating a promising feasible replacement from a 1-day dose to 6-day dose application of rivastigmine. While an opportunity presents in this instance to further enhance permeation and sustained release of nanoparticles by the exploitation of MN-mediated delivery, the field of $\mathrm{AD}$ research is not dissimilar to that of other neurological agents, such as antipsychotic drugs (Section 5.5.1), in that this platform has remained relatively unexplored in the potential delivery of agents for the treatment of $\mathrm{AD}$. While MN-mediated delivery for the specific treatment of $\mathrm{AD}$ has yet to be explored in combination with nanomedicine technology, two independent studies have successfully demonstrated delivery of the anticholinesterase inhibitor, donepezil, with different $\mathrm{MN}$-assisted approaches. Donepezil has a very long half-life and therefore lends itself readily as a candidate to achieve a sustained therapeutic release [277]. Inspired by the success of rivastigmine patches, Kearney et al. employed hydrogel-forming arrays with PMVE/MA reservoirs loaded with donepezil. Evaluation of the performance in vivo in rat models demonstrated successful delivery by this platform, producing detectable plasma levels of $51.8 \pm 17.6 \mathrm{ng} / \mathrm{mL}$ at $24 \mathrm{~h}$ [278]. In a different MN-based approach, Kim et al. encapsulated a high donepezil hydrochloride content (up to $78 \%$ w/w) exclusively within dissolving MN tips without any severe loss of mechanical strength, and $95 \%$ of tip content was delivered through the porcine skin in 5 min. Besides, evaluation of pharmacokinetics in rats was cited as satisfactory as evidenced by the $\mathrm{C}_{\max }$ exhibited by $\mathrm{MN}$ delivery 4 times higher than that of oral administration at $9 \mathrm{ng} / \mathrm{ml}$. Furthermore, a total of $69 \%$ of donepezil entrapped within the MN tip was detected in plasma at $24 \mathrm{~h} \mathrm{[279].} \mathrm{While} \mathrm{the} \mathrm{results} \mathrm{of} \mathrm{both} \mathrm{of} \mathrm{these} \mathrm{studies} \mathrm{are} \mathrm{cautiously}$ indicative of a sustained release, sampling did not extend beyond $24 \mathrm{~h}$ to confirm this assumption in either instance, however, successful delivery of a potential treatment of AD was demonstrated in both cases. The benefit of transdermal products in the treatment of AD is well documented, and these studies offer substantial promise in support of future MN mediated delivery for AD therapy. However, further work is now required in evaluating this platform in conjunction with nanocarriers to fully realise this potential of the dual-delivery approach to attain a minimally invasive sustained-release alternative to currently available oral or transdermal therapy.

\subsection{Diabetics/Insulin}

Insulin is a compound delivered via subcutaneous injection to treat type 1 diabetes [283], in those patients who are specifically unable to produce enough insulin [284,285]. Not only is this route of delivery considered by many to be uncomfortable and painful, but it is associated with an increased risk of infection, and importantly, it generates a high amount of sharps waste 
[283]. One of the most promising types of MN-assisted insulin delivery systems is the glucoseresponsive MNs [286], explored in section 4.2.5 "Stimuli-responsive smart MN arrays" [286]. In a similar approach, Chen et al. [287] developed smart MN based on SF combined with semiinterpenetrating network hydrogel for glucose-responsive insulin delivery, aiming to replace the subcutaneous self-injection. The authors achieved sustained and stimuli-responsive insulin delivery, controlling the polymer degradation into the skin. The glucose-responsive system consisted of a two-layer patch, fabricated with silk fibroin combined and phenylboronic acid/acrylamide hydrogel (needles layer) and the base layer prepared only with silk fibroin. A porous system developed by submitting the MN to the lyophilisation improved the stimuliresponsive system, able to release insulin only when the hyperglycaemia was detected, based on the reaction of glucose binding reversibly and boronate ester in aqueous solution, releasing the insulin when the glucose is detected. The SF was found to act as a suitable polymeric matrix former capable of delivering sufficient amounts of insulin ID. Wang et al. [288] also describe MNs insulin-loaded formed with SF as a polymeric matrix. This material is biocompatible, non-toxic, degradable, and presented slow insulin release, indicating the MNs release was maintained up to $60 \mathrm{~h}$, providing the future promise of the platform for the application of longacting insulin delivery.

As an alternative to subcutaneous insulin delivery, dissolving composite MNs containing insulin-loaded $\mathrm{CaCO}_{3}$ microparticles were developed by Liu et al. [289]. These $\mathrm{CaCO}_{3}$ MPs containing insulin were obtained by precipitation in the presence of PVP. The new MNs $(5,10$

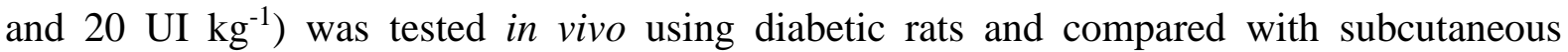
administration (5 $\left.\mathrm{UI} \mathrm{kg}^{-1}\right)$. Therapeutic plasma insulin levels were found to be maintained for a longer period when insulin was delivered via MNs compared with SC injection of a similar dose [289]. This may be explained by the increased time required for the systemic absorption of insulin delivered via MNs. The extension of release rate achieved with MNs insulin microparticles loaded was beneficial to reduce the risk of hypoglycaemia [290].

Prolonged-release kinetics utilising MNs for insulin delivery has also been demonstrated by Kim et al., in which they developed powder-carrying insulin MNs. The method of delivery was based upon creation of micro-cavities from MNs application to the skin and, subsequently, introducing the active agent on powder form on it using a micropillar implantation system to compact the insulin homogenously into the microcavities. The in vivo studies were able to prove the concept of the new MNs, achieving a prolonged release when compared with subcutaneous injection. This new approach can be applied to a range of powder drugs, with 
further beneficial impacts upon minimising the side effects and improving the long-term stability, one of the issues related to insulin [291]. Seong et al. achieved the prolonged release of insulin using double-layer MNs with swellable tips composed by polystyrene-blockpoly(acrylic acid) with a bullet-shaped array, and non-swellable polystyrene baseplate. To load the insulin only in the tips, a mild drop/dry procedure was applied, achieving $60 \%$ of release after $12 \mathrm{~h}$ in vitro studies. The in vivo studies showed a prolonged release from the swellable MNs resulting in a gradual decrease in blood glucose levels, explained due to the ability of the MN to uptake the body fluid in the tips, allowing the insulin release via diffusion [292]. Chen et al. developed a basal-bolus insulin regimen integrated $\mathrm{MN}$ patch for intraday postprandial glucose control, with multiple kinetics, which provided physiologic insulin coverage when tested in diabetic rats. The new technology comprised an array containing the short, intermediate and long-acting insulin, separated by three separated projections 9x9 for each type of insulin, totalling a patch with $81 \mathrm{MN}$ projections. To develop the integrated MN patch, formulations containing the three different insulin were prepared separately. To deliver the immediate insulin, a hydrogel with gelatin was prepared. For intermediate insulin, a crosslinked blend of hydrogel composed with gelatin and genipin was developed. Moreover, to deliver the long-acting insulin, a cross-linked hydrogel containing hyaluronic acid and 1,4butanediol diglycidyl ether was used. The in vivo studies showed sustained insulin delivery, because of the simultaneous bolus provided under the $\mathrm{MN}$ application, reducing the excessive intraday glucose fluctuations that occur in response to meal ingestion, showing the potential of this new MN system as a substitute for multiple daily injections [293].

\subsection{Cancer}

Traditional cancer treatment includes surgery, chemotherapy and, radiation. However, the effects of these procedures may damage not only the tumour tissue but also normal bodily tissues [294]. The exploitation of the properties of nanocarriers may overcome such limitations of conventional treatment, resulting from their nanometre scale, high surface-to-volume ratios, favourable drug release profiles, and targeting modifications, which may, in turn, may permit drug release in a sustained manner and subsequently increased drug localisation and cellular uptake within the target tumour tissue [295]. Current nanocarrier based drug delivery systems for cancer treatment, which are already under research and development, include liposomes, polymeric micelles, dendrimers, nanospheres, nanocapsules, and nanotubes [294]. Nanoformulations that have already been marketed include Doxil ${ }^{\circledR}$ (liposomal doxorubicin) [296], Abraxane ${ }^{\circledR}$ (nanoparticle-based albumin-bound paclitaxel) [297] and Onivyde ${ }^{\circledR}$ 
(liposomal irinotecan) [298]. However, their administration, by necessity, is typically invasive (IV infusion) and may lack direct access to more restricted tumour sites, thus resulting in poor accumulation or non-localised distribution. Consequently, a minimally invasive cancer treatment associated with MN mediated delivery always provides broad appeal as a result of ease of use, non-invasive controllable administration and predominantly a potential synergistic effect [55]. Besides, some anti-cancer $\mathrm{MN}$ nanocarrier based systems have already demonstrated particular promise for the treatment of certain cancers. Cisplatin, a first-line chemotherapeutic agent, has recently been formulated within tumour-targeting $\mathrm{pH}$-responsive lipid nanoparticles by Lan et al., with the resultant nanoparticles embedded in dissolving MNs as a means to mediate the safe and efficient transdermal delivery for cancer therapy. Nanoparticles exhibited a high drug loading weight (80\%), and substantially increased the solubility of cisplatin and enhanced its antitumour efficiency. Additionally, a sustained release over a period of $72 \mathrm{~h}$ in dialysis membranes in vitro was demonstrated. In in vivo studies utilising a xenograft tumour $\mathrm{BALB} / \mathrm{c}$ murine model, $\mathrm{MN}$-mediated delivery arrays significantly increased cytotoxicity and apoptosis in cancer cells with an apoptotic index of $58.6 \%$, resulting in significantly reduced tumour volume and weight. Moreover, it was established that serum platinum, pulmonary toxicity, hepatotoxicity, and nephrotoxicity were not detected in vivo, indicating that the technique was bio-safe [126]. recently delivered DOX encapsulated PLGA nanoparticles to the oral cavity in the treatment of carcinoma. Stainless steel MNs arrays of $700 \mu \mathrm{m}$ tip height were coated with nanoparticles of average diameters of $137 \mathrm{~nm}$ and applied to porcine cadaver buccal tissue. Evaluation of MN treated tissues utilising confocal microscopy proved that DOX could diffuse both vertically and laterally into the tissues and produced cellular cytotoxicity, with nanoparticles detected at a penetration depth greater than $1 \mathrm{~mm}$ from the point of insertion from the MN tip. In contrast, administration of DOX by hypodermic injection into porcine buccal tissue reinforced significant leakage of the injected volume (about $25 \%$ of the injected $80 \mu \mathrm{l}$ ). Moreover, nanoparticles demonstrated a substantial prolonged release, as only $34 \%$ of the encapsulated DOX was released in vitro over a $24 \mathrm{~h}$ period. This sustained release of DOX, especially the release of the remaining $66 \%$ of the drug still contained within the nanoparticles is a beneficial trait for chemotherapy because constant exposure of tumour cells to DOX is expected to result in high cytotoxicity [299]. MNmediated delivery has also extended to some anti-inflammatory agents which possess residual adjunctive cytotoxic properties, such as that of ketoprofen, as demonstrated by Vučen et al. Ketoprofen nanocarriers composed of PLA were found to exhibit a sustained release over 7 days under the sink conditions using Slide-A-Lyzer dialysis cassettes. They demonstrated an 
enhanced release over $24 \mathrm{~h}$ in porcine tissue following application to excised skin pre-treated with silicon MNs [300].

As well as chemotherapeutic agents, nanoformulated monoclonal antibodies have also been utilised for enhanced cancer immunotherapy by MN-mediated delivery. Self-degradable MN patches have been investigated by Wang et al. for the potential treatment of melanoma, through sustained delivery of anti-PD-1 (aPD1) in a physiologically controlled manner. MN-patches composed of biocompatible HA were incorporated with glucose oxidase (GOx) loaded $\mathrm{pH}$ sensitive dextran nanoparticles encapsulated with aPD1. The generation of acidic environment promoted the self-dissociation of nanoparticles and subsequently resulted in the substantial release of aPD1. In this study, robust immune responses in a B16F10 mouse melanoma model were produced from a single aPD1-GOx MN patch application, in comparison to MN lacking a degradation trigger or that of intratumoral injection of free aPD1 at the same dose. Moreover, it was hypothesised that as a result of the superior immune response, that this administration strategy could be integrated with other immunomodulators (such as anti-CTLA-4) to achieve combination therapy for enhancing antitumour efficacy [301]. The studies described here demonstrate the sustained delivery of various therapeutic nanoformulation outlets involved in potential anti-cancer therapy, thus demonstrating early promise for the future of MN-mediated delivery in the treatment of cancer, and supports the further investigation of this non-invasive delivery platform.

\subsection{Skin diseases}

The skin is the principal protective barrier of the human body. However, it is susceptible to various diseases and disorders such as psoriasis, viral warts, cold sore, fungal infections, soft tissues infections and leishmaniasis. Most of these diseases are chronic, difficult to treat and, thus, seriously affecting peoples quality of life [302]. While in mild cases, topical therapy is mostly recommended, systemic therapy (oral or parenteral) for is widely indicated [303]. Unfortunately, topical therapy efficacy reduced by the skin barrier properties. For example, it has been demonstrated that when a topical cream spreads on the skin surface, only $10-20 \%$ or less of total drug loaded in cream could permeate through the skin [304]. Also, the efficacy of many of the systemic therapies is suboptimal this because drug administration through the gastrointestinal tract or circulatory system sometimes increases drug consumption or reduces local drug concentration per unit area of skin. This is mostly associated with numerous side effects and toxicity especially for drugs such as cyclosporin A (CyA), methotrexate (MTX) 
and the antifungal agent- itraconazole [114,303,304]. Owing to limitations of the existing methods of treatment, researchers are committed to performing innovative investigations into drug delivery methods employing MNs technology alone or, in combination with long-acting formulation for efficient intradermal delivery of many of the therapeutic agents which are used to treat dermatological conditions [16,303-305].

Here, we focused on one of the most common skin diseases, namely, psoriasis as a case study, where we could track the up-to-date advancement made in using MNs-assisted delivery of long-acting formulations approach for localised and sustained intradermal drug delivery and minimising drug systemic exposure. Psoriasis is a chronic inflammatory autoimmune skin disease that affects $1-3 \%$ of the population [306,307]. Currently, several treatment strategies are in use, including photochemotherapy with UVA (PUVA), retinoids, CyA, MTX and recently biologics $[308,309]$. The latter three medications are widely used to treat the moderate to severe cases of psoriasis and, administered systemically (orally or parenterally), which are associated with many side effects and toxicity. Over the past two decades, many researchers have investigated utilising MNs alone or in combination with long-acting formulations for efficient intradermal delivery of the above-mentioned therapeutic agents to improve their efficacy and safety. Recently, Tekko et al. achieved the sustained release of MTX over $24 \mathrm{~h}$ with even reduced drug peak concentrations using a newly developed PVA/PVP-based hydrogel-forming MNs formulation [63]. The innovative approach achieved Cmax (35.1 nM) of MTX in $24 \mathrm{~h}$ post-MNs application against $\mathrm{C}_{\max }(57.4 \mathrm{nM})$ in $1 \mathrm{~h}$ for orally administered MTX aqueous solution in rats. Considering that some of the side effects (i.e. nausea and vomiting) of MTX are mostly related to its high spike blood concentrations [310], this novel MNs-mediated delivery approach has the potential to reduce/avoid these side effects, which are associated with systemic delivery. This drug delivery system was originally developed to deliver MTX to treat juvenile idiopathic arthritis; however, also it may be tailored to treat psoriasis, in which MTX is also licenced for use. In another study, Tekko et al. combined NCs and MNs technologies to localise and sustain release of MTX as a potential treatment for psoriasis to minimise the systemic exposure in order to avoid the drug systemic toxicity and side effects [15]. The authors prepared poorly water-soluble MTX-NCs from its freely watersoluble salt MTX Na by bottom-up method, namely acid-base neutralisation accompanied with sonification. The MTX NCs (average diameter of $700 \mathrm{~nm}$ ) were then incorporated into the projections of dissolving MNs with a drug loading of $2.48 \mathrm{mg} / \mathrm{array}$. The MNs projections dissolved within 20 minutes upon insertion and deposited around $25 \%$ of their drug payload in 
the skin (in vitro). The in vivo study in Sprague Dawley rats revealed that the MTX NCs-loaded MNs were able to deposit approximately $25 \%$ of the loaded MTX NCs in the skin, which acted, in turn, as a drug depot and released the drug in a sustained manner over $72 \mathrm{~h}$ while minimising MTX systemic exposure in comparison with orally administered MTX Na (the control). For example, after $24 \mathrm{~h}$ from MN application, approximately $312.70 \mu \mathrm{g} / \mathrm{g}$ of MTX was retained in the skin at the application site. This was 322-fold higher than the amount of MTX $(0.942 \mu \mathrm{g} / \mathrm{g})$ retained in the skin after oral administration of MTX Na. even after $72 \mathrm{~h}$ from MNs application, around $12.5 \%$ of the deposited drug was still retained at the application site. This approach was not only effective in delivering and retaining the drug for a prolonged period but also reduced the systemic exposure remarkably. Although the delivered MTX dose from MNs was approximately 25-fold higher than that delivered by oral administration, the maximal blood concentration of MTX was only $40 \%$ of that measured after oral administration of MTX Na.

Intradermal delivery of CyA using dissolving MNs has also been investigated. Benefiting from its intrinsic physicochemical properties, large molecular size (1 $202.61 \mathrm{~g} / \mathrm{mol})$ and, hydrophobic properties ( $\log \mathrm{P}=2.92$ ), Jeong et al. developed hydroxypropyl cellulose-based dissolving MNs loaded with CyA for localised and sustained intradermal delivered [311]. The authors indicated that these dissolving MNs performed well in vivo using a rat model, with a longer period of therapeutic effect locally compared with oral administration. This was demonstrated by CyA administered with MNs maintained a CyA concentration in plasma above $5 \mathrm{ng} / \mathrm{ml}$ for $72 \mathrm{~h}$ compared to $24 \mathrm{~h}$ for CyA administered orally. The studies described here demonstrate that MNs-assisted delivery of long-acting formulations offers an outstanding opportunity to enhance therapeutic efficacy (by delivering the drug directly to the affected site and maintain the effective drug concentration for prolonged periods) and safety (by minimising their systemic exposure). Therefore, these studies support further investigation using this approach to deliver other therapeutic agents to not only treat psoriasis, but also other skin diseases and disorders.

\subsection{Ocular diseases}

MNs have been widely explored for the delivery of the drugs to an ocular tissue, to both the anterior and posterior segments of the eye [312]. The cornea and sclera are the outermost layers of the eye which provide the greatest challenge for drug diffusion to the inner ocular tissue, thus limiting the bioavailability of the drug [313]. As depicted in Figure 10, MNs find a 
potential application in ocular delivery due to their ability to decrease the resistance to the diffusion physically. Hollow MNs, have received the greatest research interest in this aspect, as well as solid MNs, which have been extensively researched for periocular drug delivery. Other designs, including hydrogel-forming MN, as well as interlocking MNs, have also been studied for their ability to enhance ocular drug penetration [49].

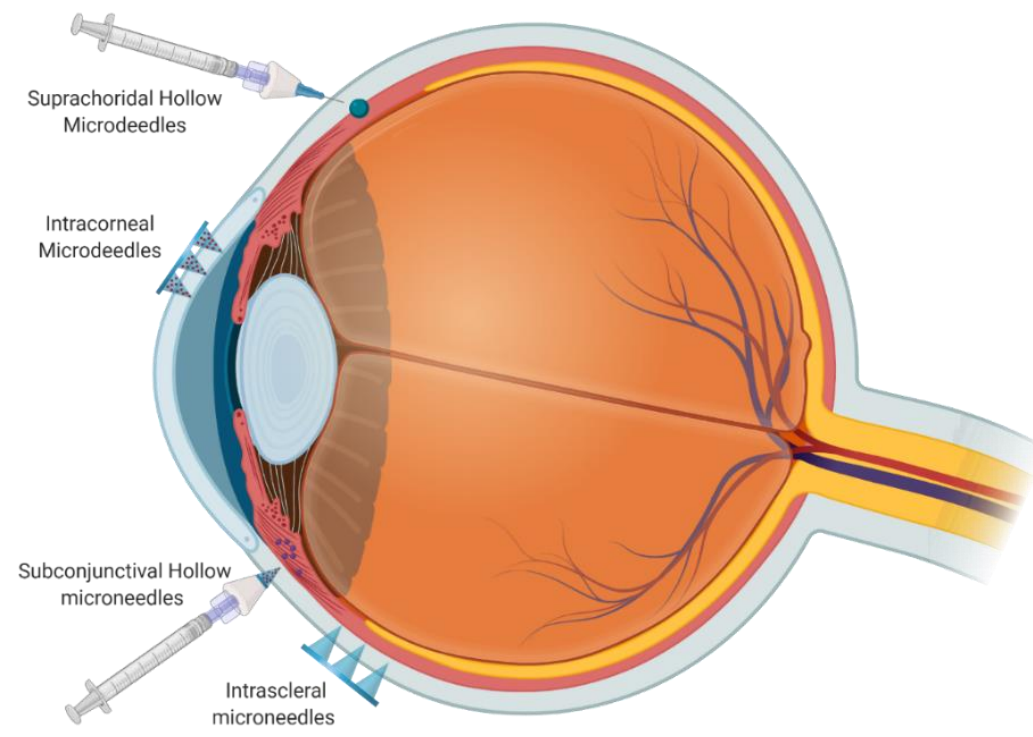

Yiu et al. reported the assessment of suprachoroidal delivery of adeno associated viruses (AAV) using a transscleral Hollow MN system in non-human primates using, comprising of 30 gauge $700 \mu \mathrm{m}$ MNs. The suprachoroidal delivery successfully leads to transient GFP expression on the retinal layer for up to 3 months following $\mathrm{MN}-$ mediated deliver in rhesus macaques. The study suggested that transscleral MN systems could be successfully used for efficient gene delivery for the management of retinal disorders [314].

MN could also be used for the preparation of intrascleral implants. Thakur et al. reported the preparation of intrascleral implant using poloxamer gel loaded with the fluorescein sodium dye as a model drug. This hydrogel was injected by using the hollow MN device, which was fabricated using the hypodermic needles of different lengths $(400-600 \mu \mathrm{m})$. This study suggested optimum concentration of hydrogels could be used as an efficient tool for preparation of intrascleral implant that could balance the drug loading as well as the force of injection and sustained release [315]. Rowe-Rendleman et al. have used intrascleral injections of medroxyprogesterone, triamcinolone and, fluocinolone for studying the effect of steroid in the prevention of retinal neovascularisation caused by intraocular injection of lipid hydroperoxide 
(LHP). The intrascleral injections were made with a micro-surgical approach by piercing the epi-sclera and depositing drug directly in the sclera for diffusion towards the choroid using 100 $\mu 1$ syringe and 30 gauge needle as reported by Ueda et al. The study suggested that the intrascleral injections were well tolerated in the rabbit eye and did not lead to increase in the intraocular pressure, as well as did not posed any damage to retinal architecture [316].

One of the main challenges for drug delivery to the posterior segment is the limited delivery coverage leading to less profound therapeutic effect. Hwan Jung et al. reported the suprachoroidal delivery of collagenase enzyme along with latex microparticles leading to enhanced ocular coverage for distribution of microparticles. Hollow MN with a length of 750 $\mu \mathrm{m}$ and 30 gauge was used for the for delivery of $50 \mu \mathrm{l}$ liquid containing microparticles and collagenase enzyme blend [317]. The same group also reported the delivery of hydrogel using a similar hollow MN system. HA-based (1\%) microparticles along HA-based (4\%) hydrogel loaded with model drug particles were delivered using hollow MNs. This strategy could be used for enhancing the sustained drug delivery effect of various transscleral and suprachoroidal drug delivery systems [318]. Jiang et al. also reported intrascleral drug delivery utilising hollow MNs for the delivery of various formulations. An in-house $\mathrm{MN}$ device containing a hollow $\mathrm{MN}$ and drug reservoir connected by a rubber tubing was developed. The hollow MN had an elliptical tip opening with a long axis of $\sim 100 \mu \mathrm{m}$, and a short axis of $\sim 40 \mu \mathrm{m}$ and, the length of MN was controlled from the injector. Furthermore, this system was used for the infusion of sulforhodamine as well as dye loaded nanospheres and microparticles into the human cadaver sclera, in which MN of 700-1080 $\mu \mathrm{m}$ length was used. The study suggested that a higher quantity of liquid could be infused in the medial sclera when compared to the anterior and posterior scleral tissue. Additionally, the study suggested that the use of such MN device could have a potential therapeutic application for delivery of small volumes of the drug as well as nanoparticles by the intrascleral route [224].

Kim et al. reported a miniaturised porous silica-based MN array backed on PVA membrane for management of ocular melanoma. The MNs were fabricated by standard photolithographic patterning of silica wafers followed by the formation of the backing membrane using PVA lamination. Finally, DOX was loaded on the surface of MN. The MN-assisted administration was found to sustain the release of DOX for up to $24 \mathrm{~h}$ by the application of chemical bonding. Maher et al. suggested the application of a self-adhesive and interlocking MN system for sustained ocular drug delivery. The MN consisting of dimensions of $1500 \mu \mathrm{m}$ length and 275 $\mu \mathrm{m}$ base diameter, were fabricated using PVA. The interlocking mechanism leads to the 
increase in the adhesion force, which can be further translated to sustained release of the drug. Although detailed in vivo studies are required for validation of interlocking MN systems. this could be used as a potential tool for sustained intrascleral drug delivery [319]. Saraswathy et al. reported the preparation of particle loaded hydrogel ladened with HA-based MNs for enhanced ocular drug delivery. The moxifloxacin loaded HA MNs were prepared and sustained in the collagen-based cyrogel; the cyrogel was further crosslinked with 4-armed PEG succinate glutarate. The system was found to sustain the release of moxifloxacin for up to 7 days in invitro evaluation. Further in-vivo studies are required for the assessment of the biocompatibility of this system [320]. MN patches were used for delivery of amphotericin B (AmB) by Roy et al. The MN patches were prepared using a PVA and PVP mixture using PDMS template, and the dimension of MN was $552 \mu \mathrm{m}$ length and with $1.64 \mu \mathrm{m}$ diameter. Liposomal formulation of $\mathrm{AmB}$ was delivered following the application of solid $\mathrm{MN}$ to form pores on the corneal surface, which was found to lead to higher retention of AmB in the cornea. The MN-assisted drug delivery system also demonstrated complete clearance of the Candida albicans infection under $48 \mathrm{~h}$ in in-vitro conditions [321]. Thakur et al. have further reported a rapidly dissolving MN-based drug delivery system for the intrascleral drug delivery. The fluorescein sodium and FITC dextran $(70 \mathrm{kDa}$ and $150 \mathrm{kDa})$, were selected as model molecules. The MNs were prepared from drug-loaded PVP hydrogels and characterised for mechanical properties, and ex-vivo tissue penetration and distribution. It was observed that rapidly dissolving $\mathrm{MN}$ were able to dissolve up to $87 \%$ of the mass in less than three minutes. The biodistribution studies suggested that after MN dissolution, an intrascleral depot formed of FITC-dextran and, lead to an increase of more than $60 \%$ drug permeation across the scleral tissue. Thus, suggesting that it could be a potential intrascleral drug delivery system which could be used for minimally invasive drug delivery of macromolecules [312].

Than et al. studied the fabrication and evaluation of double-layered $\mathrm{MN}$ systems for transcorneal drug delivery. The MN patches were prepared using HA and methylated HA (MeHA), where the outer layers were made using the hydrophobic MeHA, and the inner core consisted of HA. The MN was able to sustain the drug release for up to $120 \mathrm{~h}$. The drug delivery system was also found to be biocompatible, as it does not lead to any anatomical changes post insertion for up to 7 days as well as no change in corneal opacity. The MN system was used to deliver the DC101 by the intracorneal route. The in-vitro and in-vivo assay suggested that the MNs lead to a marginal reduction of up to $90 \%$ in the neo-angiogenesis when compared with topical and intraperitoneal application of the same antibody along with less macrophage 
accumulation. The authors also achieved two weeks of sustained-release when HA-IgG conjugates are loaded in MeHA matrix. Based on this proof of concept, choosing or mixing with PLGA polymer matrix or conjugates, drug release kinetics from several days to months can be tailored [49]. Based on these sustained and long-acting MN approaches, various molecules can be readily delivered by localized, controlled manner that provides a pain-free, cost-effective and home-based solution for many ocular diseases.

\section{Lab to market considerations and challenges}

The primary purpose of the MN-mediated delivery systems is to reduce the burden of daily pills fatigue or painful injectable drug administration and, thus, improve patient compliance. Sustained-release drug delivery assisted by MNs can simplify drug administration, accomplish therapeutic concentrations for a specified period and, evade potential systemic toxicity. Although such an approach has many advantages in comparison with the conventional drug delivery systems, it has some limitations, which should be considered with a system designed to deliver such drugs. Firstly, unlike the conventional hypodermic needles, MNs are micronsized devices and the amount of drug, which can be delivered, is limited. Secondly, to minimise drug wastage and increase delivery efficiency, bilayer MNs, where the drug is loaded in the MNs projections only, should be considered. Thirdly, to maximise drug loading per unit area in the MNs, poorly water-soluble forms or its nanocrystals should be the first option, whenever is possible, as using controlled release polymers such as PLGA and PLA, could reduce the amount of MNs drug loading capacity dramatically. Moreover, to avoid accumulation in the body, biocompatible/biodegradable polymers with molecular weight $<60000$ Da should be used to form the matrix of MN tips. Further special attention should be paid to MNs design and geometry which allow high drug loading, sufficient mechanical strength and, maximum insertion capability, such as, pedestal MNs or long MNs with intermediate interspacing (e.g. around $100 \mu \mathrm{m}$ ), but their length should not exceed $1 \mathrm{~mm}$ to avoid stimulating the nerve endings in the dermis.

Finally, the use of controlled-release technology is also reliant on the disease state or therapeutic effect desired. To transfer MNs products from the bench to the bedside, several challenges need to be addressed. Although the development of MNs assisted drug therapy has undergone substantial progress and contributed significantly toward trans-intradermal delivery, numerous limitations persist for large-scale manufacturing, regulatory requirements, and patient acceptance. As MN delivery systems are a comparatively new technology in the case 
of commercialisation, their translational capability could be affected by complex formulation difficulties. Therefore, the creation of good manufacturing practice (GMP) facilities that set out and establish specific guidelines for $\mathrm{MN}$ formulation manufacture is necessary [322].

Table 2. Considerations for MN product development [322-324].

\begin{tabular}{|c|c|c|}
\hline Pharmaceutical Considerations & Preclinical Considerations & $\begin{array}{l}\text { Clinical evaluation for the } \\
\text { commercialisation }\end{array}$ \\
\hline $\begin{array}{l}\text { Reduce complexity in the } \mathrm{MN} \\
\text { fabrication }\end{array}$ & $\begin{array}{l}\text { Evaluation of } \mathrm{MN} \text { application in an } \\
\text { appropriate animal model }\end{array}$ & $\begin{array}{l}\text { Evaluation of therapeutic efficacy, } \\
\text { safety/toxicity (acute and chronic) } \\
\text { in humans }\end{array}$ \\
\hline $\begin{array}{l}\text { Development of global regulatory } \\
\text { or pharmacopeial standards for } \\
\text { MN testing }\end{array}$ & $\begin{array}{l}\text { Optimisation of } \mathrm{MN} \text { insertion } \\
\text { efficiency and application } \\
\text { reproducibility in pre-clinical and } \\
\text { clinical skin models /subjects }\end{array}$ & $\begin{array}{l}\text { The requirement of clear } \\
\text { regulatory guidelines }\end{array}$ \\
\hline $\begin{array}{l}\text { Optimisation of } \mathrm{MN} \text { application } \\
\text { with or without applicator device } \\
\text { for reproducible insertion }\end{array}$ & $\begin{array}{l}\text { An adequate understanding of in- } \\
\text { vivo behaviour of } \mathrm{MN} \text { insertion } \\
\text { and drug deposition as well as drug } \\
\text { absorption through the intradermal } \\
\text { space }\end{array}$ & $\begin{array}{l}\text { Understanding of the biological } \\
\text { interaction of } \mathrm{MN} \text { with the } \\
\text { biological environment in the body } \\
\text { of patients }\end{array}$ \\
\hline $\begin{array}{l}\text { Pharmaceutical stability } \\
\text { (Chemical and Physical) }\end{array}$ & $\begin{array}{l}\text { PK, metabolism, disease } \\
\text { outcomes, and potential toxicities } \\
\text { in small animals }\end{array}$ & $\begin{array}{l}\text { Simplification of development } \\
\text { pathways from invention to } \\
\text { commercialisation to minimise } \\
\text { time and expense }\end{array}$ \\
\hline $\begin{array}{l}\text { Whether a sterile or low bioburden } \\
\text { device will be acceptable to } \\
\text { regulators }\end{array}$ & $\begin{array}{l}\text { confirmatory of drug PK and PD } \\
\text { assessments in large animals } \\
\text { include rhesus macaques and pig }\end{array}$ & $\begin{array}{l}\text { Investigational new drug (IND) } \\
\text { route }\end{array}$ \\
\hline $\begin{array}{l}\text { MN array patch design } \\
\text { optimisation for the human use }\end{array}$ & $\begin{array}{l}\text { Identifying the right animal model } \\
\text { for the animal to human translation }\end{array}$ & \\
\hline $\begin{array}{l}\text { Scaled-up manufacture of } \mathrm{MN} \\
\text { patches with sizes suitable for } \\
\text { delivery of high drug doses }\end{array}$ & & \\
\hline
\end{tabular}

Successful MNs system development requires emphasis on reliable and repeatable application, a comprehensive survey of the user experience and, minute details of the MN fabrication process. MN enabled long-acting delivery, both hollow and solid MN, pose specific challenges. Answers to all these limits typically necessitate complex solution based on multidisciplinary approached with an in-depth insight of biology, skin physiology, physics, chemistry and bioengineering [325]. Mainly, converting nanomedicine facilitated long-acting MN formulations into the clinical product, there are further inherent challenges based on structural and chemical complexity of nanomedicines [324,326] Any added complexities to this MN technology would require demonstrating significantly superior benefits to the patients, due to the further costs and complexity in the manufacturing side. MN drug product-centric factors such as sterility of the MN, potential immunological effects, assurance of MN insertion, 
deposition of $\mathrm{MN}$ material into the skin, the potential for $\mathrm{MN}$ re-use, uniformity of content and packaging, should be considered before technology transfer from lab to large scale product development for clinical studies [323]. Consideration to each one of these Research \& Development criteria must be part of any systematic evaluation of GMP manufacturing to guarantee proper development and effective clinical translation. Multi-step MN production at GMP scale-up, continuous, and automatic on-line control with minimal human interference will be helpful to ensure product consistency, reproducibility and, quality. At present, most long-acting strategies are centred on improving the pharmaceutic and pharmacokinetic (PK) aspects in preclinical models. Small-animal models such as mice and rats provide screening of $\mathrm{MN}$ application, PK and, pharmacodynamics (PD) assessments. In these systems, improving product delivery, drug tissue targeting, metabolism, disease outcomes and, potential toxicities can be checked. Before proceeding to human clinical trials, evaluation in larger animal models, including rhesus macaques and pigs are now necessary for further in-depth and detailed confirmation of drug PK and PD assessments [327,328].

Those involved in charting the future evolution of $\mathrm{MN}$ assisted long-acting therapeutics should focus on not only the production efficacious delivery system but also patient-centred one. Although the development of nanotherapeutics has the potential to address the limitations of current MN long-acting technology. Largely, these hurdles can be categorised with respect to pharmaceutics (formulation, scalability, usability), PK (absorption, disposition, safety) and pharmacodynamics (efficacy against disease), and clinical evaluation as described in Table 2. However, their actual utility in the clinic and acceptance by patients are being determined in broader populations. Various approved medical and cosmetic products using MNs that are sold around the world with low cost and good quality boost the confidence to explore the MN technology further. Based on industrial interest and possible benefits for patients with MN device technology, recently, the US FDA published draft guidance for quality control criteria for MN manufacture and acceptance for cosmetic applications [329], would be extended to therapeutics $\mathrm{MN}$ once some clinical trials progress with positive outcomes.

\section{Conclusion/Future prospects}

Recently, nanotechnology-based transdermal drug delivery platforms have gained renewed interest. Particularly, in combination with $\mathrm{MN}$ to ascertain a way to deliver a wide range of nanotherapeutics intradermally and, sustain the release of the therapeutic levels with only a single MN application. Urgently overcoming this unmet patient need is now necessary to 
address both clinical needs and patient adherence. $\mathrm{MN}$ technology is in top first list amongst “10 Emerging Technologies 2020” by World Economic Form [330]. Overall, MN technology is still in its early stage of clinical translation. First MN product with zolmitriptan (Qtrypta $\left.{ }^{\mathrm{TM}}\right)$ expected to be launch in the coming year that developed by Zosano Pharma Corp. This MN product NDA completed the first full review from US FDA and now the company have been trying to address the FDA concerns related to inconsistencies on drug plasma levels during the clinical trial from different manufacturing batches [331]. Therefore, simple, scalable, and userfriendly MN drug delivery platforms should be established. Once such a novel MN-assisted treatment is launched, it could be used to address the most challenging life-threatening and chronic diseases, such as HIV/AIDS, cancer, diabetes, cardiovascular disorders etc. At present, most long-acting MN strategies are focused on optimising PK and PD aspects in preclinical models [118,167,266]. Although MNs presents are able to deliver both hydrophobic and hydrophilic, small and large molecules for long-acting release, more studies need to be completed at the preclinical stage to support this. Additionally, efficient collaboration between academia, industry, non-profit organisations and, hospitals is now necessary to bridge the gap between the laboratory and meeting the patient. PATH, an international, non-profit, global health organization, has established the "Microarray Patches-Centre of Excellence" to bring all stakeholders under one umbrella to hasten MN manufacturing scale-up and, also the regulatory and clinical trial processes [332]. Finally, health and regulatory authorities should establish and enforce definitions ( $\mathrm{MN}$ as a pharmaceutical product or device), quality test standards, guidelines and, regulatory issues for the $\mathrm{MN}$ drug delivery platform [325]. More investigational new drug (IND) type studies focusing on in vivo efficacy and long-term toxicity will need to be done. With more preclinical studies (and potential clinical studies) expected to take place over the next few years, we believe that more encouraging data will be reported to demonstrate the versatility of $\mathrm{MN}$ as a leading platform of choice for long-acting drug delivery. This could be the major step that is needed for the field to make the necessary progress towards fully realising the full potential of this novel drug delivery platform.

\section{References}

[1] A.Z. Alkilani, M.T.C. McCrudden, R.F. Donnelly, Transdermal drug delivery: Innovative pharmaceutical developments based on disruption of the barrier properties of the stratum corneum, Pharmaceutics. 7 (2015) 438-470. https://doi.org/10.3390/pharmaceutics7040438.

[2] S. Sharma, K. Hatware, P. Bhadane, S. Sindhikar, D.K. Mishra, Recent advances in microneedle composites for biomedical applications: Advanced drug delivery technologies, Mater. Sci. Eng. C. 103 (2019) 109717. https://doi.org/10.1016/j.msec.2019.05.002.

[3] R.F. Donnelly, R. Majithiya, T.R.R. Singh, D.I.J. Morrow, M.J. Garland, Y.K. Demir, K. Migalska, E. Ryan, D. Gillen, C.J. Scott, A.D. Woolfson, Design, optimization and 
characterisation of polymeric microneedle arrays prepared by a novel laser-based micromoulding technique, Pharm. Res. 28 (2011) 41-57. https://doi.org/10.1007/s11095-0100169-8.

[4] A.J. Harvey, S.A. Kaestner, D.E. Sutter, N.G. Harvey, J.A. Mikszta, R.J. Pettis, MicroneedleBased Intradermal Delivery Enables Rapid Lymphatic Uptake and Distribution of Protein Drugs, Pharm. Res. 28 (2011) 107-116. https://doi.org/10.1007/s11095-010-0123-9.

[5] A.D. Permana, M.T.C. McCrudden, R.F. Donnelly, Enhanced Intradermal Delivery of Nanosuspensions of Antifilariasis Drugs Using Dissolving Microneedles: A Proof of Concept Study, Pharmaceutics. 11 (2019) 346. https://doi.org/10.3390/pharmaceutics1 1070346.

[6] H.L. Quinn, M.-C. Kearney, A.J. Courtenay, M.T.C. McCrudden, R.F. Donnelly, The role of microneedles for drug and vaccine delivery, Expert Opin. Drug Deliv. 11 (2014) 1769-1780. https://doi.org/10.1517/17425247.2014.938635.

[7] Y.A. Gomaa, M.J. Garland, F. Mcinnes, L.K. El-khordagui, C. Wilson, R.F. Donnelly, European Journal of Pharmaceutics and Biopharmaceutics Laser-engineered dissolving microneedles for active transdermal delivery of nadroparin calcium, Eur. J. Pharm. Biopharm. 82 (2012) 299307. https://doi.org/10.1016/j.ejpb.2012.07.008.

[8] J.W. Lee, M.R. Prausnitz, Drug delivery using microneedle patches: not just for skin, Expert Opin. Drug Deliv. 15 (2018) 541-543. https://doi.org/10.1080/17425247.2018.1471059.

[9] I.A. Tekko, T.R. Raj Singh, Microneedles for Ocular Drug Delivery and Targeting: Challenges and Opportunities, Microneedles Drug Vaccine Deliv. Patient Monit. (2018) 283-306. https://doi.org/doi:10.1002/9781119305101.ch10.

[10] L.K. Vora, P.R. Vavia, E. Larrañeta, S.E.J. Bell, R.F. Donnelly, Novel nanosuspension-based dissolving microneedle arrays for transdermal delivery of a hydrophobic drug, J. Interdiscip. Nanomedicine. 3 (2018) 89-101. https://doi.org/10.1002/jin2.41.

[11] E. Larrañeta, L. Vora, Delivery of Nanomedicines Using Microneedles, Microneedles Drug Vaccine Deliv. Patient Monit. https://doi.org/doi:10.1002/9781119305101.ch6.

[12] E. Larrañeta, M.T.C. McCrudden, A.J. Courtenay, R.F. Donnelly, Microneedles: A New Frontier in Nanomedicine Delivery, Pharm. Res. 33 (2016) 1055-1073. https://doi.org/10.1007/s1 1095-016-1885-5.

[13] F.P. Pons-Faudoa, A. Ballerini, J. Sakamoto, A. Grattoni, Advanced implantable drug delivery technologies: transforming the clinical landscape of therapeutics for chronic diseases, Biomed. Microdevices. 21 (2019). https://doi.org/10.1007/s10544-019-0389-6.

[14] L. Rahnfeld, P. Luciani, Injectable lipid-based depot formulations: Where do we stand?, Pharmaceutics. 12 (2020) 1-28. https://doi.org/10.3390/pharmaceutics12060567.

[15] I.A. Tekko, A.D. Permana, L. Vora, T. Hatahet, H.O. McCarthy, R.F. Donnelly, Localised and sustained intradermal delivery of methotrexate using nanocrystal-loaded microneedle arrays: Potential for enhanced treatment of psoriasis, Eur. J. Pharm. Sci. 152 (2020) 105469. https://doi.org/https://doi.org/10.1016/j.ejps.2020.105469.

[16] I.M.N. Hamdan, I.A. Tekko, K.B. Matchett, L.G. Arnaut, C.S. Silva, H.O. McCarthy, R.F. Donnelly, intradermal delivery of a near-infrared photosensitizer using dissolving microneedle arrays, J. Pharm. Sci. 107 (2018) 2439-2450. https://doi.org/10.1016/j.xphs.2018.05.017.

[17] K.M. Ainslie, T.A. Desai, Long Acting Injections and Implants, Long Act. Inject. Implant. (2012) 359-381. https://doi.org/10.1007/978-1-4614-0554-2.

[18] W.Y. Lee, M. Asadujjaman, J.P. Jee, Long acting injectable formulations: the state of the arts and challenges of poly(lactic-co-glycolic acid) microsphere, hydrogel, organogel and liquid crystal, J. Pharm. Investig. 49 (2019) 459-476. https://doi.org/10.1007/s40005-019-00449-9.

[19] U.S. Pharmacopeia, N. Formulary, Effective date 03/30/2020, (2020) 1-36.

[20] L. Bally, H. Thabit, R. Hovorka, Finding the right route for insulin delivery - an overview of implantable pump therapy, Expert Opin. Drug Deliv. 14 (2017) 1103-1111. https://doi.org/10.1080/17425247.2017.1267138.

[21] S. Khan, M.U. Minhas, I.A. Tekko, R.F. Donnelly, R.R.S. Thakur, Evaluation of microneedlesassisted in situ depot forming poloxamer gels for sustained transdermal drug delivery, Drug Deliv. Transl. Res. 9 (2019) 764-782. https://doi.org/10.1007/s13346-019-00617-2.

[22] J. Li, D.J. Mooney, Designing hydrogels for controlled drug delivery, Nat. Rev. Mater. 1 (2016). 
https://doi.org/10.1038/natrevmats.2016.71.

[23] M. Ventre, V. Coppola, M. Iannone, P.A. Netti, I. Tekko, E. Larrañeta, A.M. Rodgers, C.J. Scott, A. Kissenpfennig, R.F. Donnelly, S. Maher, D. Losic, A. George, A. Ramachandran, Nanotechnologies for tissue engineering and regeneration, Nanotechnologies Prev. Regen. Med. (2018) 93-206. https://doi.org/10.1016/B978-0-323-48063-5.00002-2.

[24] K.H. Knight, F.M. Brand, A.S. Mchaourab, G. Veneziano, Implantable intrathecal pumps for chronic pain: highlights and updates, Croat. Med. J. 48 (2007) 22-34. https://pubmed.ncbi.nlm.nih.gov/17309136.

[25] S. Rowlands, S. Searle, Contraceptive implants: current perspectives, Open Access J. Contracept. (2014) 73. https://doi.org/10.2147/oajc.s55968.

[26] K.L. PARKER, R.G. BAENS-BAILON, P.A. LEE, Depot Leuprolide Acetate Dosage for Sexual Precocity*, J. Clin. Endocrinol. Metab. 73 (1991) 50-52. https://doi.org/10.1210/jcem73-1-50.

[27] A.K. Fløgstad, J. Halse, S. Bakke, I. Lancranjan, P. Marbach, C. Bruns, J. Jervell, Sandostatin LAR in acromegalic patients: Long term treatment, J. Clin. Endocrinol. Metab. 82 (1997) 2328. https://doi.org/10.1210/jc.82.1.23.

[28] W. V Bobo, R.C. Shelton, Risperidone long-acting injectable (Risperdal Consta®) for maintenance treatment in patients with bipolar disorder, Expert Rev. Neurother. 10 (2010) 16371658. https://doi.org/10.1586/ern.10.143.

[29] K. Patel, R. Doddapaneni, N. Chowdhury, C.H. Boakye, G. Behl, M. Singh, Tumor stromal disrupting agent enhances the anticancer efficacy of docetaxel loaded PEGylated liposomes in lung cancer, Nanomedicine (Lond). 11 (2016) 1377-1392. https://doi.org/10.2217/nnm.16.37.

[30] J. Monpara, C. Kanthou, G.M. Tozer, P.R. Vavia, Rational Design of Cholesterol Derivative for Improved Stability of Paclitaxel Cationic Liposomes, Pharm. Res. 35 (2018) 90. https://doi.org/10.1007/s1 1095-018-2367-8.

[31] K. Patel, A. Patil, M. Mehta, V. Gota, P. Vavia, Oral delivery of paclitaxel nanocrystal (PNC) with a dual Pgp-CYP3A4 inhibitor: Preparation, characterization and antitumor activity, Int. J. Pharm. 472 (2014) 214-223. https://doi.org/https://doi.org/10.1016/j.ijpharm.2014.06.031.

[32] J.H. Park, M.G. Allen, M.R. Prausnitz, Polymer microneedles for controlled-release drug delivery, Pharm. Res. 23 (2006) 1008-1019. https://doi.org/10.1007/s11095-006-0028-9.

[33] S. Henry, D. V. McAllister, M.G. Allen, M.R. Prausnitz, Microfabricated microneedles: A novel approach to transdermal drug delivery, J. Pharm. Sci. 87 (1998) 922-925. https://doi.org/10.1021/js980042+.

[34] R.F. Donnelly, E. Larrañeta, Microarray patches: potentially useful delivery systems for longacting nanosuspensions, Drug Discov. Today. 23 (2018) 1026-1033. https://doi.org/10.1016/j.drudis.2017.10.013.

[35] J.W. Lee, J.-H. Park, M.R. Prausnitz, Dissolving microneedles for transdermal drug delivery., Biomaterials. (2008). https://doi.org/10.1016/j.biomaterials.2007.12.048.

[36] E. Larrañeta, R.E.M. Lutton, A.D. Woolfson, R.F. Donnelly, Microneedle arrays as transdermal and intradermal drug delivery systems: Materials science, manufacture and commercial development, Mater. Sci. Eng. R Reports. $104 \quad$ (2016) 1-32. https://doi.org/https://doi.org/10.1016/j.mser.2016.03.001.

[37] R. Jamaledin, C. Di Natale, V. Onesto, Z. Taraghdari, E. Zare, P. Makvandi, R. Vecchione, P. Netti, Progress in Microneedle-Mediated Protein Delivery, J. Clin. Med. 9 (2020) 542. https://doi.org/10.3390/jcm9020542.

[38] L.K. Vora, P.R. Vavia, E. Larrañeta, S.E.J. Bell, R.F. Donnelly, Novel nanosuspension-based dissolving microneedle arrays for transdermal delivery of a hydrophobic drug, J. Interdiscip. Nanomedicine. 3 (2018) 89-101. https://doi.org/10.1002/jin2.41.

[39] L.K. Vora, R.F. Donnelly, E. Larrañeta, P. González-Vázquez, R.R.S. Thakur, P.R. Vavia, Novel bilayer dissolving microneedle arrays with concentrated PLGA nano-microparticles for targeted intradermal delivery: Proof of concept., J. Control. Release. 265 (2017) 93-101. https://doi.org/10.1016/j.jconrel.2017.10.005.

[40] L.K. Vora, P.R. Vavia, E. Larrañeta, S.E.J. Bell, R.F. Donnelly, Novel nanosuspension-based dissolving microneedle arrays for transdermal delivery of a hydrophobic drug, J. Interdiscip. Nanomedicine. 3 (2018) 89-101. https://doi.org/10.1002/jin2.41. 
[41] S.G. Lee, J.H. Jeong, K.M. Lee, K.H. Jeong, H. Yang, M. Kim, H. Jung, S. Lee, Y.W. Choi, Nanostructured lipid carrier-loaded hyaluronic acid microneedles for controlled dermal delivery of a lipophilic molecule, Int. J. Nanomedicine. 9 (2013) 289-299. https://doi.org/10.2147/IJN.S54529.

[42] M. Chen, G. Quan, Y. Sun, D. Yang, X. Pan, C. Wu, Nanoparticles-encapsulated polymeric microneedles for transdermal drug delivery, J. Control. Release. 325 (2020) 163-175. https://doi.org/https://doi.org/10.1016/j.jconrel.2020.06.039.

[43] J. Kennedy, E. Larrañeta, M.T.C. McCrudden, C.M. McCrudden, A.J. Brady, S.J. Fallows, H.O. McCarthy, A. Kissenpfennig, R.F. Donnelly, In vivo studies investigating biodistribution of nanoparticle-encapsulated rhodamine B delivered via dissolving microneedles, J. Control. Release. 265 (2017) 57-65. https://doi.org/https://doi.org/10.1016/j.jconrel.2017.04.022.

[44] C.S.J. Campbell, L.R. Contreras-Rojas, M.B. Delgado-Charro, R.H. Guy, Objective assessment of nanoparticle disposition in mammalian skin after topical exposure, J. Control. Release. 162 (2012) 201-207. https://doi.org/https://doi.org/10.1016/j.jconrel.2012.06.024.

[45] W. Li, R.N. Terry, J. Tang, M.R. Feng, S.P. Schwendeman, M.R. Prausnitz, Rapidly separable microneedle patch for the sustained release of a contraceptive, Nat. Biomed. Eng. 3 (2019) 220229. https://doi.org/10.1038/s41551-018-0337-4.

[46] Y.J. Lee, S.H. Park, S. Il Kim, K.J. Lee, W.H. Ryu, Rapidly Detachable Microneedles Using Porous Water-Soluble Layer for Ocular Drug Delivery, Adv. Mater. Technol. 5 (2020) 1901145. https://doi.org/10.1002/admt.201901145.

[47] W. Li, J. Tang, R.N. Terry, S. Li, A. Brunie, R.L. Callahan, R.K. Noel, C.A. Rodrl'liguez, S.P. Schwendeman, M.R. Prausnitz, Long-acting reversible contraception by effervescent microneedle patch, Sci. Adv. 5 (2019). https://doi.org/10.1126/sciadv.aaw8145.

[48] M.J. Kim, S.C. Park, B. Rizal, G. Guanes, S.K. Baek, J.H. Park, A.R. Betz, S.O. Choi, Fabrication of circular obelisk-type multilayer microneedles using micro-milling and spray deposition, Front. Bioeng. Biotechnol. 6 (2018) 54. https://doi.org/10.3389/fbioe.2018.00054.

[49] A. Than, C. Liu, H. Chang, P.K. Duong, C.M.G. Cheung, C. Xu, X. Wang, P. Chen, Selfimplantable double-layered micro-drug-reservoirs for efficient and controlled ocular drug delivery, Nat. Commun. 9 (2018) 1-12. https://doi.org/10.1038/s41467-018-06981-w.

[50] P.E. Daddona, J.A. Matriano, J. Mandema, Y.-F. Maa, Parathyroid Hormone (1-34)-Coated Microneedle Patch System: Clinical Pharmacokinetics and Pharmacodynamics for Treatment of Osteoporosis, Pharm. Res. 28 (2011) 159-165. https://doi.org/10.1007/s11095-010-0192-9.

[51] M. Cormier, B. Johnson, M. Ameri, K. Nyam, L. Libiran, D.D. Zhang, P. Daddona, Transdermal delivery of desmopressin using a coated microneedle array patch system, J. Control. Release. 97 (2004) 503-511. https://doi.org/https://doi.org/10.1016/j.jconrel.2004.04.003.

[52] C. Tas, S. Mansoor, H. Kalluri, V.G. Zarnitsyn, S.-O. Choi, A.K. Banga, M.R. Prausnitz, Delivery of salmon calcitonin using a microneedle patch, Int. J. Pharm. 423 (2012) 257-263. https://doi.org/https://doi.org/10.1016/j.ijpharm.2011.11.046.

[53] M. Pearton, V. Saller, S.A. Coulman, C. Gateley, A. V Anstey, V. Zarnitsyn, J.C. Birchall, Microneedle delivery of plasmid DNA to living human skin: Formulation coating, skin insertion and gene expression, J. Control. Release. $160 \quad$ (2012) 561-569. https://doi.org/https://doi.org/10.1016/j.jconrel.2012.04.005.

[54] T.M. Tuan-Mahmood, M.T.C. McCrudden, B.M. Torrisi, E. McAlister, M.J. Garland, T.R.R. Singh, R.F. Donnelly, Microneedles for intradermal and transdermal drug delivery, Eur. J. Pharm. Sci. 50 (2013) 623-637. https://doi.org/10.1016/j.ejps.2013.05.005.

[55] J. Yang, X. Liu, Y. Fu, Y. Song, Recent advances of microneedles for biomedical applications: drug delivery and beyond, Acta Pharm. Sin. B. 9 (2019) 469-483. https://doi.org/https://doi.org/10.1016/j.apsb.2019.03.007.

[56] H.J.G.E. Gardeniers, R. Luttge, E.J.W. Berenschot, M.J. De Boer, S.Y. Yeshurun, M. Hefetz, R. Van't Oever, A. Van Den Berg, Silicon micromachined hollow microneedles for transdermal liquid transport, J. Microelectromechanical Syst. $12 \quad$ (2003) 855-862. https://doi.org/10.1109/JMEMS.2003.820293.

[57] R.F. Donnelly, M.T.C. McCrudden, A.Z. Alkilani, E. Larrañeta, E. McAlister, A.J. Courtenay, M.C. Kearney, T.R. Raj Singh, H.O. McCarthy, V.L. Kett, E. Caffarel-Salvador, S. Al-Zahrani, A.D. Woolfson, Hydrogel-forming microneedles prepared from "super swelling" polymers 
combined with lyophilised wafers for transdermal drug delivery, PLoS One. 9 (2014). https://doi.org/10.1371/journal.pone.0111547.

[58] R.F. Donnelly, T.R.R. Singh, M.J. Garland, K. Migalska, R. Majithiya, C.M. McCrudden, P.L. Kole, T.M.T. Mahmood, H.O. McCarthy, A.D. Woolfson, Hydrogel-forming microneedle arrays for enhanced transdermal drug delivery, Adv. Funct. Mater. (2012). https://doi.org/10.1002/adfm.201200864.

[59] Y.K. Demir, A.Ü. Metin, B. Şatıroğlu, M.E. Solmaz, V. Kayser, K. Mäder, Poly (methyl vinyl ether-co-maleic acid) - Pectin based hydrogel-forming systems: Gel, film, and microneedles, Eur. J. Pharm. Biopharm. 117 (2017) 182-194. https://doi.org/10.1016/j.ejpb.2017.04.018.

[60] E. Larrañeta, L. Barturen, M. Ervine, R.F. Donnelly, Hydrogels based on poly(methyl vinyl ether-co-maleic acid) and Tween 85 for sustained delivery of hydrophobic drugs, Int. J. Pharm. 538 (2018) 147-158. https://doi.org/10.1016/j.ijpharm.2018.01.025.

[61] E. Larrañeta, M. Henry, N.J. Irwin, J. Trotter, A.A. Perminova, R.F. Donnelly, Synthesis and characterization of hyaluronic acid hydrogels crosslinked using a solvent-free process for potential biomedical applications, Carbohydr. Polym. 181 (2018) 1194-1205. https://doi.org/10.1016/j.carbpol.2017.12.015.

[62] Y.K. Demir, Z. Akan, O. Kerimoglu, Characterization of polymeric microneedle arrays for transdermal drug delivery, PLoS One. 8 (2013) e77289. https://doi.org/10.1371/journal.pone.0077289.

[63] I.A. Tekko, G. Chen, J. Domínguez-Robles, R.R.S. Thakur, I.M.N. Hamdan, L. Vora, E. Larrañeta, J.C. McElnay, H.O. McCarthy, M. Rooney, R.F. Donnelly, Development and characterisation of novel poly (vinyl alcohol)/poly (vinyl pyrrolidone)-based hydrogel-forming microneedle arrays for enhanced and sustained transdermal delivery of methotrexate, Int. J. Pharm. 586 (2020) 119580. https://doi.org/10.1016/j.ijpharm.2020.119580.

[64] E.M. Migdadi, A.J. Courtenay, I.A. Tekko, M.T.C. Mccrudden, M. Kearney, E. Mcalister, H.O. Mccarthy, R.F. Donnelly, Hydrogel-forming microneedles enhance transdermal delivery of metformin hydrochloride, J. Control. Release. 285 (2018) 142-151. https://doi.org/10.1016/j.jconrel.2018.07.009.

[65] R.F. Donnelly, M.T.C. McCrudden, A. Zaid Alkilani, E. Larrañeta, E. McAlister, A.J. Courtenay, M.-C. Kearney, T.R.R. Singh, H.O. McCarthy, V.L. Kett, E. Caffarel-Salvador, S. Al-Zahrani, A.D. Woolfson, Hydrogel-Forming Microneedles Prepared from "Super Swelling" Polymers Combined with Lyophilised Wafers for Transdermal Drug Delivery, PLoS One. 9 (2014). https://dx.plos.org/10.1371/journal.pone.0111547.

[66] A.J. Courtenay, M.T.C. McCrudden, K.J. McAvoy, H.O. McCarthy, R.F. Donnelly, Microneedle-mediated transdermal delivery of bevacizumab, Mol. Pharm. 15 (2018) 35453556. https://doi.org/10.1021/acs.molpharmaceut.8b00544.

[67] C. Plamadeala, S.R. Gosain, F. Hischen, B. Buchroithner, S. Puthukodan, J. Jacak, A. Bocchino, D. Whelan, C. O'Mahony, W. Baumgartner, J. Heitz, Bio-inspired microneedle design for efficient drug/vaccine coating, Biomed. Microdevices. 22 (2020) 1-9. https://doi.org/10.1007/s10544-019-0456-z.

[68] M.K. Ramasubramanian, O.M. Barham, V. Swaminathan, Mechanics of a mosquito bite with applications to microneedle design., Bioinspir. Biomim. 3 (2008) 46001. https://doi.org/10.1088/1748-3182/3/4/046001.

[69] M. Suzuki, T. Sawa, T. Takahashi, S. Aoyagi, Ultrafine three-dimensional (3D) laser lithographic fabrication of microneedle and its application to painless insertion and blood sampling inspired by mosquito, in: 2015 IEEE/RSJ Int. Conf. Intell. Robot. Syst., 2015: pp. 2748-2753. https://doi.org/10.1109/IROS.2015.7353754.

[70] K. Oka, S. Aoyagi, Y. Arai, Y. Isono, G. Hashiguchi, H. Fujita, Fabrication of a micro needle for a trace blood test, Sensors Actuators A Phys. 97-98 (2002) 478-485. https://doi.org/https://doi.org/10.1016/S0924-4247(01)00872-X.

[71] S.Y. Yang, E.D. O’Cearbhaill, G.C. Sisk, K.M. Park, W.K. Cho, M. Villiger, B.E. Bouma, B. Pomahac, J.M. Karp, A bio-inspired swellable microneedle adhesive for mechanical interlocking with tissue., Nat. Commun. 4 (2013) 1702. https://doi.org/10.1038/ncomms2715.

[72] M.S. Gerstel, V.A. Place, Drug delivery device - US 3964482 A. U. S. Pat. 482 (1976).

[73] M.R. Prausnitz, Microneedles for transdermal drug delivery, Adv. Drug Deliv. Rev. 56 (2004) 
581-587. https://doi.org/10.1016/j.addr.2003.10.023.

[74] A.S. Cordeiro, I.A. Tekko, M.H. Jomaa, L. Vora, E. McAlister, F. Volpe-Zanutto, M. Nethery, P.T. Baine, N. Mitchell, D.W. McNeill, R.F. Donnelly, Two-Photon Polymerisation 3D Printing of Microneedle Array Templates with Versatile Designs: Application in the Development of Polymeric Drug Delivery Systems, Pharm. Res. 37 (2020) 174. https://doi.org/10.1007/s11095020-02887-9.

[75] F. Pérennès, B. Marmiroli, M. Matteucci, M. Tormen, L. Vaccari, E. Di Fabrizio, Sharp beveled tip hollow microneedle arrays fabricated by LIGA and 3D soft lithography with polyvinyl alcohol, J. Micromechanics Microengineering. $16 \quad$ (2006) 473-479. https://doi.org/10.1088/0960-1317/16/3/001.

[76] J.M. Lippmann, E.J. Geiger, A.P. Pisano, Polymer investment molding: Method for fabricating hollow, microscale parts, Sensors Actuators, A Phys. 134 (2007) 2-10. https://doi.org/10.1016/j.sna.2006.05.009.

[77] K. Lee, S.H. Park, J. Lee, S. Ryu, C. Joo, W. Ryu, Three-step thermal drawing for rapid prototyping of highly customizable microneedles for vascular tissue insertion, Pharmaceutics. 11 (2019). https://doi.org/10.3390/pharmaceutics11030100.

[78] Y.A. Gomaa, D.I.J. Morrow, M.J. Garland, R.F. Donnelly, L.K. El-Khordagui, V.M. Meidan, Effects of microneedle length, density, insertion time and multiple applications on human skin barrier function: assessments by transepidermal water loss., Toxicol. In Vitro. 24 (2010) 19711978. https://doi.org/10.1016/j.tiv.2010.08.012.

[79] K. Badnikar, S.N. Jayadevi, S. Pahal, S. Sripada, M.M. Nayak, P.K. Vemula, D.N. Subrahmanyam, Generic Molding Platform for Simple, Low-Cost Fabrication of Polymeric Microneedles, Macromol. Mater. Eng. $305 \quad$ (2020) 2000072. https://doi.org/10.1002/mame.202000072.

[80] Y. Park, J. Park, G.S. Chu, K.S. Kim, J.H. Sung, B. Kim, Transdermal delivery of cosmetic ingredients using dissolving polymer microneedle arrays, Biotechnol. Bioprocess Eng. 20 (2015) 543-549. https://doi.org/10.1007/s12257-014-0775-0.

[81] M.C. Chen, S.F. Huang, K.Y. Lai, M.H. Ling, Fully embeddable chitosan microneedles as a sustained release depot for intradermal vaccination, Biomaterials. 34 (2013) 3077-3086. https://doi.org/10.1016/j.biomaterials.2012.12.041.

[82] R. Haj-Ahmad, H. Khan, M.S. Arshad, M. Rasekh, A. Hussain, S. Walsh, X. Li, M.-W. Chang, Z. Ahmad, Microneedle Coating Techniques for Transdermal Drug Delivery., Pharmaceutics. 7 (2015) 486-502. https://doi.org/10.3390/pharmaceutics7040486.

[83] H.S. Gill, M.R. Prausnitz, Coated microneedles for transdermal delivery, J. Control. Release. 117 (2007) 227-237. https://doi.org/https://doi.org/10.1016/j.jconrel.2006.10.017.

[84] M.G. McGrath, A. Vrdoljak, C. O’Mahony, J.C. Oliveira, A.C. Moore, A.M. Crean, Determination of parameters for successful spray coating of silicon microneedle arrays, Int. J. Pharm. 415 (2011) 140-149. https://doi.org/10.1016/j.ijpharm.2011.05.064.

[85] X. Chen, T.W. Prow, M.L. Crichton, D.W.K. Jenkins, M.S. Roberts, I.H. Frazer, G.J.P. Fernando, M.A.F. Kendall, Dry-coated microprojection array patches for targeted delivery of immunotherapeutics to the skin, J. Control. Release. 139 (2009) 212-220. https://doi.org/https://doi.org/10.1016/j.jconrel.2009.06.029.

[86] R.D. Boehm, P.R. Miller, S.L. Hayes, N.A. Monteiro-Riviere, R.J. Narayan, Modification of microneedles using inkjet printing, AIP Adv. 1 (2011) 1-13. https://doi.org/10.1063/1.3602461.

[87] S. Ross, N. Scoutaris, D. Lamprou, D. Mallinson, D. Douroumis, Inkjet printing of insulin microneedles for transdermal delivery., Drug Deliv. Transl. Res. 5 (2015) 451-461. https://doi.org/10.1007/s13346-015-0251-1.

[88] R. Haj-Ahmad, M. Rasekh, K. Nazari, Y. Li, Y. Fu, B. Li, Q. Zhang, Z. Xia, H. Liu, T. Gu, G. Wang, I. Kucuk, H. Khan, M.S. Arshad, X.L. and Z. Ahmad, EHDA Spraying: A Multi-Material Nano-Engineering Route, Curr. Pharm. Des. 21 (2015) 3239-3247. https://doi.org/http://dx.doi.org/10.2174/1381612821666150531171506.

[89] M.A. Invernale, B.C. Tang, R.L. York, L. Le, D.Y. Hou, D.G. Anderson, Microneedle Electrodes Toward an Amperometric Glucose-Sensing Smart Patch, Adv. Healthc. Mater. 3 (2014) 338-342. https://doi.org/10.1002/adhm.201300142.

[90] Y.-C. Kim, F.-S. Quan, R.W. Compans, S.-M. Kang, M.R. Prausnitz, Formulation and coating 
of microneedles with inactivated influenza virus to improve vaccine stability and immunogenicity, J. Control. Release. 142 (2010) 187-195. https://doi.org/10.1016/j.jconrel.2009.10.013.

[91] S.P. Sullivan, D.G. Koutsonanos, M. Del Pilar Martin, J.W. Lee, V. Zarnitsyn, S.-O. Choi, N. Murthy, R.W. Compans, I. Skountzou, M.R. Prausnitz, Dissolving polymer microneedle patches for influenza vaccination, Nat. Med. 16 (2010) 915-920. https://doi.org/10.1038/nm.2182.

[92] P. Zhang, C. Dalton, G.A. Jullien, Design and fabrication of MEMS-based microneedle arrays for medical applications, Microsyst. Technol. $15 \quad$ (2009) 1073-1082. https://doi.org/10.1007/s00542-009-0883-5.

[93] K. Lee, H.C. Lee, D.-S. Lee, H. Jung, Drawing Lithography: Three-Dimensional Fabrication of an Ultrahigh-Aspect-Ratio Microneedle, Adv. Mater. 22 (2010) 483-486. https://doi.org/https://doi.org/10.1002/adma.200902418.

[94] C.G. Li, C.Y. Lee, K. Lee, H. Jung, An optimized hollow microneedle for minimally invasive blood extraction, Biomed. Microdevices. 15 (2013) 17-25. https://doi.org/10.1007/s10544-0129683-2.

[95] K. Kim, J.-B. Lee, High aspect ratio tapered hollow metallic microneedle arrays with microfluidic interconnector, Microsyst. Technol. 13 (2007) 231-235. https://doi.org/10.1007/s00542-006-0221-0.

[96] Y. Li, H. Zhang, R. Yang, Y. Laffitte, U. Schmill, W. Hu, M. Kaddoura, E.J.M. Blondeel, B. Cui, Fabrication of sharp silicon hollow microneedles by deep-reactive ion etching towards minimally invasive diagnostics, Microsystems Nanoeng. 5 (2019). https://doi.org/10.1038/s41378-019-0077-y.

[97] J. Domínguez-Robles, C. Mancinelli, E. Mancuso, I. García-Romero, B.F. Gilmore, L. Casettari, E. Larrañeta, D.A. Lamprou, 3D printing of drug-loaded thermoplastic polyurethane meshes: A potential material for soft tissue reinforcement in vaginal surgery, Pharmaceutics. 12 (2020). https://doi.org/10.3390/pharmaceutics12010063.

[98] S.N. Economidou, C.P.P. Pere, A. Reid, M.J. Uddin, J.F.C. Windmill, D.A. Lamprou, D. Douroumis, 3D printed microneedle patches using stereolithography (SLA)for intradermal insulin delivery, Mater. Sci. Eng. C. 102 (2019) 743-755. https://doi.org/10.1016/j.msec.2019.04.063.

[99] Z. Chen, J. He, J. Qi, Q. Zhu, W. Wu, Y. Lu, Long-acting microneedles: a progress report of the state-of-the-art techniques, Drug Discov. Today. $25 \quad$ (2020) 1462-1468. https://doi.org/https://doi.org/10.1016/j.drudis.2020.05.006.

[100] M.-C. Chen, S.-F. Huang, K.-Y. Lai, M.-H. Ling, Fully embeddable chitosan microneedles as a sustained release depot for intradermal vaccination, Biomaterials. 34 (2013) 3077-3086. https://doi.org/https://doi.org/10.1016/j.biomaterials.2012.12.041.

[101] X. Hong, L. Wei, F. Wu, Z. Wu, L. Chen, Z. Liu, W. Yuan, Dissolving and biodegradable microneedle technologies for transdermal sustained delivery of drug and vaccine, Drug Des. Devel. Ther. 7 (2013) 945-952. https://doi.org/10.2147/DDDT.S44401.

[102] E. Larrañeta, R.E.M. Lutton, A.D. Woolfson, R.F. Donnelly, Microneedle arrays as transdermal and intradermal drug delivery systems: Materials science, manufacture and commercial development, Mater. Sci. Eng. R Reports. $104 \quad$ (2016) 1-32. https://doi.org/10.1016/j.mser.2016.03.001.

[103] J.G. Hardy, E. Larrañeta, R.F. Donnelly, N. McGoldrick, K. Migalska, M.T.C. McCrudden, N.J. Irwin, L. Donnelly, C.P. McCoy, Hydrogel-Forming Microneedle Arrays Made from LightResponsive Materials for On-Demand Transdermal Drug Delivery, Mol. Pharm. 13 (2016) 907914. https://doi.org/10.1021/acs.molpharmaceut.5b00807.

[104] I. Tekko, L. Vora, M. McCrudden, C. Jarrahian, A. Rein-Weston, D. Zehrung, P. Giffen, H. McCarthy, R. Donnelly, Novel dissolving bilayer microarray patches as a minimally invasive, efficient intradermal delivery system for a long-acting cabotegravir nanosuspension, (2019).

[105] A.S. Cordeiro, I.A. Tekko, M.H. Jomaa, L. Vora, E. McAlister, F. Volpe-Zanutto, M. Nethery, P.T. Baine, N. Mitchell, D.W. McNeill, R.F. Donnelly, Two-Photon Polymerisation 3D Printing of Microneedle Array Templates with Versatile Designs: Application in the Development of Polymeric Drug Delivery Systems, Pharm. Res. 37 (2020) 1-15. https://doi.org/10.1007/s11095-020-02887-9. 
[106] M. Wang, L. Hu, C. Xu, Recent advances in the design of polymeric microneedles for transdermal drug delivery and biosensing, Lab Chip. 17 (2017) 1373-1387. https://doi.org/10.1039/C7LC00016B.

[107] F.J. Verbaan, S.M. Bal, D.J. van den Berg, J.A. Dijksman, M. van Hecke, H. Verpoorten, A. van den Berg, R. Luttge, J.A. Bouwstra, Improved piercing of microneedle arrays in dermatomed human skin by an impact insertion method, J. Control. Release. 128 (2008) 80-88. https://doi.org/10.1016/j.jconrel.2008.02.009.

[108] J.H. Park, M.G. Allen, M.R. Prausnitz, Biodegradable polymer microneedles: Fabrication, mechanics and transdermal drug delivery, J. Control. Release. 104 (2005) 51-66. https://doi.org/10.1016/j.jconrel.2005.02.002.

[109] M.C. Chen, M.H. Ling, K.Y. Lai, E. Pramudityo, Chitosan microneedle patches for sustained transdermal delivery of macromolecules, Biomacromolecules. 13 (2012) 4022-4031. https://doi.org/10.1021/bm301293d.

[110] S.D. Gittard, A. Ovsianikov, N.A. Monteiro-Riviere, J. Lusk, P. Morel, P. Minghetti, C. Lenardi, B.N. Chichkov, R.J. Narayan, Fabrication of polymer microneedles using a two-photon polymerization and micromolding process, J. Diabetes Sci. Technol. 3 (2009) 304-311. https://doi.org/10.1177/193229680900300211.

[111] I.K. Ramöller, E. McAlister, A. Bogan, A.S. Cordeiro, R.F. Donnelly, Novel design approaches in the fabrication of polymeric microarray patches via micromoulding, Micromachines. 11 (2020) 554. https://doi.org/10.3390/MI11060554.

[112] E. Larrañeta, J. Moore, E.M. Vicente-Pérez, P. González-Vázquez, R. Lutton, A.D. Woolfson, R.F. Donnelly, A proposed model membrane and test method for microneedle insertion studies., Int. J. Pharm. (2014). https://doi.org/10.1016/j.ijpharm.2014.05.042.

[113] J.S. Kochhar, T.C. Quek, W.J. Soon, J. Choi, S. Zou, L. Kang, Effect of microneedle geometry and supporting substrate on microneedle array penetration into skin, J. Pharm. Sci. 102 (2013) 4100-4108. https://doi.org/10.1002/jps.23724.

[114] A.D. Permana, A.J. Paredes, F. Volpe-Zanutto, Q.K. Anjani, E. Utomo, R.F. Donnelly, Dissolving microneedle-mediated dermal delivery of itraconazole nanocrystals for improved treatment of cutaneous candidiasis, Eur. J. Pharm. Biopharm. 154 (2020) 50-61. https://doi.org/10.1016/j.ejpb.2020.06.025.

[115] X. Zhao, S. Zhang, G. Yang, Z. Zhou, Y. Gao, Exploring Trehalose on the Release of Levonorgestrel from Implantable PLGA Microneedles, Polymers (Basel). 12 (2020) 59. https://doi.org/10.3390/polym12010059.

[116] M. He, G. Yang, X. Zhao, S. Zhang, Y. Gao, Intradermal Implantable PLGA Microneedles for Etonogestrel Sustained Release, J. Pharm. Sci. (2020) 1-9. https://doi.org/10.1016/j.xphs.2020.02.009.

[117] A.D. Permana, M. Mir, E. Utomo, R.F. Donnelly, Bacterially sensitive nanoparticle-based dissolving microneedles of doxycycline for enhanced treatment of bacterial biofilm skin infection: A proof of concept study, Int. J. Pharm. X. 2 (2020) 100047. https://doi.org/https://doi.org/10.1016/j.ijpx.2020.100047.

[118] M.T.C. Mc Crudden, E. Larrañeta, A. Clark, C. Jarrahian, A. Rein-Weston, S. Lachau-Durand, N. Niemeijer, P. Williams, C. Haeck, H.O. McCarthy, D. Zehrung, R.F. Donnelly, Design, formulation and evaluation of novel dissolving microarray patches containing a long-acting rilpivirine nanosuspension, J. Control. Release. $292 \quad$ (2018) 119-129. https://doi.org/https://doi.org/10.1016/j.jconrel.2018.11.002.

[119] S. Abdelghany, I.A. Tekko, L. Vora, E. Larrañeta, A.D. Permana, R.F. Donnelly, Nanosuspension-Based Dissolving Microneedle Arrays for Intradermal Delivery of Curcumin., Pharmaceutics. 11 (2019). https://doi.org/10.3390/pharmaceutics11070308.

[120] B. Yavuz, L. Chambre, K. Harrington, J. Kluge, L. Valenti, D.L. Kaplan, Silk Fibroin Microneedle Patches for the Sustained Release of Levonorgestrel, ACS Appl. Bio Mater. 3 (2020) 5375-5382. https://doi.org/10.1021/acsabm.0c00671.

[121] Y.H. Chiu, M.C. Chen, S.W. Wan, Sodium Hyaluronate/Chitosan Composite Microneedles as a Single-Dose Intradermal Immunization System, Biomacromolecules. 19 (2018) 2278-2285. https://doi.org/10.1021/acs.biomac.8b00441.

[122] M.-C. Chen, K.-Y. Lai, M.-H. Ling, C.-W. Lin, Enhancing immunogenicity of antigens through 
sustained intradermal delivery using chitosan microneedles with a patch-dissolvable design, Acta Biomater. 65 (2018) 66-75. https://doi.org/https://doi.org/10.1016/j.actbio.2017.11.004.

[123] M.-C. Chen, M.-H. Ling, K.-Y. Lai, E. Pramudityo, Chitosan Microneedle Patches for Sustained Transdermal Delivery of Macromolecules, Biomacromolecules. 13 (2012) 4022-4031. https://doi.org/10.1021/bm301293d.

[124] D.S. Kim, J.T. Choi, C.B. Kim, Y.R. Shin, P. gu Park, H. Kim, J.M. Lee, J.H. Park, Microneedle Array Patch (MAP) Consisting of Crosslinked Hyaluronic Acid Nanoparticles for Processability and Sustained Release, Pharm. Res. 37 (2020). https://doi.org/10.1007/s11095-020-2768-3.

[125] S. Wang, M. Zhu, L. Zhao, D. Kuang, S.C. Kundu, S. Lu, Insulin-Loaded Silk Fibroin Microneedles as Sustained Release System, ACS Biomater. Sci. Eng. 5 (2019) 1887-1894. https://doi.org/10.1021/acsbiomaterials.9b00229.

[126] X. Lan, J. She, D.-A. Lin, Y. Xu, X. Li, W.-F. Yang, V.W.Y. Lui, L. Jin, X. Xie, Y.-X. Su, Microneedle-Mediated Delivery of Lipid-Coated Cisplatin Nanoparticles for Efficient and Safe Cancer Therapy., ACS Appl. Mater. Interfaces. $10 \quad$ (2018) 33060-33069. https://doi.org/10.1021/acsami.8b12926.

[127] A.D. Permana, I. Tekko, M. McCrudden, Q.K. Anjani, D. Ramadon, H. McCarthy, R. Donnelly, Solid lipid nanoparticle-based dissolving microneedles: A promising intradermal lymph targeting drug delivery system with potential for enhanced treatment of lymphatic filariasis, J. Control. Release. 316 (2019). https://doi.org/10.1016/j.jconrel.2019.10.004.

[128] M.-C. Kearney, P.E. McKenna, H.L. Quinn, A.J. Courtenay, E. Larrañeta, R.F. Donnelly, Design and Development of Liquid Drug Reservoirs for Microneedle Delivery of Poorly Soluble $\begin{array}{lllll}\text { Drug } & \text { Molecules, } & \text { Pharmaceutics. } & 11 & \text { (2019) }\end{array}$ https://doi.org/10.3390/pharmaceutics11110605.

[129] I.A. Tekko, G. Chen, J. Domínguez-Robles, R.R.S. Thakur, I.M.N. Hamdan, L. Vora, E. Larrañeta, J.C. McElnay, H.O. McCarthy, M. Rooney, R.F. Donnelly, Development and characterisation of novel poly (vinyl alcohol)/poly (vinyl pyrrolidone)-based hydrogel-forming microneedle arrays for enhanced and sustained transdermal delivery of methotrexate, Int. J. Pharm. 586 (2020) 119580. https://doi.org/https://doi.org/10.1016/j.ijpharm.2020.119580.

[130] Y. Zhang, D. Wang, M. Gao, B. Xu, J. Zhu, W. Yu, D. Liu, G. Jiang, Separable Microneedles for Near-Infrared Light-Triggered Transdermal Delivery of Metformin in Diabetic Rats, ACS Biomater. Sci. Eng. 4 (2018) 2879-2888. https://doi.org/10.1021/acsbiomaterials.8b00642.

[131] K. Tsioris, W.K. Raja, E.M. Pritchard, B. Panilaitis, D.L. Kaplan, F.G. Omenetto, Fabrication of silk microneedles for controlled-release drug delivery, Adv. Funct. Mater. 22 (2012) 330335. https://doi.org/10.1002/adfm.201102012.

[132] J. Yu, J. Wang, Y. Zhang, G. Chen, W. Mao, Y. Ye, A.R. Kahkoska, J.B. Buse, R. Langer, Z. $\mathrm{Gu}$, Glucose-responsive insulin patch for the regulation of blood glucose in mice and minipigs, Nat. Biomed. Eng. 4 (2020) 499-506. https://doi.org/10.1038/s41551-019-0508-y.

[133] G. Du, X. Sun, Current Advances in Sustained Release Microneedles, Pharm. Front. 02 (2020) e11-e22. https://doi.org/10.1055/s-0040-1701435.

[134] S. Kutlehria, G. Behl, K. Patel, R. Doddapaneni, I. Vhora, N. Chowdhury, A. Bagde, M. Singh, Cholecalciferol-PEG Conjugate Based Nanomicelles of Doxorubicin for Treatment of TripleNegative Breast Cancer, AAPS PharmSciTech. $19 \quad$ (2018) 792-802. https://doi.org/10.1208/s12249-017-0885-z.

[135] J. V Natarajan, C. Nugraha, X.W. Ng, S. Venkatraman, Sustained-release from nanocarriers: a $\begin{array}{llllll}\text { review, J. } & \text { Control. } & \text { Release. } & 193 & \text { (2014) } & \text { 122-138. }\end{array}$ https://doi.org/https://doi.org/10.1016/j.jconrel.2014.05.029.

[136] A.D. Bangham, R.W. Horne, Negative staining of phospholipids and their structural modification by surface-active agents as observed in the electron microscope, J. Mol. Biol. 8 (1964) 660-668. https://doi.org/10.1016/S0022-2836(64)80115-7.

[137] V. Weissig, Liposomes came first: The early history of liposomology, in: G. D'Souza (Ed.), Liposomes. Methods Mol. Biol. Vol 1522, Humana Press, New York, 2017. https://doi.org/10.1007/978-1-4939-6591-5_1.

[138] P. Karunanidhi, P. Ekambaram, A. Abdul, H. Sathali, K. Priyanka, Solid lipid nanoparticles: A review, Sci. Revs. Chem. Commun. 2 (2012) 80-102.

[139] S. Weber, A. Zimmer, J. Pardeike, Solid Lipid Nanoparticles (SLN) and Nanostructured Lipid 
Carriers (NLC) for pulmonary application: A review of the state of the art, Eur. J. Pharm. Biopharm. 86 (2014) 7-22. https://doi.org/10.1016/j.ejpb.2013.08.013.

[140] R.H. Müller, K. Mäder, S. Gohla, Solid lipid nanoparticles (SLN) for controlled drug delivery A review of the state of the art, Eur. J. Pharm. Biopharm. 50 (2000) 161-177. https://doi.org/10.1016/S0939-6411(00)00087-4.

[141] A. Garcês, M.H. Amaral, J.M. Sousa Lobo, A.C. Silva, Formulations based on solid lipid nanoparticles (SLN) and nanostructured lipid carriers (NLC) for cutaneous use: A review, Eur. J. Pharm. Sci. 112 (2018) 159-167. https://doi.org/10.1016/j.ejps.2017.11.023.

[142] Y. Qiu, Y. Gao, K. Hu, F. Li, Enhancement of skin permeation of docetaxel: A novel approach combining microneedle and elastic liposomes, J. Control. Release. 129 (2008) 144-150. https://doi.org/10.1016/j.jconrel.2008.04.019.

[143] G. Chen, B. Hao, D. Ju, M. Liu, H. Zhao, Z. Du, J. Xia, Pharmacokinetic and pharmacodynamic study of triptolide-loaded liposome hydrogel patch under microneedles on rats with collageninduced arthritis, Acta Pharm. Sin. B. 5 (2015) 569-576. https://doi.org/10.1016/j.apsb.2015.09.006.

[144] S.G. Lee, J.H. Jeong, K.M. Lee, K.H. Jeong, H. Yang, M. Kim, H. Jung, S. Lee, Y.W. Choi, Nanostructured lipid carrier-loaded hyaluronic acid microneedles for controlled dermal delivery of a lipophilic molecule, Int. J. Nanomedicine. 9 (2013) 289-299. https://doi.org/10.2147/IJN.S54529.

[145] T. Guo, N. Cheng, J. Zhao, X. Hou, Y. Zhang, N. Feng, Novel nanostructured lipid carriersloaded dissolving microneedles for controlled local administration of aconitine, Int. J. Pharm. 572 (2019) 118741. https://doi.org/10.1016/j.ijpharm.2019.118741.

[146] A.D. Permana, I.A. Tekko, M.T.C. McCrudden, Q.K. Anjani, D. Ramadon, H.O. McCarthy, R.F. Donnelly, Solid lipid nanoparticle-based dissolving microneedles: A promising intradermal lymph targeting drug delivery system with potential for enhanced treatment of lymphatic filariasis, J. Control. Release. 316 (2019) 34-52. https://doi.org/10.1016/j.jconrel.2019.10.004.

[147] S.G. Ingle, R. V Pai, J.D. Monpara, P.R. Vavia, Liposils: An effective strategy for stabilizing Paclitaxel loaded liposomes by surface coating with silica, Eur. J. Pharm. Sci. 122 (2018) 5163. https://doi.org/https://doi.org/10.1016/j.ejps.2018.06.025.

[148] Á. Cárcamo-Martínez, B. Mallon, J. Domínguez-Robles, A.S. Cordeiro, M. Celentano, E. Larrañeta, S.E.J. Bell, R.F. Donnelly, Plasmonic photothermal microneedle arrays and single needles for minimally-invasive deep in-skin hyperthermia., J. Mater. Chem. B. 8 (2020) 54255433. https://doi.org/10.1039/d0tb00962h.

[149] N. Rasenack, B.W. Müller, Micron-Size Drug Particles: Common and Novel Micronization Techniques, Pharm. Dev. Technol. 9 (2004) 1-13. https://doi.org/10.1081/PDT-120027417.

[150] G.G. Liversidge, K.C. Cundy, Particle size reduction for improvement of oral bioavailability of hydrophobic drugs: I. Absolute oral bioavailability of nanocrystalline danazol in beagle dogs, Int. J. Pharm. 125 (1995) 91-97. https://doi.org/10.1016/0378-5173(95)00122-Y.

[151] R.H. Müller, S. Gohla, C.M. Keck, State of the art of nanocrystals - Special features, production, nanotoxicology aspects and intracellular delivery, Eur. J. Pharm. Biopharm. 78 (2011) 1-9. https://doi.org/http://dx.doi.org/10.1016/j.ejpb.2011.01.007.

[152] A.J. Paredes, J.M. Llabot, S. Sánchez Bruni, D. Allemandi, S.D. Palma, Self-dispersible nanocrystals of albendazole produced by high pressure homogenization and spray-drying, Drug Dev. Ind. Pharm. 42 (2016) 1564-1570. https://doi.org/10.3109/03639045.2016.1151036.

[153] A. Paredes, N. Camacho, L. Schofs, A. Dib, M. Zarazaga, N. Litterio, D. Allemandi, S. Sánchez Bruni, C. Lanusse, S. Palma, Ricobendazole nanocrystals obtained by media milling and spray drying: pharmacokinetic comparison with the micronized form of the drug, Int. J. Pharm. 585 (2020) 119501. https://doi.org/10.1016/j.ijpharm.2020.119501.

[154] B.X. Camiletti, N.M. Camacho, A.J. Paredes, D.A. Allemandi, S.D. Palma, N. Grosso, Selfdispersible nanocrystals of azoxystrobin and cyproconazole with increased efficacy against soilborne fungal pathogens isolated from peanut crops, Powder Technol. 0 (2020) Article in press. https://doi.org/https://doi.org/10.1016/j.powtec.2020.06.025.

[155] M.E. Melian, A. Paredes, B. Munguía, M. Colobbio, J.C. Ramos, R. Teixeira, E. Manta, S. Palma, R. Faccio, L. Domínguez, Nanocrystals of Novel Valerolactam-Fenbendazole Hybrid with Improved in vitro Dissolution Performance, 21 (2020) 1-15. 
https://doi.org/10.1208/s12249-020-01777-y.

[156] B. Sun, Y. Yeo, Nanocrystals for the parenteral delivery of poorly water-soluble drugs, Curr. Opin. Solid State Mater. Sci. 16 (2012) 295-301. https://doi.org/10.1016/j.cossms.2012.10.004.

[157] R. Mauludin, R.H. Müller, C.M. Keck, Development of an oral rutin nanocrystal formulation, Int. J. Pharm. (2009). https://doi.org/10.1016/j.ijpharm.2008.11.029.

[158] A.J. Paredes, N. Litterio, A. Dib, D.A. Allemandi, C. Lanusse, S.S. Bruni, S.D. Palma, A nanocrystal-based formulation improves the pharmacokinetic performance and therapeutic response of albendazole in dogs, J. Pharm. Pharmacol. 70 (2018). https://doi.org/10.1111/jphp.12834.

[159] P. Pensel, A. Paredes, C.M. Albani, D. Allemandi, S. Sanchez Bruni, S.D. Palma, M.C. Elissondo, Albendazole nanocrystals in experimental alveolar echinococcosis: Enhanced chemoprophylactic and clinical efficacy in infected mice, Vet. Parasitol. 251 (2018) 78-84. https://doi.org/10.1016/j.vetpar.2017.12.022.

[160] S. Abdelghany, I.A. Tekko, L. Vora, E. Larrañeta, A.D. Permana, R.F. Donnelly, Nanosuspension-based dissolving microneedle arrays for intradermal delivery of curcumin, Pharmaceutics. 11 (2019) 308. https://doi.org/10.3390/pharmaceutics11070308.

[161] A.D. Permana, M.T.C. McCrudden, R.F. Donnelly, Enhanced intradermal delivery of nanosuspensions of antifilariasis drugs using dissolving microneedles: A proof of concept study, Pharmaceutics. 11 (2019) 346. https://doi.org/10.3390/pharmaceutics11070346.

[162] M. He, G. Yang, S. Zhang, X. Zhao, Y. Gao, Dissolving Microneedles Loaded With Etonogestrel Microcrystal Particles for Intradermal Sustained Delivery, J. Pharm. Sci. 107 (2018) 1037-1045. https://doi.org/10.1016/j.xphs.2017.11.013.

[163] M.T.C. Mc Crudden, E. Larrañeta, A. Clark, C. Jarrahian, A. Rein-Weston, S. Lachau-Durand, N. Niemeijer, P. Williams, C. Haeck, H.O. McCarthy, D. Zehrung, R.F. Donnelly, Design, formulation and evaluation of novel dissolving microarray patches containing a long-acting rilpivirine nanosuspension, J. Control. Release. $28 \quad$ (2018) 119-129. https://doi.org/10.1016/j.jconrel.2018.11.002.

[164] M.T.C. McCrudden, E. Larrañeta, A. Clark, C. Jarrahian, A. Rein-Weston, B. Creelman, Y. Moyo, S. Lachau-Durand, N. Niemeijer, P. Williams, H.O. McCarthy, D. Zehrung, R.F. Donnelly, Design, formulation, and evaluation of novel dissolving microarray patches containing rilpivirine for intravaginal delivery, Adv. Healthc. Mater. 8 (2019) 1801510. https://doi.org/10.1002/adhm.201801510.

[165] A.J. Paredes, P.E. Mckenna, I.K. Ramöller, Y.A. Naser, F. Volpe-zanutto, M. Li, M.T.A. Abbate, L. Zhao, C. Zhang, J.M. Abu-ershaid, X. Dai, R.F. Donnelly, Microarray Patches: Poking a Hole in the Challenges Faced When Delivering Poorly Soluble Drugs, Adv. Funct. Mater. 2005792 (2020) 1-27. https://doi.org/10.1002/adfm.202005792.

[166] P.S. Giffen, R. Bhuiya, K. Brackenborough, M.J. Hobbs, L. Qian, M.D. Burke, Controlled Delivery of Dutasteride Using Dissolvable Microarrays: Initial Formulation and In Vivo Evaluation, J. Pharm. Sci. 109 (2020) 1303-1311. https://doi.org/10.1016/j.xphs.2019.11.012.

[167] R.F. Donnelly, E. Larrañeta, Microarray patches: potentially useful delivery systems for longacting nanosuspensions, Drug Discov. Today. 23 (2018) 1026-1033. https://doi.org/10.1016/j.drudis.2017.10.013.

[168] M.Y. Kim, B. Jung, J.H. Park, Hydrogel swelling as a trigger to release biodegradable polymer microneedles in skin, Biomaterials. 33 (2012) 668-678. https://doi.org/10.1016/j.biomaterials.2011.09.074.

[169] X. Hong, L. Wei, F. Wu, Z. Wu, L. Chen, Z. Liu, W. Yuan, Dissolving and biodegradable microneedle technologies for transdermal sustained delivery of drug and vaccine, Drug Des. Devel. Ther. 7 (2013) 945-952. https://doi.org/10.2147/DDDT.S44401.

[170] H.K. Makadia, S.J. Siegel, Poly Lactic-co-Glycolic Acid (PLGA) as biodegradable controlled drug delivery carrier, Polymers (Basel). 3 (2011) 1377-1397. https://doi.org/10.3390/polym3031377.

[171] L. Vora, S. V G, P. Vavia, Zero order controlled release delivery of cholecalciferol from injectable biodegradable microsphere: In-vitro characterization and in-vivo pharmacokinetic studies, Eur. J. Pharm. Sci. 107 (2017) 78-86. https://doi.org/10.1016/j.ejps.2017.06.027.

[172] S. Fredenberg, M. Wahlgren, M. Reslow, A. Axelsson, The mechanisms of drug release in 
poly(lactic-co-glycolic acid)-based drug delivery systems - A review, Int. J. Pharm. 415 (2011) 34-52. https://doi.org/10.1016/j.ijpharm.2011.05.049.

[173] P.C. Demuth, J.J. Moon, H. Suh, P.T. Hammond, D.J. Irvine, Releasable layer-by-layer assembly of stabilized lipid nanocapsules on microneedles for enhanced transcutaneous vaccine delivery, ACS Nano. 6 (2012) 8041-8051. https://doi.org/10.1021/nn302639r.

[174] A. Than, K. Liang, S. Xu, L. Sun, H. Duan, F. Xi, C. Xu, P. Chen, Transdermal delivery of antiobesity compounds to subcutaneous adipose tissue with polymeric microneedle patches, Small Methods. 1 (2017) 1700269. https://doi.org/10.1002/smtd.201700269.

[175] K.J. Lee, H.B. Song, W. Cho, J.H. Kim, J.H. Kim, W.H. Ryu, Intracorneal injection of a detachable hybrid microneedle for sustained drug delivery, Acta Biomater. 80 (2018) 48-57. https://doi.org/10.1016/j.actbio.2018.09.039.

[176] S.C. Park, M.J. Kim, S.K. Baek, J.H. Park, S.O. Choi, Spray-formed layered polymer microneedles for controlled biphasic drug delivery, Polymers (Basel). 11 (2019) 369. https://doi.org/10.3390/POLYM11020369.

[177] K.J. Lee, Y. Xue, J. Lee, H.J. Kim, Y. Liu, P. Tebon, E. Sarikhani, W. Sun, S. Zhang, R. Haghniaz, B. Çelebi-Saltik, X. Zhou, S. Ostrovidov, S. Ahadian, N. Ashammakhi, M.R. Dokmeci, A. Khademhosseini, A Patch of Detachable Hybrid Microneedle Depot for Localized Delivery of Mesenchymal Stem Cells in Regeneration Therapy, Adv. Funct. Mater. 30 (2020) 2000086. https://doi.org/10.1002/adfm.202000086.

[178] N.K. Palakurthi, Z.M. Correa, J.J. Augsburger, R.K. Banerjee, Toxicity of a biodegradable microneedle implant loaded with methotrexate as a sustained release device in normal rabbit eye: A pilot study, J. Ocul. Pharmacol. Ther. 27 (2011) 151-156. https://doi.org/10.1089/jop.2010.0037.

[179] M.A. Woodruff, D.W. Hutmacher, The return of a forgotten polymer - Polycaprolactone in the 21st century, Prog. Polym. Sci. $35 \quad$ (2010) 1217-1256. https://doi.org/10.1016/j.progpolymsci.2010.04.002.

[180] E. Rudnik, Compostable Polymer Materials, Elsevier Ltd, 2008. https://doi.org/10.1016/B9780-08-045371-2.X5001-X.

[181] T.E. Andersen, A.J. Andersen, R.S. Petersen, L.H. Nielsen, S.S. Keller, Drug loaded biodegradable polymer microneedles fabricated by hot embossing, Microelectron. Eng. 195 (2018) 57-61. https://doi.org/10.1016/j.mee.2018.03.024.

[182] M.C. Chen, K.W. Wang, D.H. Chen, M.H. Ling, C.Y. Liu, Remotely triggered release of small molecules from LaB6@SiO2-loaded polycaprolactone microneedles, Acta Biomater. 13 (2015) 344-353. https://doi.org/10.1016/j.actbio.2014.11.040.

[183] Y. Zhang, D. Chai, M. Gao, B. Xu, G. Jiang, Thermal ablation of separable microneedles for transdermal delivery of metformin on diabetic rats, Int. J. Polym. Mater. Polym. Biomater. 68 (2019) 850-858. https://doi.org/10.1080/00914037.2018.1517347.

[184] M.C. Chen, Z.W. Lin, M.H. Ling, Near-infrared light-activatable microneedle system for treating superficial tumors by combination of chemotherapy and photothermal therapy, ACS Nano. 10 (2016) 93-101. https://doi.org/10.1021/acsnano.5b05043.

[185] M.C. Chen, H.A. Chan, M.H. Ling, L.C. Su, Implantable polymeric microneedles with phototriggerable properties as a patient-controlled transdermal analgesia system, J. Mater. Chem. B. 5 (2017) 496-503. https://doi.org/10.1039/c6tb02718k.

[186] M.C. Chen, M.H. Ling, K.W. Wang, Z.W. Lin, B.H. Lai, D.H. Chen, Near-infrared lightresponsive composite microneedles for on-demand transdermal drug delivery, Biomacromolecules. 16 (2015) 1598-1607. https://doi.org/10.1021/acs.biomac.5b00185.

[187] W. Yu, G. Jiang, Y. Zhang, D. Liu, B. Xu, J. Zhou, Near-infrared light triggered and separable microneedles for transdermal delivery of metformin in diabetic rats, J. Mater. Chem. B. 5 (2017) 9507-9513. https://doi.org/10.1039/c7tb02236k.

[188] J. Cheng, G. Chen, Y. Zhu, Fe3O4/Polycaprolactone Microneedles with Controlled Drug Delivery and Magnetic Hyperthermia, Nano Adv. 2 (2017) 29-35. https://doi.org/10.22180/na200.

[189] G.H. Altman, F. Diaz, C. Jakuba, T. Calabro, R.L. Horan, J. Chen, H. Lu, J. Richmond, D.L. Kaplan, Silk-based biomaterials, Biomaterials. 24 (2003) 401-416. https://doi.org/https://doi.org/10.1016/S0142-9612(02)00353-8. 
[190] C. Jiang, X. Wang, R. Gunawidjaja, Y.-H. Lin, M.K. Gupta, D.L. Kaplan, R.R. Naik, V.V. Tsukruk, Mechanical Properties of Robust Ultrathin Silk Fibroin Films, Adv. Funct. Mater. 17 (2007) 2229-2237. https://doi.org/10.1002/adfm.200601136.

[191] W. Huang, S. Ling, C. Li, F.G. Omenetto, D.L. Kaplan, Silkworm silk-based materials and devices generated using bio-nanotechnology, Chem. Soc. Rev. 47 (2018) 6486-6504. https://doi.org/10.1039/c8cs00187a.

[192] A.B. Li, J.A. Kluge, N.A. Guziewicz, F.G. Omenetto, D.L. Kaplan, Silk-based stabilization of biomacromolecules, J. Control. Release. $219 \quad$ (2015) 416-430. https://doi.org/10.1016/j.jconrel.2015.09.037.

[193] Z. Yin, D. Kuang, S. Wang, Z. Zheng, V.K. Yadavalli, S. Lu, Swellable silk fibroin microneedles for transdermal drug delivery, Int. J. Biol. Macromol. 106 (2018) 48-56. https://doi.org/https://doi.org/10.1016/j.ijbiomac.2017.07.178.

[194] N. Mali, P. Wavikar, P. Vavia, Serratiopeptidase loaded chitosan nanoparticles by polyelectrolyte complexation: in vitro and in vivo evaluation, AAPS PharmSciTech. 16 (2015) 59-66. https://doi.org/10.1208/s12249-014-0201-0.

[195] S. Bhatnagar, P.R. Gadeela, P. Thathireddy, V.V.K. Venuganti, Microneedle-based drug delivery: materials of construction, J. Chem. Sci. 131 (2019). https://doi.org/10.1007/s12039019-1666-x.

[196] D. Fong, C.D. Hoemann, Chitosan immunomodulatory properties: perspectives on the impact of structural properties and dosage, Futur. Sci. OA. 4 (2017) FSO225-FSO225. https://doi.org/10.4155/fsoa-2017-0064.

[197] Y. Xie, B. Xu, Y. Gao, Controlled transdermal delivery of model drug compounds by MEMS microneedle array, Nanomedicine Nanotechnology, Biol. Med. 1 (2005) 184-190. https://doi.org/10.1016/j.nano.2005.03.001.

[198] A. Marin, A.K. Andrianov, Carboxymethylcellulose-Chitosan-coated microneedles with modulated hydration properties, J. Appl. Polym. Sci. 121 (2011) 391-405. https://doi.org/10.1002/app.33608.

[199] Z. Hu, C.S. Meduri, R.S.J. Ingrole, H.S. Gill, G. Kumar, Solid and hollow metallic glass microneedles for transdermal drug-delivery, Appl. Phys. Lett. 116 (2020) 203703. https://doi.org/10.1063/5.0008983.

[200] J.H. Park, M.G. Allen, M.R. Prausnitz, Biodegradable polymer microneedles: Fabrication, mechanics and transdermal drug delivery, J. Control. Release. 104 (2005) 51-66. https://doi.org/10.1016/j.jconrel.2005.02.002.

[201] Y.C. Kim, J.H. Park, M.R. Prausnitz, Microneedles for drug and vaccine delivery, Adv. Drug Deliv. Rev. 64 (2012) 1547-1568. https://doi.org/10.1016/j.addr.2012.04.005.

[202] R. Paleco, S.R. Vučen, A.M. Crean, A. Moore, S. Scalia, Enhancement of the in vitro penetration of quercetin through pig skin by combined microneedles and lipid microparticles, Int. J. Pharm. 472 (2014) 206-213. https://doi.org/10.1016/j.ijpharm.2014.06.010.

[203] A. Nayak, D.B. Das, G.T. Vladisavljević, Microneedle-assisted permeation of lidocaine carboxymethylcellulose with gelatine Co-polymer hydrogel, Pharm. Res. 31 (2014) 1170-1184. https://doi.org/10.1007/s1 1095-013-1240-z.

[204] L.M. Bonati, G.K. Epstein, T.L. Strugar, Microneedling in all skin types: A review, J. Drugs Dermatology. 16 (2017) 308-314.

[205] Y. Guo, Xindong; Yang, Conductive polymer microneedle paster for controllable drug release and preparation method thereof, CN 110201296, 2019.

[206] D.G. Koutsonanos, E. V Vassilieva, A. Stavropoulou, V.G. Zarnitsyn, E.S. Esser, M.T. Taherbhai, M.R. Prausnitz, R.W. Compans, I. Skountzou, skin with microneedles improves protection, (2012) 1-10. https://doi.org/10.1038/srep00357.

[207] Y. Hao, W. Li, X.L. Zhou, F. Yang, Z.Y. Qian, Microneedles-based transdermal drug delivery systems: A review, J. Biomed. Nanotechnol. $13 \quad$ (2017) 1581-1597. https://doi.org/10.1166/jbn.2017.2474.

[208] Y. Zhang, K. Brown, K. Siebenaler, A. Determan, D. Dohmeier, K. Hansen, Development of lidocaine-coated microneedle product for rapid, safe, and prolonged local analgesic action, Pharm. Res. 29 (2012) 170-177. https://doi.org/10.1007/s11095-011-0524-4.

[209] S.H. van Rijt, D.A. Bölükbas, C. Argyo, S. Datz, M. Lindner, O. Eickelberg, M. Königshoff, T. 
Bein, S. Meiners, Protease-mediated release of chemotherapeutics from mesoporous silica nanoparticles to ex vivo human and mouse lung tumors., ACS Nano. 9 (2015) 2377-2389. https://doi.org/10.1021/nn5070343.

[210] D.A. Davis, A. Hamilton, J. Yang, L.D. Cremar, D. Van Gough, S.L. Potisek, M.T. Ong, P. V Braun, T.J. Martínez, S.R. White, J.S. Moore, N.R. Sottos, Force-induced activation of covalent bonds in mechanoresponsive polymeric materials., Nature. 459 (2009) 68-72. https://doi.org/10.1038/nature07970.

[211] S. Dai, P. Ravi, K.C. Tam, pH-Responsive polymers: synthesis $\{$,$\} properties and applications,$ Soft Matter. 4 (2008) 435-449. https://doi.org/10.1039/B714741D.

[212] T. Tanaka, I. Nishio, S.T. Sun, S. Ueno-Nishio, Collapse of gels in an electric field., Science. 218 (1982) 467-469. https://doi.org/10.1126/science.218.4571.467.

[213] J. Thévenot, H. Oliveira, O. Sandre, S. Lecommandoux, Magnetic responsive polymer composite materials, Chem. Soc. Rev. 42 (2013) 7099-7116. https://doi.org/10.1039/C3CS60058K.

[214] W. Park, B. Bae, K. Na, A highly tumor-specific light-triggerable drug carrier responds to hypoxic tumor conditions for effective tumor treatment., Biomaterials. 77 (2016) 227-234. https://doi.org/10.1016/j.biomaterials.2015.11.014.

[215] Á. Cárcamo-Martínez, J. Domínguez-Robles, B. Mallon, M.T. Raman, A.S. Cordeiro, S.E.J. Bell, E. Larrañeta, R.F. Donnelly, Potential of polymeric films loaded with gold nanorods for local hyperthermia applications, Nanomaterials. $10 \quad$ (2020) 1-17. https://doi.org/10.3390/nano10030582.

[216] Y. Zhao, Y. Guo, L. Tang, Engineering cancer vaccines using stimuli-responsive biomaterials, Nano Res. 11 (2018) 5355-5371.

[217] J.G. Hardy, E. Larrañeta, R.F. Donnelly, N. McGoldrick, K. Migalska, M.T.C. McCrudden, N.J. Irwin, L. Donnelly, C.P. McCoy, Hydrogel-Forming Microneedle Arrays Made from LightResponsive Materials for On-Demand Transdermal Drug Delivery, Mol. Pharm. 13 (2016) 907914.

[218] D. Liu, Y. Zhang, G. Jiang, W. Yu, B. Xu, J. Zhu, Fabrication of Dissolving Microneedles with Thermal-Responsive Coating for NIR-Triggered Transdermal Delivery of Metformin on Diabetic Rats, ACS Biomater. Sci. Eng. 4 (2018) 1687-1695. https://doi.org/10.1021/acsbiomaterials.8b00159.

[219] J. Yu, Y. Zhang, H. Bomba, Z. Gu, Stimuli-Responsive Delivery of Therapeutics for Diabetes Treatment., Bioeng. Transl. Med. 1 (2016) 323-337. https://doi.org/10.1002/btm2.10036.

[220] A.M. de Groot, G. Du, J. Mönkäre, A.C.M. Platteel, F. Broere, J.A. Bouwstra, A.J.A.M. Sijts, Hollow microneedle-mediated intradermal delivery of model vaccine antigen-loaded PLGA nanoparticles elicits protective $\mathrm{T}$ cell-mediated immunity to an intracellular bacterium, J. Control. $\quad$ Release. $266 \quad$ (2017) 27-35. https://doi.org/https://doi.org/10.1016/j.jconrel.2017.09.017.

[221] P.M. Wang, M. Cornwell, J. Hill, M.R. Prausnitz, Precise Microinjection into Skin Using Hollow Microneedles, J. Invest. Dermatol. $126 \quad$ (2006) 1080-1087. https://doi.org/https://doi.org/10.1038/sj.jid.5700150.

[222] U.O. Häfeli, A. Mokhtari, D. Liepmann, B. Stoeber, In vivo evaluation of a microneedle-based miniature syringe for intradermal drug delivery., Biomed. Microdevices. 11 (2009) 943-950. https://doi.org/10.1007/s10544-009-9311-y.

[223] G. Du, R.M. Hathout, M. Nasr, M.R. Nejadnik, J. Tu, R.I. Koning, A.J. Koster, B. Slütter, A. Kros, W. Jiskoot, J.A. Bouwstra, J. Mönkäre, Intradermal vaccination with hollow microneedles: A comparative study of various protein antigen and adjuvant encapsulated nanoparticles, J. Control. Release. $266 \quad$ (2017) 109-118. https://doi.org/10.1016/j.jconrel.2017.09.021.

[224] J. Jiang, J.S. Moore, H.F. Edelhauser, M.R. Prausnitz, Intrascleral drug delivery to the eye using hollow microneedles., Pharm. Res. 26 (2009) 395-403. https://doi.org/10.1007/s11095-0089756-3.

[225] S.R. Patel, A.S.P. Lin, H.F. Edelhauser, M.R. Prausnitz, Suprachoroidal drug delivery to the back of the eye using hollow microneedles, Pharm. Res. 28 (2011) 166-176. https://doi.org/10.1007/s11095-010-0271-y. 
[226] Y.C. Kim, H.F. Edelhauser, M.R. Prausnitz, Particle-stabilized emulsion droplets for gravitymediated targeting in the posterior segment of the eye, Adv. Healthc. Mater. 3 (2014) 12721282. https://doi.org/10.1002/adhm.201300696.

[227] J. Shen, D.J. Burgess, Long Acting Injections and Implants, Long Act. Inject. Implant. (2012) 73-91. https://doi.org/10.1007/978-1-4614-0554-2.

[228] J.-F. Jin, L.-L. Zhu, M. Chen, H.-M. Xu, H.-F. Wang, X.-Q. Feng, X.-P. Zhu, Q. Zhou, The optimal choice of medication administration route regarding intravenous, intramuscular, and subcutaneous injection., Patient Prefer. Adherence. 9 (2015) 923-942. https://doi.org/10.2147/PPA.S87271.

[229] A.D. AlQahtani, D. O'Connor, A. Domling, S.K. Goda, Strategies for the production of longacting therapeutics and efficient drug delivery for cancer treatment, Biomed. Pharmacother. 113 (2019) 108750. https://doi.org/https://doi.org/10.1016/j.biopha.2019.108750.

[230] C.L. Stevenson, C.A. Rhodes, S.J. Prestrelski, Delivery of Peptides and Proteins via Long Acting Injections and Implants BT - Long Acting Injections and Implants, in: J.C. Wright, D.J. Burgess (Eds.), Springer US, Boston, MA, 2012: pp. 409-427. https://doi.org/10.1007/978-14614-0554-2_20.

[231] Y. Kapoor, M. Milewski, L. Dick, J. Zhang, J.R. Bothe, M. Gehrt, K. Manser, B. Nissley, I. Petrescu, P. Johnson, S. Burton, J. Moseman, V. Hua, T. Grunewald, M. Tomai, R. Smith, Coated microneedles for transdermal delivery of a potent pharmaceutical peptide, Biomed. Microdevices. 22 (2019) 7. https://doi.org/10.1007/s10544-019-0462-1.

[232] J.K. Hickling, K.R. Jones, M. Friede, D. Zehrung, D. Chen, D. Kristensenc, Intradermal delivery of vaccines: Potential benefits and current challenges, Bull. World Health Organ. 89 (2011) 221226. https://doi.org/10.2471/BLT.10.079426.

[233] J.C. Joyce, H.E. Sella, H. Jost, M.J. Mistilis, E.S. Esser, P. Pradhan, R. Toy, M.L. Collins, P.A. Rota, K. Roy, I. Skountzou, R.W. Compans, M.S. Oberste, W.C. Weldon, J.J. Norman, M.R. Prausnitz, Extended delivery of vaccines to the skin improves immune responses, J. Control. Release. 304 (2019) 135-145. https://doi.org/https://doi.org/10.1016/j.jconrel.2019.05.006.

[234] Y.C. Kim, C. Jarrahian, D. Zehrung, S. Mitragotri, M.R. Prausnitz, Delivery systems for intradermal vaccination., Curr. Top. Microbiol. Immunol. 351 (2012) 77-112. https://doi.org/10.1007/82_2011_123.

[235] J.J. Norman, J. Gupta, S.R. Patel, S. Park, C. Jarrahian, D. Zehrung, M.R. Prausnitz, Reliability and accuracy of intradermal injection by Mantoux technique, hypodermic needle adapter, and hollow microneedle in pigs, Drug Deliv. Transl. Res. 4 (2014) 126-130. https://doi.org/10.1007/s13346-013-0184-5.

[236] M.R. Prausnitz, Engineering Microneedle Patches for Vaccination and Drug Delivery to Skin, Annu. Rev. Chem. Biomol. Eng. 8 (2017) 177-200. https://doi.org/10.1146/annurevchembioeng-060816-101514.

[237] S. Marshall, L.J. Sahm, A.C. Moore, The success of microneedle-mediated vaccine delivery into skin, Hum. Vaccin. Immunother. 12 (2016) 2975-2983. https://doi.org/10.1080/21645515.2016.1171440.

[238] A.M. Rodgers, A.S. Cordeiro, A. Kissenpfennig, R.F. Donnelly, Microneedle arrays for vaccine delivery: the possibilities, challenges and use of nanoparticles as a combinatorial approach for enhanced vaccine immunogenicity, Expert Opin. Drug Deliv. 15 (2018) 851-867. https://doi.org/10.1080/17425247.2018.1505860.

[239] J. Arya, S. Henry, H. Kalluri, D. V McAllister, W.P. Pewin, M.R. Prausnitz, Tolerability, usability and acceptability of dissolving microneedle patch administration in human subjects., Biomaterials. 128 (2017) 1-7. https://doi.org/10.1016/j.biomaterials.2017.02.040.

[240] G.J.P. Fernando, J. Hickling, C.M. Jayashi Flores, P. Griffin, C.D. Anderson, S.R. Skinner, C. Davies, K. Witham, M. Pryor, J. Bodle, S. Rockman, I.H. Frazer, A.H. Forster, Safety, tolerability, acceptability and immunogenicity of an influenza vaccine delivered to human skin by a novel high-density microprojection array patch (Nanopatch ${ }^{\mathrm{TM}}$ )., Vaccine. 36 (2018) 37793788. https://doi.org/10.1016/j.vaccine.2018.05.053.

[241] N.G. Rouphael, M. Paine, R. Mosley, S. Henry, D. V McAllister, H. Kalluri, W. Pewin, P.M. Frew, T. Yu, N.J. Thornburg, S. Kabbani, L. Lai, E. V Vassilieva, I. Skountzou, R.W. Compans, M.J. Mulligan, M.R. Prausnitz, The safety, immunogenicity, and acceptability of inactivated 
influenza vaccine delivered by microneedle patch (TIV-MNP 2015): a randomised, partly blinded, placebo-controlled, phase 1 trial., Lancet (London, England). 390 (2017) 649-658. https://doi.org/10.1016/S0140-6736(17)30575-5.

[242] A. V Boopathy, A. Mandal, D.W. Kulp, S. Menis, N.R. Bennett, H.C. Watkins, W. Wang, J.T. Martin, N.T. Thai, Y. He, W.R. Schief, P.T. Hammond, D.J. Irvine, Enhancing humoral immunity via sustained-release implantable microneedle patch vaccination, Proc. Natl. Acad. Sci. 116 (2019) 16473-16478. https://doi.org/10.1073/pnas.1902179116.

[243] J.D. Monpara, S.J. Sodha, P.K. Gupta, COVID-19 associated complications and potential $\begin{array}{lllllll}\text { therapeutic targets, } & \text { Eur. J. } & \text { Pharmacol. } 886 & \text { (2020) }\end{array}$ https://doi.org/https://doi.org/10.1016/j.ejphar.2020.173548.

[244] J. Machhi, J. Herskovitz, A.M. Senan, D. Dutta, B. Nath, M.D. Oleynikov, W.R. Blomberg, D.D. Meigs, M. Hasan, M. Patel, P. Kline, R.C.C. Chang, L. Chang, H.E. Gendelman, B.D. Kevadiya, The Natural History, Pathobiology, and Clinical Manifestations of SARS-CoV-2 Infections, J. Neuroimmune Pharmacol. (2020). https://doi.org/10.1007/s11481-020-09944-5.

[245] E. Kim, G. Erdos, S. Huang, T.W. Kenniston, S.C. Balmert, C.D. Carey, V.S. Raj, M.W. Epperly, W.B. Klimstra, B.L. Haagmans, E. Korkmaz, L.D. Falo Jr., A. Gambotto, Microneedle array delivered recombinant coronavirus vaccines: Immunogenicity and rapid translational development, EBioMedicine. 55 (2020). https://doi.org/10.1016/j.ebiom.2020.102743.

[246] F.-S. Quan, Y.-C. Kim, A. Vunnava, D.-G. Yoo, J.-M. Song, M.R. Prausnitz, R.W. Compans, S.-M. Kang, Intradermal Vaccination with Influenza Virus-Like Particles by Using Microneedles Induces Protection Superior to That with Intramuscular Immunization, J. Virol. 84 (2010) 7760-7769. https://doi.org/10.1128/JVI.01849-09.

[247] H.J. Corbett, G.J.P. Fernando, X. Chen, I.H. Frazer, M.A.F. Kendall, Skin vaccination against cervical cancer associated human papillomavirus with a novel micro-projection array in a mouse model., PLoS One. 5 (2010) e13460. https://doi.org/10.1371/journal.pone.0013460.

[248] E. Gorell, N. Nguyen, A. Lane, Z. Siprashvili, Gene therapy for skin diseases, Cold Spring Harb. Perspect. Med. 4 (2014) a015149-a015149. https://doi.org/10.1101/cshperspect.a015149.

[249] L. Vora, M. Tyagi, K. Patel, S. Gupta, P. Vavia, Self-assembled nanocomplexes of anionic pullulan and polyallylamine for DNA and $\mathrm{pH}$-sensitive intracellular drug delivery, J. Nanoparticle Res. (2014). https://doi.org/10.1007/s11051-014-2781-8.

[250] J. McCaffrey, R.F. Donnelly, H.O. McCarthy, Microneedles: an innovative platform for gene delivery., Drug Deliv. Transl. Res. 5 (2015) 424-437. https://doi.org/10.1007/s13346-015-02431.

[251] M. Qu, H.J. Kim, X. Zhou, C. Wang, X. Jiang, J. Zhu, Y. Xue, P. Tebon, S.A. Sarabi, S. Ahadian, M.R. Dokmeci, S. Zhu, Z. Gu, W. Sun, A. Khademhosseini, Biodegradable microneedle patch for transdermal gene delivery, Nanoscale. 12 (2020) 16724-16729. https://doi.org/10.1039/d0nr02759f.

[252] A. Makkouk, G.J. Weiner, Cancer immunotherapy and breaking immune tolerance: new approaches to an old challenge., Cancer Res. 75 (2015) 5-10. https://doi.org/10.1158/00085472.CAN-14-2538.

[253] W. Chen, H. Li, D. Shi, Z. Liu, W. Yuan, Microneedles As a Delivery System for Gene Therapy, Front. Pharmacol. 7 (2016) 137. https://doi.org/10.3389/fphar.2016.00137.

[254] M. Zaric, O. Lyubomska, O. Touzelet, C. Poux, S. Al-Zahrani, F. Fay, L. Wallace, D. Terhorst, B. Malissen, S. Henri, U.F. Power, C.J. Scott, R.F. Donnelly, A. Kissenpfennig, Skin dendritic cell targeting via microneedle arrays laden with antigen-encapsulated poly- D, 1 -Lactide- Co Glycolide nanoparticles induces efficient antitumor and antiviral immune responses, ACS Nano. 7 (2013) 2042-2055. https://doi.org/10.1021/nn304235j.

[255] M. Wang, Y. Han, X. Yu, L. Liang, H. Chang, D.C. Yeo, C. Wiraja, M.L. Wee, L. Liu, X. Liu, C. Xu, Upconversion Nanoparticle Powered Microneedle Patches for Transdermal Delivery of siRNA, Adv. Healthc. Mater. 9 (2020) 1900635. https://doi.org/10.1002/adhm.201900635.

[256] J. Birchall, S. Coulman, M. Pearton, C. Allender, K. Brain, A. Anstey, C. Gateley, N. Wilke, A. Morrissey, Cutaneous DNA delivery and gene expression in ex vivo human skin explants via wet-etch microfabricated microneedles, J. Drug Target. 13 (2005) 415-421. https://doi.org/10.1080/10611860500383705.

[257] H.T.T. Duong, Y. Yin, T. Thambi, T.L. Nguyen, V.H. Giang Phan, M.S. Lee, J.E. Lee, J. Kim, 
J.H. Jeong, D.S. Lee, Smart vaccine delivery based on microneedle arrays decorated with ultrapH-responsive copolymers for cancer immunotherapy., Biomaterials. 185 (2018) 13-24. https://doi.org/10.1016/j.biomaterials.2018.09.008.

[258] X. Ke, G.P. Howard, H. Tang, B. Cheng, M.T. Saung, J.L. Santos, H.-Q. Mao, Physical and chemical profiles of nanoparticles for lymphatic targeting, Adv. Drug Deliv. Rev. 151-152 (2019) 72-93. https://doi.org/https://doi.org/10.1016/j.addr.2019.09.005.

[259] F.O. Nestle, P. Di Meglio, J.-Z. Qin, B.J. Nickoloff, Skin immune sentinels in health and disease., Nat. Rev. Immunol. 9 (2009) 679-691. https://doi.org/10.1038/nri2622.

[260] L. Niu, L.Y. Chu, S.A. Burton, K.J. Hansen, J. Panyam, Intradermal delivery of vaccine nanoparticles using hollow microneedle array generates enhanced and balanced immune $\begin{array}{llllll}\text { response, J. } & \text { Control. } & \text { Release. } & 294 & \text { (2019) }\end{array}$ https://doi.org/https://doi.org/10.1016/j.jconrel.2018.12.026.

[261] H.M. Patel, B. Katherine M., R. Vaughan-Jones, Assessment of the potential uses of liposomes for lymphoscintigraphy and lymphatic drug delivery failure of $99 \mathrm{~m}$-technetium marker to represent intact liposomes in lymph nodes, Biochim. Biophys. Acta - Gen. Subj. 801 (1984) 7686. https://doi.org/https://doi.org/10.1016/0304-4165(84)90214-9.

[262] C. Oussoren, J. Zuidema, D.J. Crommelin, G. Storm, Lymphatic uptake and biodistribution of liposomes after subcutaneous injection. II. Influence of liposomal size, lipid compostion and lipid dose., Biochim. Biophys. Acta. 1328 (1997) 261-272. https://doi.org/10.1016/s00052736(97)00122-3.

[263] R.F. Uren, R. Howman-Giles, J.F. Thompson, Patterns of lymphatic drainage from the skin in patients with melanoma., J. Nucl. Med. 44 (2003) 570-582.

[264] Y. Nishioka, H. Yoshino, Lymphatic targeting with nanoparticulate system, Adv. Drug Deliv. Rev. 47 (2001) 55-64. https://doi.org/https://doi.org/10.1016/S0169-409X(00)00121-6.

[265] K. Moffatt, C. Quinn, P.J. McCague, R.F. Donnelly, Exploration into the opinions of patients with HIV, healthcare professionals and the lay public of the use of microneedles in clinical practice: highlighting the translational potential for their role in HIV infection, Drug Deliv. Transl. Res. (2020). https://doi.org/10.1007/s13346-020-00848-8.

[266] A. Rein-Weston, I. Tekko, L. Vora, C. Jarrahian, B. Spreen, T. Scott, R. Donnelly, D. Zehrung, LB8. Microarray Patch Delivery of Long-Acting HIV PrEP and Contraception, Open Forum Infect. Dis. 6 (2019) S996-S996. https://doi.org/10.1093/ofid/ofz415.2491.

[267] V. Halpern, R.M. Stalter, D.H. Owen, L.J. Dorflinger, A. Lendvay, K.H. Rademacher, Towards the development of a longer-acting injectable contraceptive: past research and current trends, Contraception. $92 \quad$ (2015) 3-9. https://doi.org/https://doi.org/10.1016/j.contraception.2015.02.014.

[268] R.F. Donnelly, E. Larraňeta, Slowly dissolving intradermal microneedles, Nat. Biomed. Eng. 3 (2019) 169-170. https://doi.org/10.1038/s41551-019-0369-4.

[269] M. He, G. Yang, S. Zhang, X. Zhao, Y. Gao, Dissolving Microneedles Loaded With Etonogestrel Microcrystal Particles for Intradermal Sustained Delivery, J. Pharm. Sci. 107 (2018) 1037-1045. https://doi.org/10.1016/j.xphs.2017.11.013.

[270] T.S. Stroup, N. Gray, Management of common adverse effects of antipsychotic medications., World Psychiatry. 17 (2018) 341-356. https://doi.org/10.1002/wps.20567.

[271] K.W. Ng, Penetration Enhancement of Topical Formulations., Pharmaceutics. 10 (2018). https://doi.org/10.3390/pharmaceutics10020051.

[272] E.L.H. Spierings, J.L. Brandes, D.B. Kudrow, J. Weintraub, P.C. Schmidt, D.J. Kellerman, S.J. Tepper, Randomized, double-blind, placebo-controlled, parallel-group, multi-center study of the safety and efficacy of ADAM zolmitriptan for the acute treatment of migraine, Cephalalgia. 38 (2018) 215-224. https://doi.org/10.1177/0333102417737765.

[273] B. Dubois, H.H. Feldman, C. Jacova, J.L. Cummings, S.T. Dekosky, P. Barberger-Gateau, A. Delacourte, G. Frisoni, N.C. Fox, D. Galasko, S. Gauthier, H. Hampel, G.A. Jicha, K. Meguro, J. O'Brien, F. Pasquier, P. Robert, M. Rossor, S. Salloway, M. Sarazin, L.C. de Souza, Y. Stern, P.J. Visser, P. Scheltens, Revising the definition of Alzheimer's disease: a new lexicon., Lancet. Neurol. 9 (2010) 1118-1127. https://doi.org/10.1016/S1474-4422(10)70223-4.

[274] B. Winblad, J.C. Machado, Use of rivastigmine transdermal patch in the treatment of Alzheimer's disease., Expert Opin. Drug Deliv. 5 (2008) 1377-1386. 
https://doi.org/10.1517/17425240802542690.

[275] Phase 1 Pharmacokinetic (PK) Study to Evaluate Once-weekly Corplex Donepezil Transdermal Delivery System Compared to Daily Oral Administration of Aricept in Healthy Adult Subjects, (n.d.). $\quad$ https://www.nia.nih.gov/alzheimers/clinical-trials/donepezil-transdermal-patch (accessed June 29, 2020).

[276] M. Sadeghi, F. Ganji, S.M. Taghizadeh, B. Daraei, Preparation and Characterization of Rivastigmine Transdermal Patch Based on Chitosan Microparticles., Iran. J. Pharm. Res. IJPR. 15 (2016) 283-294.

[277] S.L. Rogers, L.T. Friedhoff, Pharmacokinetic and pharmacodynamic profile of donepezil $\mathrm{HCl}$ following single oral doses., Br. J. Clin. Pharmacol. 46 Suppl 1 (1998) 1-6. https://doi.org/10.1046/j.1365-2125.1998.0460s1001.x.

[278] M.C. Kearney, E. Caffarel-Salvador, S.J. Fallows, H.O. McCarthy, R.F. Donnelly, Microneedlemediated delivery of donepezil: Potential for improved treatment options in Alzheimer's disease, Eur. J. Pharm. Biopharm. 103 (2016) 43-50.

[279] J.Y. Kim, M.R. Han, Y.H. Kim, S.W. Shin, S.Y. Nam, J.H. Park, Tip-loaded dissolving microneedles for transdermal delivery of donepezil hydrochloride for treatment of Alzheimer's disease, Eur. J. Pharm. Biopharm. 105 (2016) 148-155. https://doi.org/10.1016/j.ejpb.2016.06.006.

[280] Y. Ye, J. Yu, C. Wang, N.-Y. Nguyen, G.M. Walker, J.B. Buse, Z. Gu, Microneedles Integrated with Pancreatic Cells and Synthetic Glucose-Signal Amplifiers for Smart Insulin Delivery., Adv. Mater. 28 (2016) 3115-3121. https://doi.org/10.1002/adma.201506025.

[281] R. Primavera, B.D. Kevadiya, G. Swaminathan, R.J. Wilson, A. De Pascale, P. Decuzzi, A.S. Thakor, Emerging Nano- and Micro-Technologies Used in the Treatment of Type-1 Diabetes, 1 (n.d.) 1-27.

[282] K.M. Prasanna Kumar, M. Chawla, A. Sanghvi, N.G. Sastry, C. Kotla, S. Phatak, S. Choudhari, Adherence, satisfaction, and experience with metformin $500 \mathrm{mg}$ prolonged release formulation in Indian patients with type 2 diabetes mellitus: A postmarketing observational study, Int. J. Gen. Med. 12 (2019) 147-159. https://doi.org/10.2147/IJGM.S179622.

[283] X.D.G. Xuan Jin, Dan Dan Zhu, Bo Zhi Chen, Mohammad Ashfaq, Insulin delivery systems combined with microneedle technology, Adv. Drug Deliv. Rev. 127 (2018) 119-137. https://doi.org/https://doi.org/10.1016/j.addr.2018.03.011.

[284] Z. Baghban Taraghdari, R. Imani, F. Mohabatpour, A Review on Bioengineering Approaches to Insulin Delivery: A Pharmaceutical and Engineering Perspective, Macromol. Biosci. 19 (2019) 1-21. https://doi.org/10.1002/mabi.201800458.

[285] World Health Organization WHO, Classification of Diabetes Mellitus 2019, Geneve, 2019. https://doi.org/CC BY-NC-SA 3.0 IGO.

[286] J. Yu, J. Wang, Y. Zhang, G. Chen, W. Mao, Y. Ye, Glucose-responsive insulin patch for the regulation of blood glucose in mice and minipigs, Nat. Biomed. Eng. 4 (2020) 499-506. https://doi.org/10.1038/s41551-019-0508-y.

[287] S. Chen, H. Matsumoto, Y. Moro-Oka, M. Tanaka, Y. Miyahara, T. Suganami, A. Matsumoto, Smart Microneedle Fabricated with Silk Fibroin Combined Semi-interpenetrating Network Hydrogel for Glucose-Responsive Insulin Delivery, ACS Biomater. Sci. Eng. 5 (2019) 57815789. https://doi.org/10.1021/acsbiomaterials.9b00532.

[288] S. Wang, Shiyi; Zhu, Mingmei; Zhao, Liang; Kuang, Dajiang; Kundu, Subhas C.; Lu, Insulinloaded silk fibroin microneedles as sustained release system, ACS Biomater. Sci. Eng. 5 (2019) 1887-1894. https://doi.org/10.1021/acsbiomaterials.9b00229.

[289] D. Liu, B. Yu, G. Jiang, W. Yu, Y. Zhang, B. Xu, Fabrication of composite microneedles integrated with insulin-loaded $\mathrm{CaCO} 3$ microparticles and PVP for transdermal delivery in diabetic rats, Mater. Sci. Eng. C. 90 (2018) 180-188. https://doi.org/10.1016/j.msec.2018.04.055.

[290] L. Li, G. Jiang, W. Yu, D. Liu, H. Chen, Y. Liu, Q. Huang, Z. Tong, J. Yao, X. Kong, A composite hydrogel system containing glucose-responsive nanocarriers for oral delivery of insulin, Mater. Sci. Eng. C. 69 (2016) 37-45. https://doi.org/10.1016/j.msec.2016.06.059.

[291] S. Kim, H. Yang, J. Eum, Y. Ma, S. Fakhraei Lahiji, H. Jung, Implantable powder-carrying microneedles for transdermal delivery of high-dose insulin with enhanced activity, Biomaterials. 
[292] K.-Y. Seong, M.-S. Seo, D.Y. Hwang, E.D. O'Cearbhaill, S. Sreenan, J.M. Karp, S.Y. Yang, A self-adherent, bullet-shaped microneedle patch for controlled transdermal delivery of insulin, J. Control. Release. 265 (2017) 48-56. https://doi.org/10.1016/j.jconrel.2017.03.041.

[293] B.Z. Chen, L.Q. Zhang, Y.Y. Xia, X.P. Zhang, X.D. Guo, A basal-bolus insulin regimen integrated microneedle patch for intraday postprandial glucose control, Sci. Adv. 6 (2020) 112. https://doi.org/10.1126/sciadv.aba7260.

[294] N.R. Jabir, S. Tabrez, G.M. Ashraf, S. Shakil, G.A. Damanhouri, M.A. Kamal, Nanotechnologybased approaches in anticancer research., Int. J. Nanomedicine. 7 (2012) 4391-4408. https://doi.org/10.2147/IJN.S33838.

[295] K.B. Sutradhar, M.L. Amin, Nanotechnology in Cancer Drug Delivery and Selective Targeting, ISRN Nanotechnol. 2014 (2014) 939378. https://doi.org/10.1155/2014/939378.

[296] Y. (Chezy) Barenholz, Doxil@ — The first FDA-approved nano-drug: Lessons learned, J. Control. $\quad$ Release. $\quad 160 \quad$ (2012) 117-134. https://doi.org/https://doi.org/10.1016/j.jconrel.2012.03.020.

[297] E. Miele, G.P. Spinelli, E. Miele, F. Tomao, S. Tomao, Albumin-bound formulation of paclitaxel (Abraxane ABI-007) in the treatment of breast cancer., Int. J. Nanomedicine. 4 (2009) 99-105. https://doi.org/10.2147/ijn.s3061.

[298] F.A.U. Rahman, S. Ali, M.W. Saif, Update on the role of nanoliposomal irinotecan in the treatment of metastatic pancreatic cancer., Therap. Adv. Gastroenterol. 10 (2017) 563-572. https://doi.org/10.1177/1756283X17705328.

[299] Y. Ma, S.E. Boese, Z. Luo, N. Nitin, H.S. Gill, Drug coated microneedles for minimally-invasive treatment of oral carcinomas: development and in vitro evaluation., Biomed. Microdevices. 17 (2015) 44. https://doi.org/10.1007/s10544-015-9944-y.

[300] S.R. Vučen, G. Vuleta, A.M. Crean, A.C. Moore, N. Ignjatovië, D. Uskokovië, Improved percutaneous delivery of ketoprofen using combined application of nanocarriers and silicon microneedles, J. Pharm. Pharmacol. 65 (2013) 1451-1462. https://doi.org/10.1111/jphp.12118.

[301] C. Wang, Y. Ye, G.M. Hochu, H. Sadeghifar, Z. Gu, Enhanced Cancer Immunotherapy by Microneedle Patch-Assisted Delivery of Anti-PD1 Antibody, Nano Lett. 16 (2016) 2334-2340. https://doi.org/10.1021/acs.nanolett.5b05030.

[302] Z. Jaulim, N. Salmon, C. Fuller, Fungal skin infections: current approaches to management, Prescriber. 26 (2015) 31-35. https://doi.org/10.1002/psb.1394.

[303] A.H. Sabri, J. Ogilvie, K. Abdulhamid, V. Shpadaruk, J. McKenna, J. Segal, D.J. Scurr, M. Marlow, Expanding the applications of microneedles in dermatology, Eur. J. Pharm. Biopharm. 140 (2019) 121-140. https://doi.org/10.1016/j.ejpb.2019.05.001.

[304] Z. Zhao, Y. Chen, Y. Shi, Microneedles: A potential strategy in transdermal delivery and application in the management of psoriasis, RSC Adv. 10 (2020) 14040-14049. https://doi.org/10.1039/d0ra00735h.

[305] B. Pamornpathomkul, T. Ngawhirunpat, I.A. Tekko, L. Vora, H.O. McCarthy, R.F. Donnelly, Dissolving polymeric microneedle arrays for enhanced site-specific acyclovir delivery, Eur. J. Pharm. Sci. 121 (2018) 200-209. https://doi.org/10.1016/j.ejps.2018.05.009.

[306] J.A. ODaly, Psoriasis, a systemic disease beyond the skin, as evidenced by psoriatic arthritis and many comorbities - clinical cemission with a leishmania amastigotes vaccine, a serendipity finding, Psoriasis - A Syst. Dis. 18 (2012) 1-56. https://doi.org/10.5772/25789.

[307] N. Parnami, T. Garg, G. Rath, A.K. Goyal, Development and characterization of nanocarriers for topical treatment of psoriasis by using combination therapy, Artif. Cells, Nanomedicine Biotechnol. 42 (2014) 4-6-412. https://doi.org/10.3109/21691401.2013.837474.

[308] G. Raho, D.M. Koleva, L. Garattini, L. Naldi, The burden of moderate to severe psoriasis: An overview, Pharmacoeconomics. 30 (2012) 1005-1013. https://doi.org/10.2165/11591580000000000-00000.

[309] W.B. Kim, D. Jerome, J. Yeung, Diagnosis and management of psoriasis, Can. Fam. Physician. 63 (2017) 278-285.

[310] A.E. Bello, E.L. Perkins, R. Jay, P. Efthimiou, Recommendations for optimizing methotrexate treatment for patients with rheumatoid arthritis, (2017). https://doi.org/10.2147/OARRR.S131668. 
[311] H.R. Jeong, J.Y. Kim, S.N. Kim, J.H. Park, Local dermal delivery of cyclosporin A, a hydrophobic and high molecular weight drug, using dissolving microneedles, Eur. J. Pharm. Biopharm. 127 (2018) 237-243. https://doi.org/10.1016/j.ejpb.2018.02.014.

[312] R.R.S. Thakur, I.A. Tekko, F. Al-Shammari, A.A. Ali, H. McCarthy, R.F. Donnelly, Rapidly dissolving polymeric microneedles for minimally invasive intraocular drug delivery, Drug Deliv. Transl. Res. (2016). https://doi.org/10.1007/s13346-016-0332-9.

[313] D. Mishra, S. Gade, T. Raghu, R. Singh, Ocular Disorders and Associated Drug Development Challenges: The Current Scenario, EC Ophthalmol. 11 (2020) 92-101.

[314] G. Yiu, S.H. Chung, I.N. Mollhoff, U.T. Nguyen, S.M. Thomasy, J. Yoo, D. Taraborelli, G. Noronha, Suprachoroidal and Subretinal Injections of AAV Using Transscleral Microneedles for Retinal Gene Delivery in Nonhuman Primates, Mol. Ther. - Methods Clin. Dev. 16 (2020) 179-191. https://doi.org/10.1016/j.omtm.2020.01.002.

[315] R.R.S. Thakur, S.J. Fallows, H.L. McMillan, R.F. Donnelly, D.S. Jones, Microneedle-mediated intrascleral delivery of in situ forming thermoresponsive implants for sustained ocular drug delivery, J. Pharm. Pharmacol. 66 (2014) 584-595. https://doi.org/10.1111/jphp.12152.

[316] citations-20200823T130849, (n.d.).

[317] J.H. Jung, S. Park, J.J. Chae, M.R. Prausnitz, Collagenase injection into the suprachoroidal space of the eye to expand drug delivery coverage and increase posterior drug targeting, Exp. Eye Res. 189 (2019) 107824. https://doi.org/https://doi.org/10.1016/j.exer.2019.107824.

[318] J.H. Jung, P. Desit, M.R. Prausnitz, Targeted Drug Delivery in the Suprachoroidal Space by Swollen Hydrogel Pushing., Invest. Ophthalmol. Vis. Sci. 59 (2018) 2069-2079. https://doi.org/10.1167/iovs.17-23758.

[319] M. Amer, R.K. Chen, Self-Adhesive Microneedles with Interlocking Features for Sustained Ocular Drug Delivery, Macromol. Biosci. $20 \quad$ (2020) 2000089. https://doi.org/10.1002/mabi.202000089.

[320] K. Saraswathy, G. Agarwal, A. Srivastava, Hyaluronic acid microneedles-laden collagen cryogel plugs for ocular drug delivery, J. Appl. Polym. Sci. (2020) 1-14. https://doi.org/10.1002/app.49285.

[321] G. Roy, R.D. Galigama, V.S. Thorat, L.S. Mallela, S. Roy, P. Garg, V.V.K. Venuganti, Amphotericin B containing microneedle ocular patch for effective treatment of fungal keratitis, $\begin{array}{lllll}\text { Int. } & \text { J. } & \text { Pharm. } & 572 & \text { (2019) }\end{array}$ https://doi.org/https://doi.org/10.1016/j.ijpharm.2019.118808.

[322] R.F. Donnelly, How can microneedles overcome challenges facing transdermal drug delivery?, Ther. Deliv. 8 (2017) 725-728. https://doi.org/10.4155/tde-2017-0028.

[323] R.F. Donnelly, Clinical Translation and Industrial Development of Microneedle-based Products, Microneedles Drug Vaccine Deliv. Patient Monit. (2018) 307-322. https://doi.org/doi:10.1002/9781119305101.ch11.

[324] S. Hua, M.B.C. de Matos, J.M. Metselaar, G. Storm, Current trends and challenges in the clinical translation of nanoparticulate nanomedicines: Pathways for translational development and commercialization, Front. Pharmacol. 9 (2018) 1-14. https://doi.org/10.3389/fphar.2018.00790.

[325] M. Kirkby, A.R.J. Hutton, R.F. Donnelly, Microneedle Mediated Transdermal Delivery of Protein, Peptide and Antibody Based Therapeutics: Current Status and Future Considerations, Pharm. Res. 37 (2020) 117. https://doi.org/10.1007/s11095-020-02844-6.

[326] L.-P. Wu, D. Wang, Z. Li, Grand challenges in nanomedicine, Mater. Sci. Eng. C. 106 (2020) 110302. https://doi.org/https://doi.org/10.1016/j.msec.2019.110302.

[327] A.J. Courtenay, E. McAlister, M.T.C. McCrudden, L. Vora, L. Steiner, G. Levin, E. LevyNissenbaum, N. Shterman, M.-C. Kearney, H.O. McCarthy, R.F. Donnelly, Hydrogel-forming microneedle arrays as a therapeutic option for transdermal esketamine delivery, J. Control. Release. 322 (2020) 177-186. https://doi.org/https://doi.org/10.1016/j.jconrel.2020.03.026.

[328] H.E. Gendelman, J.E. McMillan, A.N. Bade, B. Edagwa, B.D. Kevadiya, The Promise of LongActing Antiretroviral Therapies: From Need to Manufacture, Trends Microbiol. 27 (2019) 593606. https://doi.org/10.1016/j.tim.2019.02.009.

[329] D. Guidance, Regulatory Considerations for Microneedling Devices Draft Guidance for Industry and, (2017) 1-12.

[330] Top 10 Emerging Technologies of 2020, 2020 World Econ. Forum. 317 (2020) 28-39. 
https://doi.org/10.1038/scientificamerican1217-28.

[331] Zosano Pharma Receives Complete Response Letter from FDA for Qtrypta, (n.d.). https://www.drugs.com/nda/qtrypta_201021.html (accessed November 22, 2020).

[332] PATH, The PATH Center of Excellence for Microarray Patch Technology |PATH, (2019).

3061 